\title{
SOLA-DM \\ A Numerical Solution Algorithm for Transient Three-Dimensional Flows
}

\author{
T. L. Wilson \\ B. D. Nichols \\ C. W. Hirt" \\ L. R. Stein**
}

DISCLAIMER

This report was prepared as an account of work sponsored by an agency of the United States Government. Neither the United States Government nor any agency thereof, nor any of their employees, makes any warranty, express or implied, or assumes any legal liability or responsibility for the accuracy, completeness, or usefulness of any information, apparatus, product, or process disclosed, or represents that its use would not infringe privately owned rights. Reference herein to aiy specific commercial product, process, or service by trade name, trademark, manufacturer, or otherwise does not necessarily constitute or imply its endorsement, recommendation, or favoring by the United States Government or any agency thereof. The views and opinions of authors expressed herein do not necessarily state or reffect those of the United States Government or any agency thereof. 


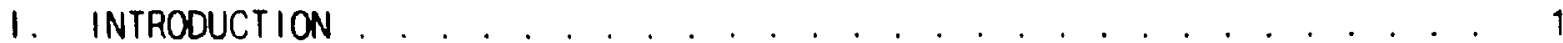

11. THE FLUID DYNAMICS SOLUTION ALGORITHM (SOLA) . . . . . . . . . . 3

A. Equations of Motion ................ . . . 3

1. Nonrotating Coordinate System . . . . . . . . . . . . . . . 3

2. Rotating Coordinate System . . . . . . . . . . . . . . . . 4

B. Finite-Difference Considerations ........... . 4

C. Outline of Finite-Difference Solution Method . . . . . . . . . . 7

D. Momentum Equation Approximations . . . . . . . . . . . . . . . . 7

E. Cont inuity Equation Approximation . . . . . . . . . . . . . . . 11

111. THE PARTICLE DYNAMICS SOLUTION ALGORITHM . . . . . . . . . . . . . 14

A. Equations of Motion . . . . . . . . . . . . . . . . . . 15

B. Fluid-Dynamic Drag . . . . . . . . . . . . . . . 16

1. Stokes Drag . . . . . . . . . . . . . . . . . . . . . 16

2. Form Drag . . . . . . . . . . . 17

C. Particle Diffusion . . . . . . . . . . . . . 18

D. Out line of Numerical Solution Method. . . . . . . . . . . . . 19

E. Approximate Particle Motion Equations . . . . . . . . . . . . 20

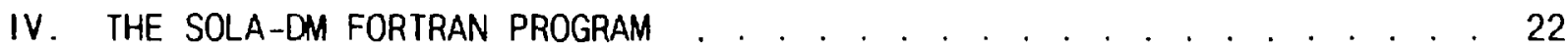

A. Code Structure (Subroutine List) . . . . . . . . . . . . . . . . 22

B. Single Indexing Scheme . . . . . . . . . . . . . . . . . . . 29

C. Automat ic Mesh Generator . . . . . . . . . . . . . . . . 31

D. Boundary Cond itions . . . . . . . . . . . . . . . . . . . . . . 33

1. Mesh Boundar ies . . . . . . . . . . . . . . . . . . . . . . 33

2. Internal Obstacle Boundaries . . . . . . . . . . . . 37

E. Numerical Stability Considerations . . . . . . . . . . . . . . 38

F. Graphics . . . . . . . . . . . . . . . . . . . . . . . 39

V. RUNNING A PROBLEM .................. . . 42

A. Preparing an Input Data File... . . . . . . . . . . . . . 42

1. NAMELIST Convention ........... . . . 42

2. Physical/Numerical/Control Data (NAMELIST/XPUT/) . . . . 43

3. Mesh Generation Data (NAMELIST/MESHGN/) . . . . . . . . . 45

4. Graphic Output Data (NAMELIST/GRAFIC/) . . . . . . . . . . 46

5. Particle Input Data (NAMELIST/PARTS/) . . . . . . . . . 48 
B. Code Modifications . . . . . . . . . . . . . . . . . 50

1. Special Boundary Conditions . . . . . . . . . . . . . . . . 50

2. Modifying the IBETA Array . . . . . . . . . . . . . . . . . 50

3. Global Cont inuity . . . . . . . . . . . . . . . . . . 50

4. Ar ray Dimens ion Requi rements . . . . . . . . . . . . 51

C. Output . . . . . . . . . . . . . . 52

D. Restarting a Problem . . . . . . . . . . . 52

E. Sample Test Problem . . . . . . . . . . . . . . . . 53

REFERENCES . . . . . . . . . . . . . . . . . . . . 56

APPENDIX A. SYSTEM SUBROUTINE CALLS IN SOLA-DM . . . . . . . . . . . . . 57

APPENDIX B. INPUT AND OUTPUT FILES FOR SAMPLE PROBLEM . . . . . . . . . . 59 
SOLA-DM

A NUMERICAL SOLUTION ALGORITHM

FOR TRANSIENT THREE-DIMENSIONAL FLOWS

by

T. L. Wilson, B. D. Nichols, C. W. Hirt, and L. R. Stein

\begin{abstract}
SOLA-OM is a three-dimensional time-explicit, finite-difference, Eulerian, fluid-dynamics computer code for solving the time-dependent incompressible Navier-Stokes equations. The solut ion algor ithm (SOLA) evolved from the marker-and-cell (MAC) method, and the code is highly vectorized for efficient performance on a Cray computer.

The computational domain is discretized by a mesh of parallelepiped cells in either cartesian or cylindrical geometry. The primary hydrodynamic variables for approximating the solution of the momentum equations are cell-face-centered velocity components and cell-centered pressures. Spatial accuracy is selected by the user to be first or second order; the time differencing is first-order accurate.

The incompressibility condition results in an elliptic equation for pressure that is solved by a conjugate gradient method.

Boundary conditions of five general types may be chosen: freeslip, no-slip, continuative, periodic, and specified pressure. In addition, internal mesh specifications to model obstacles and walls are provided. SOLA-DM also solves the equations for discrete particle dynamics, permitting the transport of marker particles or other solid particles through the fluid to be modeled.
\end{abstract}

\title{
I. INTRODUCTION
}

This report describes a computer code for calculating the dynamics of an incompressible fluid in three space dimensions. The program, SOLA-3D, evolved 
from the well-known marker-and-cell (MAC) finite-difference technique, 1,2 which uses pressure and velocity as the primary dependent variables. Highly simplified two-dimensional solution algorithms (SOLA and SOLA-SURF) have been publicly available for several years. 3 These programs were designed for use by persons with little or no experience in numerical fluid dynamics. In addition to serving as instructional tools, they have been used for numerous applications and have served as foundations for the development of a variety of other programs. SOLA-3D is a three-dimensional extension of the SOLA and SOLA-SURF codes with several additional features. A variable mesh capability has been used to help increase numerical accuracy (resolution) for a given amount of computer storage. Single indexing is used to increase computational speed, but code readability has been maintained by using a simple acronym scheme. Either cartesian $(x, y, z)$ or cylindrical $(r, \theta, z)$ coordinate systems can be selected through input data. Code options allow the user to model internal obstacles, walls, and the transport of discrete particles. A version of the code described here, SOLA-DM, has been developed to model fluid flow and dust particle motion associated with personal dust monitors used to sample air quality in mines.

The basic solution algorithm used in SOLA-DM is described in Sec. 11 . Special consideration is given to the finite-difference approximations used to maintain formal accuracy in both uniform and nonuniform meshes. The basic algorithm is first-order accurate in space and time, but a second-order-accurate option is available.

The discrete particle dynamics option is discussed in Sec. 111. The particles represent small masses that move under the combined influences of gravity, buoyancy, and fluid drag forces. In this version of SOLA-DM, the particles do not influence the fluid dynamics; therefore, completely coupled dynamic calculations are not possible.

The programming details are covered in Sec. IV and include explanations of the subroutines, single indexing schemes, mesh generation, boundary conditions, numerical stability, and computer graphic routines.

Instructions for running a problem are given in Sec. $V$. This includes directions for preparing an input file, modifying the code, and restarting a problem. A sample problem is given to illustrate the process. 
11. THE FLUID DYNAMICS SOLUTION ALGORITHM (SOLA)

\section{A. Equations of Motion}

1. Nonrotating Coordinate System. The differential equations to be solved are written in terms of Cartesian coordinates ( $x, y, z)$. For cylindrical coordinates $(r, \theta, z)$, the $x$ coordinate is interpreted as the radial direction: the $y$ coordinate is transformed to the curvilinear azimuthal coordinate, $r \theta$; and $z$ is the axial coordinats. In addition. several terms must be added to the cartesian equations of motion for cylindrical geometry. In the following, these terms are included with a coefficient, $\xi$, such that $\xi=0$ corresponds to cartesian geometry and $\xi=1$ corresponds to cylindrical geometry. The SOLA-DM code uses the input parameter CYL as the value of $\xi$.

The mass continuity equation for an incompressible fluid is

$$
\frac{\partial u}{\partial x}+\frac{\partial v}{\partial y}+\frac{\partial w}{\partial z}+\xi \frac{u}{x}=0
$$

The velocity components $(u, v, w)$ are in the coordinate directions $(x, y, z)$ or $(r, \theta, z)$.

The equations of motion for the fluid velocity components in the three coordinate directions $(u, v, w)$ are the Navier-Stokes equations:

$$
\begin{aligned}
& \frac{\partial u}{\partial t}+u \frac{\partial u}{\partial x}+v \frac{\partial u}{\partial y}+w \frac{\partial u}{\partial z}-\xi \frac{v^{2}}{x}=-\frac{\partial P}{\partial x}+g_{x}+f_{x}, \\
& \frac{\partial v}{\partial t}+u \frac{\partial v}{\partial x}+v \frac{\partial v}{\partial y}+w \frac{\partial v}{\partial z}+\xi \frac{u v}{x}=-\frac{\partial P}{\partial y}+g_{y}+f_{y}, \text { and } \\
& \frac{\partial w}{\partial t}+u \frac{\partial w}{\partial x}+v \frac{\partial w}{\partial y}+w \frac{\partial w}{\partial z}=-\frac{\partial P}{\partial z}+g_{z}+f_{z} .
\end{aligned}
$$

In these equations. $P$ is the fluid pressure divided by the density, $\left(g_{x}, g_{y}, g_{z}\right)$ are body accelerations, and $\left(f_{x}, f_{y}, f_{z}\right)$ are viscous accelerations. For a constant kinematic viscosity $v$, the viscous accelerations are 


$$
\begin{aligned}
& f_{x}=v\left[\frac{\partial^{2} u}{\partial x^{2}}+\frac{\partial^{2} u}{\partial y^{2}}+\frac{\partial^{2} u}{\partial z^{2}}+\xi\left(\frac{1}{x} \frac{\partial u}{\partial x}-\frac{u}{x^{2}}-\frac{2}{x} \frac{\partial v}{\partial y}\right)\right], \\
& f_{y}=v\left[\frac{\partial^{2} v}{\partial x^{2}}+\frac{\partial^{2} v}{\partial y^{2}}+\frac{\partial^{2} v}{\partial z^{2}}+\xi\left(\frac{1}{x} \frac{\partial v}{\partial x}-\frac{v}{x^{2}}+\frac{2}{x} \frac{\partial u}{\partial y}\right)\right], \text { and } \\
& f_{z}=v\left[\frac{\partial^{2} w}{\partial x^{2}}+\frac{\partial^{2} w}{\partial y^{2}}+\frac{\partial^{2} w}{\partial z^{2}}+\frac{\xi}{x} \frac{\partial w}{\partial x}\right] .
\end{aligned}
$$

2. Rotating Coordinate System. In certain applications, it is desirable to use a rotating cylindrical coordinate system. A good example would be some sort of rotating machinery in which mesh cells are blocked out to represent rigid internal parts. By transforming to the coordinate system rotating with the device, it is possible to eliminate the need for moving internal obstacles through a mesh. Let the constant angular velocity be denoted by $\Omega$ radians per unit time. A rotating coordinate system is not an inertial frame of reference, so the equations of motion [Eqs. (2)] must be modified. In particular, we must add $\left(2 \Omega v+r \Omega^{2}\right)$ to the right side of the $u$ equation and $-2 \Omega u$ to the right side of the $v$ equation. No changes are required in the $w$ equation, the continuity equation, or the viscous accelerations.

\section{B. Finite-Difference Considerations}

The finite-difference mesh used for solving the above equations numerically consists of parallelepiped cells of width $\delta x_{i}$, Jepth $\delta y_{j}$, and height $\delta z_{k}$. The active mesh region consists of IBAR cells in the $x$ direction labeled with the index $i$, JBAR cells in the $y$ direction labeled with the index $j$, and KBAR cells in the $z$ direction labeled with the index $k$. In terms of the cell labeling nomenclature shown in Fig. $1, \quad I B A R=I M 1-1, J B A R=J M 1-1$, and $K B A R=K M 1-1$. This region is surrounded by a layer of fictitious or boundary cells used to set mesh boundary conditions. Thus, there are usually $($ IBAR +2$) *(J B A R+2)$ * $($ KBAR +2$)$ total relis in a complete mesh. Figure 2 shows the placement of boundary cells (dashed lines) for a slice of the mesh normal to the $z$ direction (a " $K$ " plane). However, when periodic boundary conditions are used, one additional layer of fictitious cells is used in each direction having periodicity. 


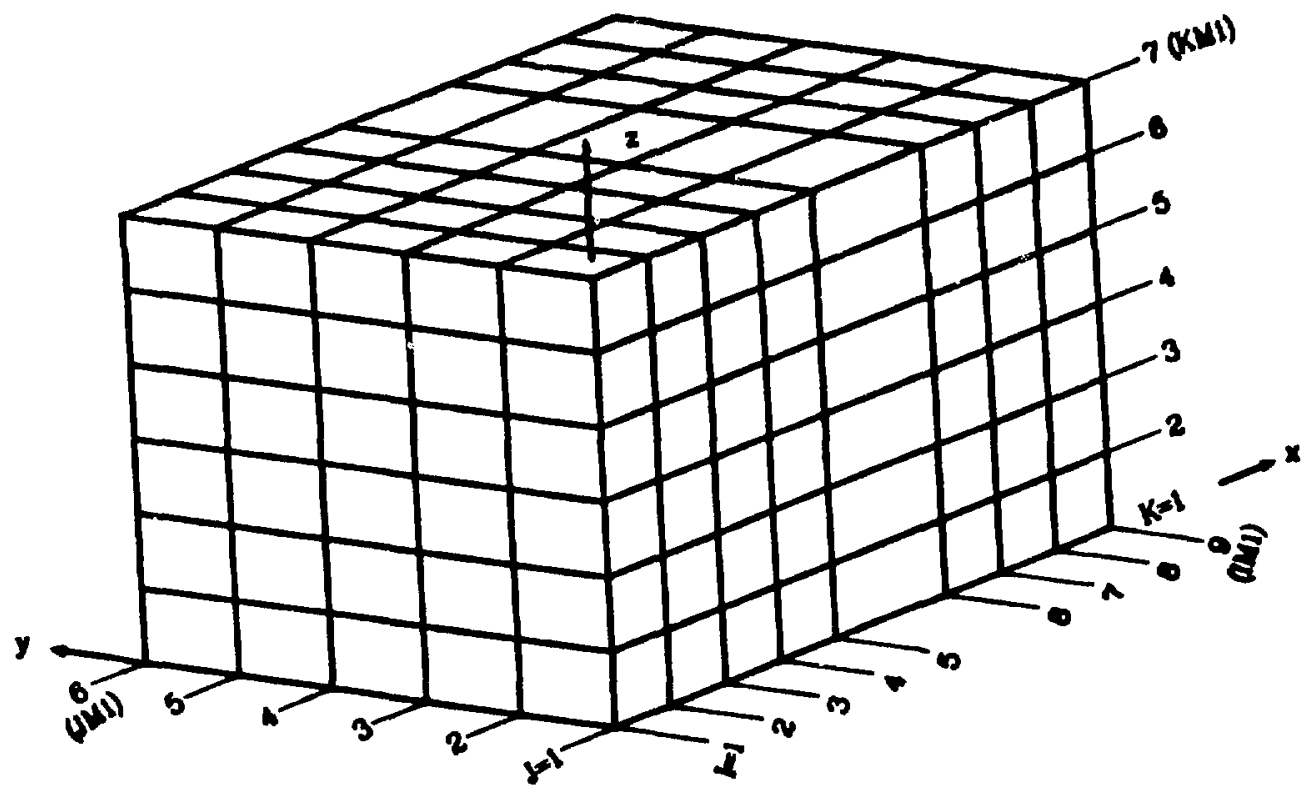

Fig. 1.

Three-dimensional mesh of computational cells with grid index labeling convention.

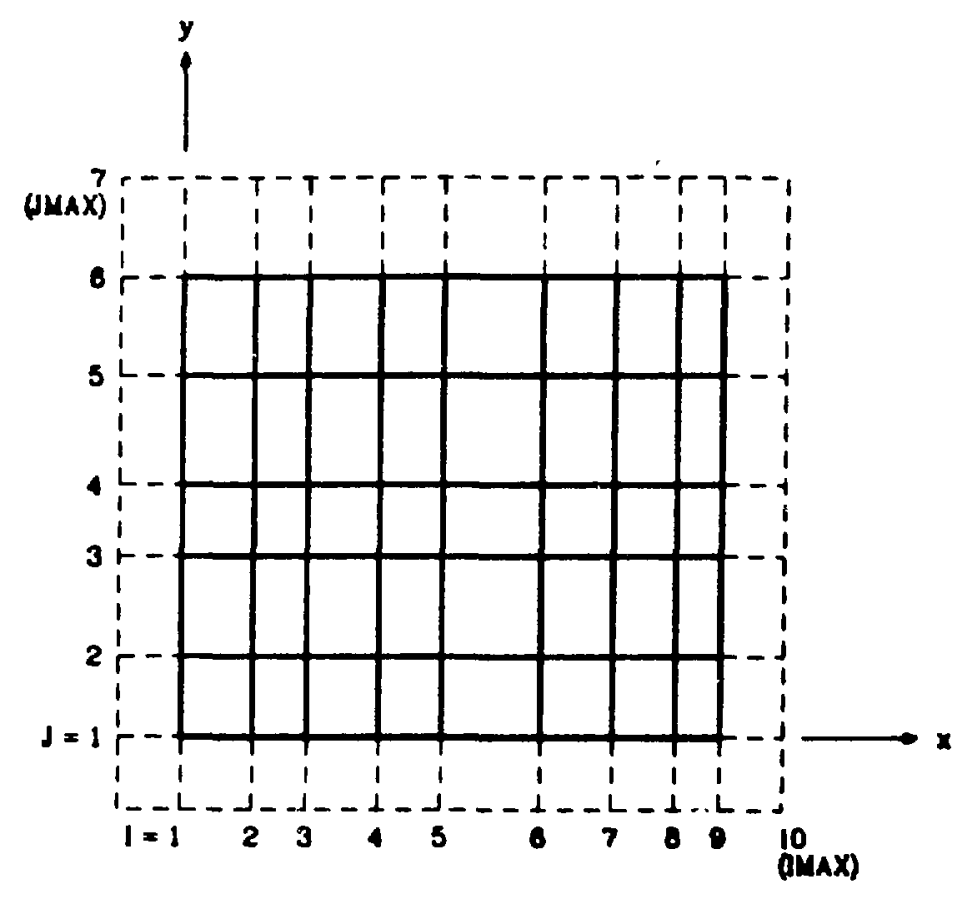

Fig. 2 .

Two-dimensional mesh slice in the $x-y$ plane. Boundary cells are drawn with dashed lines. 
This must be remembered only when setting dimension statements. The mesh generator (described in Sec. IV.C) will initialize the necessary number of boundary cells needed to satisfy the boundary conditions automatically.

A typical mesh cell [identified by logical indices $(i, j, k)$ ] is shown in Fig. 3. The cell-centered pressure is written as $p^{n} i, j, k$ where the superscript $n$ refers to the $n$th time-step value. Fluid velocity components are located at the middle of cell sides (faces). The u velocity with indices ( $i, j, k$ ), which is denoted by $u_{i, j, k}$, is located on the cell face between cells $(i, j, k)$ and $(i+1, j, k)$. The velocity components $v_{i, j, k}$ and $w_{i}, j, k$ are defined similarly for the $y$ and $z$ directions. This convention means that the velocity components with indices $(i, j, k)$ are identified with the three cell faces having positive outward normals on cell $(i, j, k)$. Thus,

$$
\begin{aligned}
& P_{i, j, k}^{n}=\text { pressure at center of cell }(i, j, k) \text { at time level } n, \\
& u_{i, j, k}^{n}=\begin{array}{l}
x \text {-direction velocity at middle of positive } x \text { cell face at time } \\
\text { level } n,
\end{array}
\end{aligned}
$$

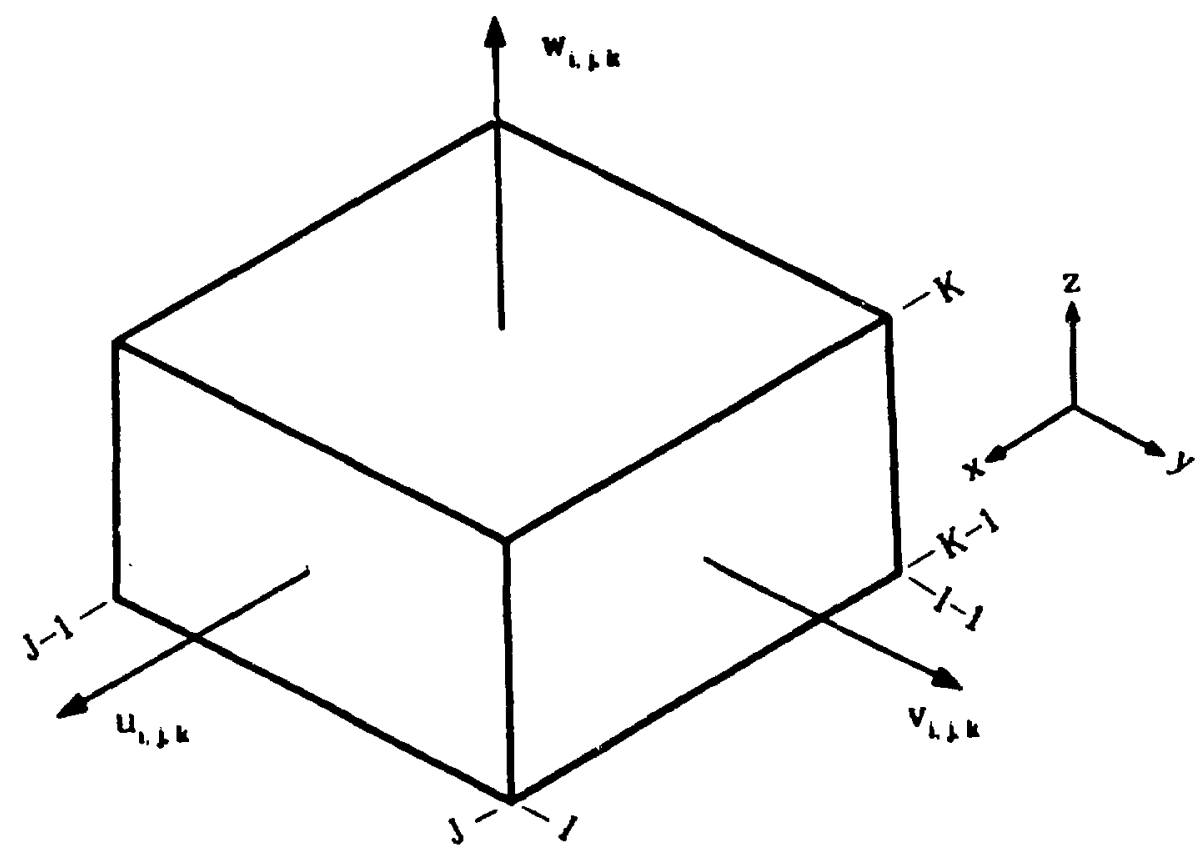

Fig. 3 .

Velocity components identified with mesh cell $(i, j, k)$. (The positive values indicated for $u, v$, and $w$ are drawn for clarity of illustration.) 


$$
\begin{aligned}
& v_{i, j, k}^{n}= y \text {-direction velocity at middle of positive y cell face at time } \\
& \text { level } n, \text { and }
\end{aligned}
$$

\section{Outl ine of Finite-Difference Solution Method}

The basic procedure for advancing a solution through one increment in time, $\delta t$, consists of two steps.

(1) Explicit approximations of the momentum equations [Eqs. (2)] are used to compute the first guess for new time-level velocities using the previous time-level values for all advective, pressure, and other accelerations.

(2) To satisfy the continuity equation [Eq. (1)], pressures are adjusted iteratively in each cell, and the velocity changes induced by each pressure change are added to the velocities computed in step (1). An iteration is needed because the change in pressure needed in one cell to satisfy Eq. (1) will upset the balance in the six adjacent cells.

Repeating these steps will advance a solution through any desired time interval. At each step, suitable boundary conditions must be imposed at all mesh surfaces. These steps and boundary conditions are discussed in detail below.

\section{Momentum Equation Approximations}

Using the index convention defined in Sec. II.B, a generic form for the finite-difference approximations of Eqs. (2) is

$$
\begin{aligned}
u_{i, j, k}^{n+1}= & u_{i, j, k}^{n}+\delta t\left[-\left(P_{i+1, j, k}^{n+1}-P_{i, j, k}^{n+1}\right) / \delta x c_{i}\right. \\
& \left.+g_{x}-F U X-F U Y-F U Z+V I S X+R O T X\right],
\end{aligned}
$$




$$
\begin{aligned}
v_{i, j, k}^{n+1} & =v_{i, j, k}^{n}+\delta t\left[-\left(P_{i, j+1, k}^{n+1}-P_{i, j, k}^{n+1}\right) / \delta y c_{j}\right. \\
& \left.+g_{y}-F V X-F V Y-F V Z+V I S Y+R O T Y\right] \text {, and } \\
w_{i, j, k}^{n+1}= & w_{i, j, k}^{n}+\delta t\left[-\left(P_{i, j, k+1}^{n+1}-P_{i, j, k}^{n+1}\right) / \delta z c_{k}\right. \\
& \left.+g_{y}-F W X-F W Y-F W Z+V I S Z\right],
\end{aligned}
$$

where, for example, $\delta x_{i}=\left(\delta x_{i}+\delta x_{i+1}\right) / 2$ is the distance between the centers of cells $(i, j, k)$ and $(i+1, j, k)$.

The advective, viscous, and rotational acceleration terms have an obvious meaning; for example, FUX means the advective flux of $u$ in the $x$ direction. VISX are the $x$-component viscous accelerations, and ROTX are the $x$-component rotational accelerations associated with a rotating coordinate system. These ternis are evaluated using old time-level $(n)$ values for velocities. Because the pressures at time level $n+1$ are unknown at the beginning of the cycle, these equations canriot be used directly to evaluate the $n+1$ level velocities but must be combined with the continuity equation as described below. Therefore, in the first step of a solution, the $p^{n+1}$ values in these equations are replaced by $p^{n}$ values to get a first guess for the new velocities.

In the basic solution procedure, the skecific approximations chosen for the various acceleration terms in Eqs. (4) are relatively unimportant provided they lead to a numerically stable algorithm. However, special care must be taken when making approximations in a mesh with nonuniform cell sizes. This problem has been discussed elsewher $e^{4}$ but is noted here because it often has been overlooked. Consider the approximation procedure used in the original MAC method ${ }^{1,2}$ for cartesian coordinates. In the MAC scheme, the momentum and continuity equations [Eqs. (1) and (2)] first were combined so that the advective flux terims could be written in a divergence form (that is, ק.uu instead of $\underline{u} \nabla \underline{u}$ ). Thus, Fux would be $\partial u^{2} / \partial x$ rather than uau/dx. The divergence form was preferred in MAC because it provided a simple way to insure conservation of momentum in the difference approximations. This may be seen by considering an $x-y$ cross section of the control volume used for $u_{i}$ as indicated by dashed lines in Fig. 4. With the divergence form, Gauss' theorem may be used to convert the integrated value of 


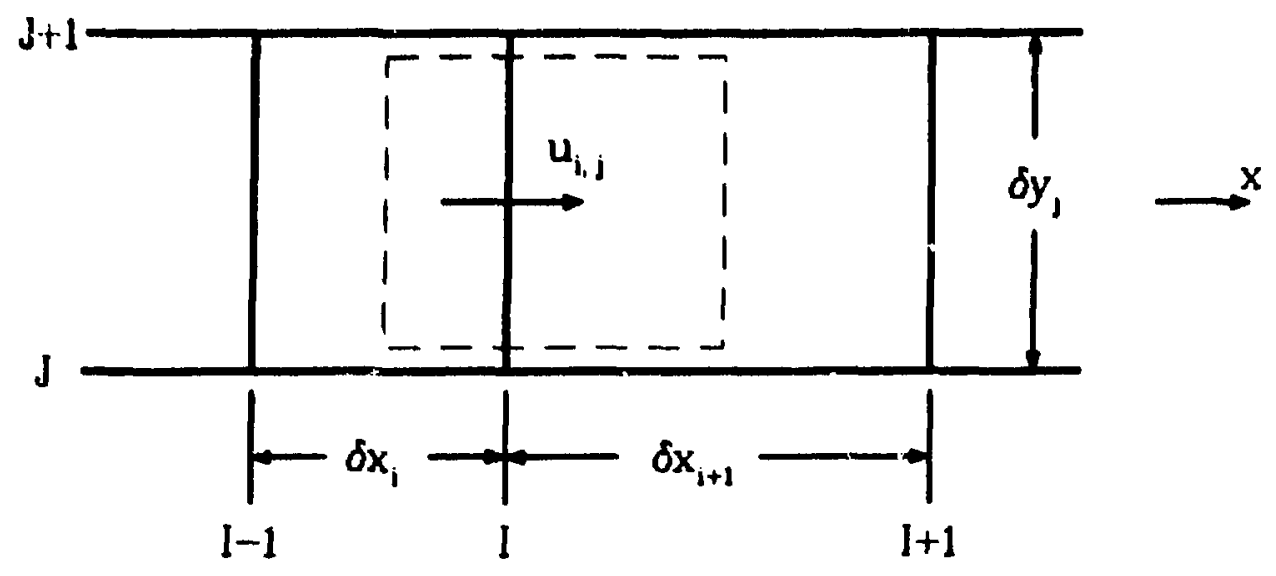

Fig. 4.

Control volume (dashed rectangle) used for constructing a finite-difference approximation for the $u$ momenturin equation on the positive $x$ face of cell $(i, j, k)$.

Fux over the control volume to boundary fluxes at its sides. The flux leaving one control volume then will be gained by the adjacent one, and conservatici: during advection is ensured.

This idea worked well in the original MAC scheme, which was developed for use in uniform meshes. Unfortunately, in nonuniform meshes, conservation does not automatically imply accuracy. To see this, suppose an upstream or donorcell difference approximation is used for FUX $=\partial u^{2} / \partial x$, which provides a conditionally stable algorithm. The donor-cell approximation is

$$
F U X=\left[u_{R}\left\langle u_{R}\right\rangle-u_{L}\left\langle u_{L}\right\rangle\right]^{/ \delta x c_{i}},
$$

where

$$
u_{R}=\left(u_{i+1, j, k}+u_{i, j, k}\right) / 2
$$

and

$$
\left\langle u_{R}\right\rangle=\left\{\begin{array}{ll}
u_{i, j, k}, & \text { if } u_{i, j, k} \geq 0 \\
u_{i+1, j, k}, & \text { if } u_{i, j, k}<0
\end{array}\right\} .
$$


To check the accuracy of Eq. (5), we expanded it in a Taylor series about the $x$ position where FUX is evaluated (assuming the $u$ velocities are positive):

$$
F U X=\frac{1}{2} \frac{3 \delta x_{i}+\delta x_{i+1}}{\delta x_{i}+\delta x_{i+1}} \frac{\partial u^{2}}{\partial x}+O(\delta x) .
$$

Clearly, the zeroth-order term is incorrect unless the cell widths are equal $\left(\delta x_{i}=\delta x_{i+1}\right)$. In other words, the variable mesh reduces the order of the conservative difference approximation by one, which in this case leads to an incorrect zeroth-order result. If a centered rather than a donor-cell approximation had been used, the result would have been first-order accurate, not second order as it is in a uniform mesh.

It does not follow from the above analysis that variable meshes are necessarily less accurate in practice because they do allow firier zoning to be used in regions where flow variables vary rapidly. Nevertheless, variable meshes! must be used with considerable care. It is best to use gradual variations in cell sizes to minimize the reduction in approximation order. It also is worthwhile to look for other approximations that do not lose their formal accuracy. when applied to variable,meshes. In this regard, it should be noted that the conservative forms of the advective terms lose accuracy because the control volumes are not centered about the positions where the variables to be updated are located. The shifted control volumes lead directly to the reduction in accuracy computed in Eq. (6).

For the SOLA series of codes, a modified donor-cell approximation has been developed that retains its accuracy in a variable mesh. This method approximates advective fluxes in the nonconservative form $\underline{u} \bullet \underline{\nabla}$, which is necessary because of the inherent difficulty with conservative approximations noted above. In the new approximation, it also is possible to combine the donor-cell and centered-difference approximations into a single expression with a parameter, $\alpha$, that controls the relative amount of each one. The general form of this approximation for FUX $=u \partial u / \partial x$ is

$$
F U X=\left(u_{C} / \delta x_{\alpha}\right)\left[\delta x_{i+1} D U L+\delta x_{i} D U R+\alpha \operatorname{sgn}\left(u_{C}\right)\left(\delta x_{i+1} D U L-\delta x_{i} D U R\right)\right] .
$$


where $u_{C}=u_{i, j, k}$ is the value of $u$ at the location where FUX is evaluated and

$$
\begin{aligned}
& \text { DUL }=\left(u_{i, j}, k-u_{i}-1, j, k\right) / \delta x_{i}, \\
& \text { DUR }=\left(u_{i+1}, j, k-u_{i, j, k}\right) / \delta x_{i}+1, \text { and } \\
& \delta x_{\alpha}=\delta x_{i+1}+\delta x_{i}+\alpha \operatorname{sgn}\left(u_{c}\right)\left(\delta x_{i+1}-\delta x_{i}\right) .
\end{aligned}
$$

The expression $\operatorname{sgn}\left(u_{C}\right)$ means the sign of $u_{C}$. When $\alpha=0$, this approximation reduces to a second-order-accurate, centered-difference approximation. When $\alpha=$ 1, the first-order, donor-cell approximation is recovered. In either case, there is no loss of formal accuracy in a variable mesh.

The basic idea underlying Eq. (7) is to weight the upstream derivative of the quantity being fluxed more than the downstream value. The weighting factors are $(1+\alpha)$ and $(1-\alpha)$ for the upstream and downstream derivatives, respectively. These derivatives also are weighted by cell size in such a way that the order of approximation is maintained in a variable mesh. This type of approximation scheme is used in SOLA-UM for all advective flux terms appearing in

Eq. (4). All other acceleration terms are approximated by standard centered differences.

\section{E. Continuity Equation Approximation}

Velocities computed from Eq. (4) must satisfy the following discretized approximation to Eq. (1), the continuity equation.

$$
\begin{aligned}
& \left(u_{i, j, k}-u_{i-1, j, k}\right) / \delta x_{i}+\left(v_{i, j, k}-v_{i, j-1, k}\right) / \delta y_{j} \\
& +\left(w_{i, j, k}-w_{i, j, k-1}\right) / \delta z_{k}+1 / 2 C Y L\left(u_{i, j, k}+u_{i-1, j, k}\right) / X C_{i}=0,
\end{aligned}
$$

where $X_{C}$ is the $x$ location of the center of cell $(i, j, k)$.

For velocities to satisfy Eq. (8), it is necessary to adjust the pressures, and hence the velocities, in each computational cell occupied by fluid. This is done by the following iterative process described in Ref. 5. Combining the continuity equations, 


$$
\nabla \cdot \underline{u}^{n+1}=0
$$

together with the momentum equations,

$$
\underline{u}^{n+1}=-\delta \underline{p p}^{n+1}+\underline{u},
$$

gives

$$
\delta t_{V O L} j k \underline{\nabla} \cdot \underline{\nabla} P n+1=V O L_{i} j k \underline{\nabla} \cdot \underline{u} \text {. }
$$

where Eq. (11) ras been multiplied by the cell volume VOLijk to make it conservative and therefore symmetric.

Reference 6 describes procedures for solving equations such as Eq. (11), which may be written in matrix form as

$$
A \underline{s}=\underline{h},
$$

where the vector $\underline{s}$ represents the unknown pressures $P^{n+1}$ and the matrix $A$ is symmetric. These Conjugate Gradient Methods are a family of iteration procedures that attempt to minimize error functionals of the form

$$
E_{\gamma}(\underline{\tilde{s}})=(\underline{s}-\underline{\tilde{s}})^{\top} A^{\gamma}(\underline{s}-\underline{\tilde{s}}) \text {. }
$$

where $\underline{\tilde{s}}$ is an approximate solution obtained by iteration and $\underline{s}$ is the actual solution of Eq. (12). Each integer value of $\gamma$ corresponds to a different member of the family of solution procedures. It can be shown that these Conjugate Gradient schemes are the optimum iteration procedures for whichever norm [Eq. (13)] chosen to test convergence. We choose to use the Conjugate Residual Method, which corresponds to $\gamma=2$ : 
$E_{2}(\underline{\tilde{s}})=(\underline{s}-\underline{\tilde{s}})^{\top} A^{2}(\underline{s}-\underline{\tilde{s}})=r^{\top} r$.

where $r$ is the residual error,

$$
\underline{r}=\underline{h}-\hat{A} \underline{\tilde{s}} .
$$

This method was chosen because $I$ can be related to the velocity divergence field, which is what we wish to minimize most efficiently.

These iteration procedures can be made even more efficient if one preconditions the system of Eqs. (12). He multiply this equation by $0^{-1}$ :

$$
Q^{-1} A Q^{-} Q^{\top} \underline{\underline{S}}=Q^{-1} \underline{h},
$$

where 0 is an unspecified nonsingular matrix that makes the equation easier to solve. Then Eq. (12) becomes

$$
A^{\prime} \underline{s}^{\prime}=\underline{h}^{\prime},
$$

where

$$
\begin{aligned}
& A^{\prime}=Q^{-1} A Q^{-T}, \\
& \underline{S}^{\prime}=Q^{\top} \underline{s}, \text { and } \\
& \underline{h}^{\prime}=Q^{-1} \underline{h}
\end{aligned}
$$

and Eq. (17) is solved by the Conjugate Residual Method as before. We choose $Q$ so that $A$ is well approximated by $M=Q Q^{\top}$; therefore,

$$
A^{\prime} S^{\prime} \approx Q^{-1} Q Q^{\top} Q^{-\top} \underline{s}^{\prime}=\underline{s}^{\prime}
$$


With this approximation, the eigenvalues of $A^{\prime}$ are all close to unity, so the iteration proceeds very rapidly. The matrix $Q$ is obtained by an incomplete Cholesky decomposition.

The convergence of the pressure iteration is tested by comparing the maximum absolute value of the divergence of the velocity field with a small number, $\varepsilon$. Typically, $\varepsilon$ is of the order of $10^{-4}$, but it can vary with the specific problem being solved. When the iterations have converged, the velocity components are corrected with the difference between the $n+1$ and $n$ time-level pressure gradients.

$$
\begin{aligned}
& u_{i, j, k}^{n+1}=u_{i, j, k}^{n}+\delta t\left(P_{i, j, k}^{n+1}-P_{i+1, j, k}^{n+1}-P_{i, j, k}^{n}+P_{i+1, j, k}^{n}\right) / \delta x c_{i} \\
& v_{i, j, k}^{n+1}=v_{i, j, k}^{n}+\delta t\left(P_{i, j, k}^{n+1}-P_{i, j+1, k}^{n+1}-P_{i, j, k}^{n}+P_{i, j+1, k}^{n}\right) / \delta y c, \text { and } \\
& w_{i, j, k}^{n+1}=w_{i, j, k}^{n}+\delta t\left(P_{i, j, k}^{n+1}-P_{i, j, k+1}^{n+1}-P_{i, j, k}^{n}+P_{i, j, k+1}^{n}\right) / \delta z c_{k} .
\end{aligned}
$$

\section{THE PARTICLE DYNAMICS SOLUTION ALGORITHM}

The motion of discrete (solid) particles is computed by SOLA-DM whenever the input variable SOLATYPE is 1.0 or 2.0 (Sec. V.A.5). The particle motion feature may be used to model the movement of solids (for example, dust particles or rock chips) in the fluid.

Sometimes it is desirable to mark specific regions of fluid so that the evolution (that is, location and shape) of these regions can be followed. By setting the fluid dynamic drag coefficient, $c_{d}$, to a very large value (for example, 106), the particles behave as marker particles and track the motion of the fluid. Marker particles also can be useful for computer graphic displays. By adding a capability for particles to diffuse relative to the fluid, they also may be used to model pollutant dispersal or other types of transport in turbulent fluids.

In each of the above cases, the particles do not affect the fluid dynamics. They are passive quantities that move with the mean flow and, if the option is selected, diffuse with the constant diffusion coefficient NUP. 


\section{A. Equations of Motion}

Solid paiticles are modeled in SOLA-DM as individual equivalent spheres of radius $R$. The particle/fluid volume ratio is assumed to be small enough that particle effects on fluid velocities can be neglected. A location $\left(x_{p}, y_{p}, z_{p}\right)$ and velocity $\left(u_{p}, v_{p}, w_{p}\right)$ are stored for each particle. The fluid velocity components $\left(u ;, v_{f}, w_{f}\right)$ at the particle location are computed from the fluid velocity components on the six faces of the cell that contains the particle using a linear interpolation in each direction (that is, tri-linear interpolation). The equations of motion for a particle are

$$
\begin{aligned}
& \frac{D x_{p}}{D t}=u_{p} \quad, \\
& \frac{D y_{p}}{D t}=v_{p} \quad \text {, and } \\
& \frac{D z_{p}}{D t}=v_{p} \quad ; \quad \text { and }
\end{aligned}
$$

$$
\begin{aligned}
& \frac{D u_{p}}{D t}=-\frac{\rho_{f}}{\rho_{p}} \frac{\partial P}{\partial x}+k\left(u_{f}-u_{1}\right)+g_{x}+\frac{\check{\xi}}{x}\left(v_{p}+\Omega x\right)^{2}, \\
& \frac{D v_{p}}{D t}=-\frac{\rho_{f}}{\rho_{p}} \frac{\partial P}{\partial y}+k\left(v_{f}-v_{p}\right)+g_{y}-\frac{\xi u_{p}}{x}\left(v_{p}+2 \Omega x\right), \text { and } \\
& \frac{D w_{p}}{D t}=-\frac{\rho_{f}}{\rho_{p}} \frac{\partial P}{\partial z}+k\left(w_{f}-w_{p}\right)+g_{z},
\end{aligned}
$$

where $\frac{D}{D t}$ (the Lagrangian time derivative) indicates the time rate of change following an individual particle and $P$ is the fluid pressure divided by the fluid density $\left(p / p_{f}\right)$.

The first term on the right side of each equation contains the ratio of fluid density $\left(\rho_{f}\right)$ to particle density $\left(\rho_{p}\right)$ and accounts for accelerations resulting from pressure gradients (buoyancy). The second term in each equation is 
the acceleration resulting from fluid drag forces, and the third term is the acceleration caused by body forces. The fourth terms in the first two equations account for inertial accelerations in a cylindrical coordinate system.

If the density of the particles is equal to the fluid density, the particles should behave almost like fluid parcels, except that they are nondeformable. Thus, in this limit, we should find that the particle equations of motion [Eq. (22)] reduce to the fluid dynamic equations. This limit provides a consistency check on the particle equations. The equations in Eq. (22) are consistent because, if $\rho_{p}$ is set equal to $\rho f$ and the drag term is neglected, the particle equations are identical to the fluid equations. In making this comparison, we must remember that the left sides of Eq. (22) are convective derivatives; for example,

$$
\frac{D u}{D t}=\frac{\partial u}{\partial t}+u \frac{\partial u}{\partial x}+v \frac{\partial u}{\partial y}+w \frac{\partial u}{\partial z}
$$

\section{B. Fluid-Dynamic Drag}

Fluid drag is expressed as a coefficient $k$ times the relative velocity between the fluid $\left(\vec{u}_{f}\right)$ and the particle $\left(\vec{u}_{p}\right)$ :

$$
\vec{D}=k\left(\vec{u}_{f}-\vec{u}_{p}\right)
$$

where $\vec{D}$ is the drag force per unit mass. Except for very small particles (for which a Stokes drag estimate is permissible), k will be a function of the relative velocity magnitude. Unfortunately, an exact expression for the drag coefficient $C_{d}$ that applies to irregularly shaped particles is not available. We assume a combination of Stokes drag and form drag.

1. Stokes Drag. For a Reynolds number $(R e)<10$, the fluid drag force $\left(\dot{F}_{d}\right)$ on spherical (and approximately spherical) particles is given by Stokes' law:

$$
\vec{F}_{d}=6 \pi \mu R\left(\vec{u}_{f}-\vec{u}_{p}\right)
$$


where $R$ is the nominal radius of the particle and $\mu$ is the laminar dynamic coefficient of viscosity. Then, the fluid drag force per unit mass (acceleration) is found by dividing Eq. (25) by the mass of the sphere:

$$
\vec{D}=\frac{6 \pi \mu R\left(\vec{u}_{f}-\vec{u}_{p}\right)}{\rho_{p} \frac{4}{3}^{\pi R^{3}}}=\frac{9_{\mu}\left(\vec{u}_{f}-\vec{u}_{p}\right)}{2 \rho_{p} R^{2}} .
$$

Equating Eqs. (24) and (26) yields an expression for $k$ for small Re:

$$
k=\frac{9 p_{f} \mu}{2 p_{p} \rho_{f} R^{2}}=\frac{9 p_{f} \nu_{f}}{2 p_{p} R^{2}} .
$$

where $v_{f}$ is kinematic coefficient of viscosity.

2. Form Drag. For higher $R \Xi$, an appropriate expression for $D$ is

$$
\vec{D}=\frac{\frac{1}{2} \rho_{f}\left(\vec{u}_{f}-\vec{u}_{p}\right)\left|\vec{u}_{f}-\vec{u}_{p}\right| A_{\rho} C_{d}}{\rho_{p} \frac{4}{3} \pi R^{3}}
$$

where $A_{p}$ is the projected area of the particle in the direction of $\left(\vec{u}_{f}-\vec{u}_{p}\right)$. Equating Eqs. (24) and (28) yields an expression for $k$ for $10^{3}<\operatorname{Re}<10^{5}$ :

$$
k=\frac{3}{8} \frac{\rho_{f}}{\rho_{p}} \frac{C_{d}}{R}\left|\vec{u}_{f}-\vec{u}_{p}\right| .
$$

An effective drag function that includes Stokes drag and form drag is obtained by combining Eqs. (27) and (29): 


$$
k=\frac{\rho_{f}}{\rho_{p}} \frac{9 v_{f}}{2 R^{2}}+\frac{3 C_{d}}{8 R}\left|\vec{u}_{f}-\vec{u}_{p}\right|
$$

For spheres, $C_{d} \sim 0.47$ for $10^{3}<\operatorname{Re}<2 \times 10^{5}$; however, choosing a s'ditable $C_{d}$ for irregular particles is more difficult.

Particles that cross continuative or constant pressure boundaries are deleted, but particles crossing a periodic boundary are reinserted at the opposite side of the mesh. At rigid walls (including internal obstacles), particles are reflected specularly.

\section{Particle Diffusion}

When the particle diffusion coefficient, NUP, is not zero, each particle is given an additional increment in position, which is added to the mean flow increment before the new coordinate calculation and boundary condition tests are performed. The idea behind this diffusion increment is to first consider the particle as a point source. After a time interval, $\delta t$, this point should diffuse in all directions, forming a small cloud with a Gaussian mass distribution in each direction (a "Gaussian puff"). However, the particle is to remain a pcint. Therefore, rather than replacing it with a cloud, the Gaussian cloud is interpreted as a probability distribution for moving the particle; that is, using a random number generator, a new location for the particle is selected with a probability corresponding to the mass distribution within the cloud. In this way, a Monte Carlo approximation is generated for the local diffusion of each particle. (By definition, many repetitions of the random shift routine eventually would produce the Gaussian cloud distribution.) In the code, three random numbers are selected for each particle to compute shifts in each of the three coordinate directions. Repeating this process over many time steps, together with the mean flow advection, will produce a solution for the transport equation,

$$
\frac{\partial \rho}{\partial t}+u \frac{\partial \rho}{\partial x}+v \frac{\partial \rho}{\partial y}+w \frac{\partial \rho}{\partial z}=\operatorname{NUP}\left(\frac{\partial^{2} \rho}{\partial x^{2}}+\frac{\partial^{2} \rho}{\partial y^{2}}+\frac{\partial^{2} \rho}{\partial z^{2}}\right)
$$

in which $\rho$ is the concentration of marker particles. The accuracy of the solution will improve as the number of particles per unit volume is increased. 
The user easily can extend this simple transport model to allow NUP to assume space- and time-dependent values and to even allow NUP to assume different values in each coordinate direction. These changes are made by using the appropriate values for NUP in the subrout ines PARMOV and PARTDIFF.

Two additional points regarding the Monte Carlo diffusion approximation should be noted. First, the random number generator used in the code is based on a uniform distribution in the interval $(0,1)$. This number, $x$, is transformed to a uniform distribution in $(-1,1)$ by the arithmetic operation $2 x-1$; an inverse error function then must be computed to get the desired Gaussian distribution. Unfortunately, system routines for inverse error functions are time consuming and, when used three times for every particle in every time step, can increase computational costs significantly. To minimize this problem, SOLA-DM creates a table of inverse error functions with 101 entries covering the error function argument from 0 to WMAX. The default value for WMAX is 2.0, and experience has showi this to be sufficiently accurate for most applications. However, the user may input a different value of WMAX in NAMELIST/PARTS/. In the particle diffusion routine, PARTDIFF, this table is interpolated for the inverse error function for each random number, which requires much less time than the more accurate system routine.

A second point to note is that a call is made to a system clock at the be-ginning of routine RPARTS, which initializes the random number generator. This ensures a new random number sequence with each new calculation.

For some applications, the user may wish to run particle transport calculations in a steady flow, for example, to investigate pollutant dispersal sensitivity to different source locations or different diffusion rates. In such cases, it makes no sense to continue the fluid dynamic solution portion of the code. By setting the parameter SOLATYPE $=2.0$ in NAMELIST/XPUT/, the code will continue to update time and cycle counters but will be cycling only through its particle moving sections. Thus, the flow field will be frozen, and a considerable savings in computer time will be realized.

\section{Outline of Numerical Solution Method}

The basic procedure for advancing the particle transport solution through one increment in time, $\delta t$, consists of four steps for each particle.

(1) Compute the fluid velocity at the particle location. This local fluid velocity, which is used to compute fluid drag force, has two 
parts: the velocity interpolated from the complaced velocity field (Sec. $11, D)$ and a diffusion velocity computed by a Monte-Carlo method (Sec. $111, \mathrm{C})$.

(2) Explicit approximations of the momentum equations [Eqs. (22)] without fluid drag forces are used to compute intermediate particle velocities. Fluid pressure and rotational accelerations are taken from previous time-level values.

(3) Include the effects of fluid drag and compute a new time-level particle velocity. Because the drag function [k in Eqs. (22)] is a function of the relative velocity between the fluid and the particle, an iterative procedure is used to approximate particle velocity.

(4) Move the particle to the new time-level spatial location [Eqs. (21)] using the average particle velocity for time increment $\delta t$.

\section{E. Approximate Particle Motion Equations}

For each particle, the finite-difference approximation to Eqs. (22) without fluid drag terms is

$$
\begin{aligned}
& \tilde{u}_{p}=u_{p}^{n}+\delta t\left[-\frac{\rho_{f}}{\rho_{p}}\left(\frac{\partial p}{\partial x}\right)^{n+1}+g_{x}+\frac{\left.\xi\left(v_{p}^{n}+\Omega x_{p}^{n}\right)^{2}\right]}{x_{p}^{n}}\right], \\
& \tilde{v}_{p}=v_{p}^{n}+\delta t\left[-\frac{\rho_{f}}{\rho_{p}}\left(\frac{\partial p}{\partial y}\right)^{n+1}+g_{y}-\frac{\xi u_{p}}{x_{p}^{n}}\left(v_{p}^{n}+2 \Omega x_{p}^{n}\right)\right], \text { and } \\
& \tilde{w}_{p}=w_{p}^{n}+\delta t\left[-\frac{\rho_{f}}{\rho_{p}}\left(\frac{\partial p}{\partial z}\right)^{n+1}+g_{z}\right],
\end{aligned}
$$

where the tilde indicates intermediate values for the components of the particle velocity.

The fluid pressure gradient components are computed from the pressures in cells near the particle; for example, if $X C_{i} \geq x_{p}>X C_{i+1}$, then $\partial P / \partial x$ at time level $n+1$ is approximated as 
$\left(P_{i+1, j, k}^{n+1}-P_{i, j, k}^{n+1}\right) / \delta X C_{i}$

Next, the effects of fluid drag are included in computing the time-level $n+1$ values for $u_{p}, v_{p}$, and $w_{p}$. The intermediate paricle velocity components calculated in Eqs. (31) are used as initial guesses in a Newton-Raphson iteration procedure. In Eqs. (32) and (33), the subscripts $m$ and $m+1$ indicate successive iterates for the $n+1$ time-level values. The subscript $p$ has been dropped for clarity.

$$
\begin{aligned}
& k=\frac{\rho}{\rho_{p}}\left\{\frac{9 v_{f}}{2 R^{2}}+F_{d} \sqrt{\left(u_{f}-u_{m}^{n+1}\right)^{2}+\left(v_{f}-v_{m}^{n+1}\right)^{2}+\left(w_{f}-w_{m}^{n+1}\right)^{2}}\right\}, \\
& u_{m+1}^{n+1}=u_{m}^{n+1}-\left[\frac{u_{m}^{n+1}-\tilde{u}-(\delta t)(k)\left(u_{f}-u_{m}^{n+1}\right)}{1+\delta t\left(k+F_{d}\left|u_{f}-u_{m}^{n+1}\right|\right)}\right], \\
& v_{m+1}^{n+1}=v_{m}^{n+1}-\left[\frac{v_{m}^{n+1}-\tilde{v}-(\delta t)(k)\left(v_{f}-v_{m}^{n+1}\right)}{1+\delta t\left(k+F_{d}\left|v_{f}-v_{m}^{n+1}\right|\right)}\right], \text { and } \\
& w_{m+1}^{n+1}=w_{m}^{n+1}-\left[\frac{w_{m}^{n+1}-\tilde{w}-(\delta t)(k)\left(w_{f}-w_{m}^{n+1}\right)}{\left.1+\delta t\left(k+F_{d} \mid w_{f}-w_{m}^{n+1}\right]\right)}\right],
\end{aligned}
$$

where $F_{d}=\frac{3}{8} \frac{C_{d}}{R}$.

Finally, the discrete form of Eqs. (21) is computed to find the new timelevel particle locations. An average particle velocity is used:

$$
\begin{aligned}
& x_{p}^{n+1}=x_{p}^{n}+\delta t\left(u_{p}^{n}+u_{p}^{n+1}\right) / 2, \\
& y_{p}^{n+1}=y_{p}^{n}+\delta t\left(v_{p}^{n}+v_{p}^{n+1}\right) / 2, \text { and }
\end{aligned}
$$




$$
z_{p}^{n+1}=z_{p}^{n}+\delta t\left(w_{p}^{n}+w_{p}^{n+1}\right) / 2 .
$$

IV. THE SOLA-DM FORTRAN PROGRAM

\section{A. Code Structure (Subroutine List)}

SOLA-DM is written in subroutine form such that each subroutine performs a separate logical task. Subroutine names have been selected to indicate the function that each performs, and these are arranged alphabetically. The following list gives a brief description of the tasks performed by each subroutine.

\section{ALFOMEGA (Beginning and Ending)}

(1) Computes beginning and ending mesh indices for $D 0$ loops.

\section{BC (Boundary Conditions)}

(1) Driver for setting boundary conditions on the six bounding surfaces of the computational mesh.

\section{BCFLUX (Boundary Flux)}

(1) Computes the net volume flux into the computational domain.

(2) Applies a correction to all continuative and specified pressure boundaries to make the net volume flux equal to zero.

\section{BCSET (Set Boundary Conditions)}

(1) Problem-dependent boundary conditions are inserted here.

\section{BCX (Boundary Conditions in $\times$ Direction)}

(1) Sets boundary conditions at the two " $y-z$ " boundary surfaces.

BCY (Boundary Conditions in y Direction)

(1) Sets boundary conditions at the two " $x-z$ " boundary surfaces.

BCZ (Boundary Conditions in z Direction)

(1) Sets boundary conditions at the two " $x-y$ " boundary surfaces.

\section{BETACAL (BETA Calculation)}

(1) Calculates a flag (IBETA) for each cell to identify obstacles, walls, or mesh boundaries.

(2) Computes the area open to flow at all continuative and specified pressure boundaries. 


\section{CELLOIV (Divergence Operator)}

(1) Computes the divergence of a vector field.

\section{CNTR (Contour Plot)}

(1) Plots the pressure (or other) contours on film.

\section{DELTADJ (Time-step Control)}

(1) Computes the maximum allowable time step, DELT, for numerical stability.

(2) Adjusts the time step according to the number of pressure iterations. DGAP (Define Graph Area for Perspective Plots)

(1) Defines the plot boundaries for perspective view plots.

\section{DRAW (Control for Graphics Output)}

(1) Calls VeLV to draw velocity vector plots.

(2) Calls PARPLT to draw marker particle plots.

(3) Calls CNTR to draw contour plots.

\section{DRAWLINE (Draw a Line)}

(1) Draws a line from point "A" to point "B."

\section{DRAWLV (Draw Lines or Arrows)}

(1) Calls DRVG to draw I ine segments or GVECT to draw vectors. DRAMMESH (Draw the Computational Mesh)

(1) Draws the mesh for any surface in the computational domain. DRAWPOLY (Draw a Polygon)

(1) Draws an arbitrary-sided polygon.

\section{DRAWNECT (Draw a Vector)}

(1) Draws a vector from point "A" to point "B." DRFP (Draw a Frame around Perspective Plots)

(1) Draws a frame around perspective view plots.

\section{DRFPDIV (Driver for Perspective Plots)}

(1) Driver for drawing mesh and obstacle outlines in perspective views. 
EXTRACT (Extract Computed Data)

(1) Extracts vertex-centered values on specified logical surfaces.

\section{FLUXFACE (Flux Through Cell Faces)}

(1) Computes a "flow" or "nomflow" condition for each cell face from the IBETA flag.

\section{FSCALE (Scalo a Variable)}

(1) A variable $v$ in the range $v_{1} \leq v \leq v_{2}$ is mapped to $f$, where $f_{1} \leq f \leq f_{2}$.

\section{GETPLANE (Get Plane Type)}

(1) Determines whether a given plane is normal to the $x, y$, or $z$ direction.

\section{GLOSSARY (Glossary of Code Variables)}

(1) Lists most of the variables used in the code.

(2) Describes the indexing convention used for cells, vertices, and faces.

\section{GRADPP (Pressure Gradient for Particles)}

(1) Computes the fluid pressure gradient components acting on each particle.

\section{GRIDBOX (Draw an $x-y$ Grid BOx)}

(1) Draws an $x$-y grid box, labels the coordinate axes, and puts grid tick marks on all four sides of the box.

INDEX (Logical Indices)

(1) Computes mesh indices and strides for out lining mesh surface.

INDEXD (Indices on Mesh Diagonals)

(1) Computes indices in $x-y$ planes used for diagonal sweeps in subroutine PRESSURE.

\section{LOADINT (Load an Integer Vector)}

(1) Loads the same integer value into all elements of a vector.

\section{LOADREAL (Load a Real Vector)}

(1) Loads the same real value into all elements of a vector.

\section{LPRT (Long Print)}

(1) Prints dependent variable values for all cells on film or paper. 
MESHMAP (Map of Computational Mesh)

(1) Prints out a table listing important information about each cell. MESHINDX (Mesh Indices)

(1) Calculates the absolute mesh index $M$ from the logical indices 1, J, and $\mathrm{K}$.

(2) Calculates the logical indices $1, J$, and $K$ from the absolute mesh index $M$.

\section{MESHSET (Mesh Setup)}

(1) Sets up computer memory storage requirements for the mesh.

(2) Generates the computing mesh from input data by calling MESHX. MESHY, and MESHZ.

(3) Computes some geometric quantities used in the code.

\section{MESHSIZE (Mesh Physical Size)}

(1) Computes the physical dimensions of any surface or logically rectangular subsurface in the computational domain.

\section{MESHX (X Direction Mesh Setup)}

(1) Computes the $x$ coordinates for mesh 1 ines and cell centers.

(2) Computes cell sizes and reciprocals of all quantities needed in the code.

\section{MESHY (Y Direction Mesh Setup)}

(1) Similar to MESHX but for the y direction.

\section{MESHZ ( $Z$ Direction Mesh Setup)}

(1) Similar to MESHX but for the z direction.

\section{NABORS (CeII Neighbors)}

(1) Computes the of fsets needed to reference the 26 cells adjoining mesh cell $\mathrm{M}$.

\section{NEWPROB (New Problem)}

(1) Driver for setting up a new problem.

\section{OUTPUTS (Problem Outputs)}

(1) Controls the printed and graphics output.

(2) Controls the tape dump output. 
PARMOV (Particle Movement)

(1) Computes the movement of solid particles in the fluid and allows for particle diffusion.

(2) Creates or destroys particles flowing into or out of the mesh.

PARTDIFF (Particle Diffusion)

(1) Computes particle diffusion velocities.

\section{PARTPLT (Particle Plot)}

(1) Plots particles in perspective.

\section{PARTCELL (Particle Cell Location)}

(1) Computes the absolute mesh cell location of particles.

\section{PARTSET (Particle Setup)}

(1) Sets up a parallelepiped volume of particles in problem units using input data.

(2) Computes initial velocity for each particle.

PCNV (Perspective Coordinate Transformation)

(1) Converts a three-dimensional point to perspective (image plane) coordinates.

\section{PLABEL (Plot Labels)}

(1) Writes labels on all two-dimensiorial plane and perspective plots.

\section{PLOTSIZE (Plotting Window)}

(1) Computes the horizontal and vertical bounds for two-dimensional plots.

PRESSIT (SOR Pressure Iteration--Obsolete Subroutine)

(1) Iteratively adjusts pressures and velocities to satisfy incompressibility condition.

(2) Computes free surface cell pressures.

\section{PRESSURE (Conjugate Gradient Pressuro)}

(1) Iteratively adjusts pressures and velocities using the Preconditioned Conjugate Gradient Method.

\section{RDTAPE (Read Tape)}

(1) Reads restart file RSSOLA, which must be written as file TAPE8 by routine WRTAPE. 


\section{RECIP (Reciprocal)}

(1) Computes the reciprocal of each element of a vector.

\section{RESTART (Restart a Problem)}

(1) Driver for restarting a calculation.

\section{RESTORE (Restore Graphics)}

(1) Restores CGS and CGSHIGH graphics environment to default values.

\section{RGRAFIC (Read Graphics Input Data)}

(1) Reads input data for graphics displays from NAMELIST/GRAFIC/.

\section{RINPUT (Read Input Data)}

(1) Sets default values for problem input data.

(2) Reads problem input data from NAMELIST/XPUT/.

\section{ROUND (Round a Number)}

(1) Rounds or truncates each element of an array to a specified number of significant digits.

\section{RPARTS (Read Particle Setup Data)}

(1) Reads input data for marker particle setup from NAMELIST/PARTS/. Input is in problem units.

(2) Initializes random number generator used for particle diffusion.

(3) Loads inverse error function table for particle diffusion.

\section{SECORD (Second-Order Approximation Option)}

(1) Shifts velocity arrays as needed to exercise second-order accurate option.

\section{SETGNDX (Set Grid Indices)}

(1) Check the mesh index bounds (input for graphics) for consistency.

\section{SETUP (Set Initial Conditions)}
(1) Sets constant parameters used in code.
(2) Computes viscous diffusion time step.
(3) Sets initial velocities into velocity arrays.
(4) Calls VOLUME to compute cell volumes. 
(5) Calls BETACAL to set IBETA flag array.

(6) Calls BC and DELTADJ to initialize boundary conditions and time step.

(7) Calls DRAWMESH to plot a two-dimensional slice of the mesh in each of the three coordinate directions.

\section{SOLA (Basic Solution Algorithm)}

(1) Advances time and cycle number.

(2) Calls BC to set boundary conditions.

(3) Calls TILDE to explicitly advance velocities to time level $n+1$.

(4) Calls PRESSURE to iteratively adjust pressures and velocities to satisfy the incompressibility condition.

(5) Calls UPDATE to set advanced time variables into previous time arrays in preparation for next cycle.

(6) Calls DELTADJ to reset the time step.

(7) Calls PARMOV to move particles.

(8) Calls OUTPUTS for paper, film, and tape dump outputs.

(9) Tests for end of problem.

\section{SOLADM (Main Program)}

(1) Calls START to initialize memory and graphics libraries.

(2) Calls RINPUT to read in basic problem data.

(3) Calis NEWPROB or RESTART to set up a new problem or to restart a previous calculation.

(4) Calls the basic solution algorithm SOLA.

(5) Calls WRAPUP to end the calculation.

\section{START (Initialize Memory Constants)}

(1) Sets some constants

(2) Sets film package, which is specific to each computer installation.

\section{TILDE (Advance Velocities in Time)}

(1) Computes explicit velocity advancement using time $n$ values for all contributions to momentum equations. 


\section{TIMING (CPU Timing Information)}

(1) Writes out the CPU seconds and computing speed (in megaflops) for the major subroutines. (This is done at the end of the calculation.)

\section{UPDATE (Update Arrays)}

(1) Set the advance time values for velocity components and surface height into the time-in arrays.

\section{VELV (Velocity Vector Plot)}

(1) Draws velocity vectors in plane or perspective views.

(2) Writes maximum velocity values on the plot.

\section{VERTEX (Compute Vertex-Centered Values)}

(1) Computes the vertex-centered value of any variable based on a volumeweighted average of the surrounding eight cell-centered values.

\section{VMINMAX (Minimum and Maximum Values)}

(1) Finds the minimum and maximum values of an array

(2) Finds the minimum and maximum absolute values of an array.

\section{VOLUME (Cell Volumes)}

(1) Computes the volume of all mesh cells.

\section{WRAPUP (Conclude the Calculation)}

(1) Performs housekeeping at the end of a calculation.

\section{WRTAPE (Write Tape File)}

(1) Write restart dump file TAPE8 at selected times.

\section{XYCOORD ( $x$ and $y$ Coordinates)}

(1) Computes $x$ and $y$ cartesian coordinates corresponding to r, rc, and $s$ curvilinear coordinates.

\section{B. Single Indexing Scheme}

For computational purposes, we represent the location of each cell by a single index $M$ that takes on integer values from 1 (corresponding to $1=1$, $J=1$, and $K=1$ ) to MSIZE (corresponding to $I=I M A X, J=J M A X$, and $K=K M A X$ ). 
The general relationship between the absolute mesh index $M$ and the logical coordinate indices $\mathrm{I}, \mathrm{J}$, and $\mathrm{K}$ is

$$
M=1+(J-1) * I \operatorname{MAX}+(K-1) * I \operatorname{MAX} * J \operatorname{MAX} .
$$

Geometrically, this formula says that

(1) a unit shift in I causes a \pm 1 change in $M$,

(2) a unit shift in $J$ causes a \pm IMAX change in $M$ (one row of cells), and

(3) a unit shift in $K$ causes a \pm IMAX * JMAX change in $M$ (one layer of cells).

Section II.B describes the labeling convention whereby the velocity components on the three cell faces with positive outward normals have the same indices as the cell. It then follows that the vertex common to all three positive faces is identified with cell $M$ as shown in Fig. 5. The other seven vertices are identified with neighbor cells. When referencing any of the 26 nearest neighbors that share a common face, edge, or vertex with $M$, we use a shorthand

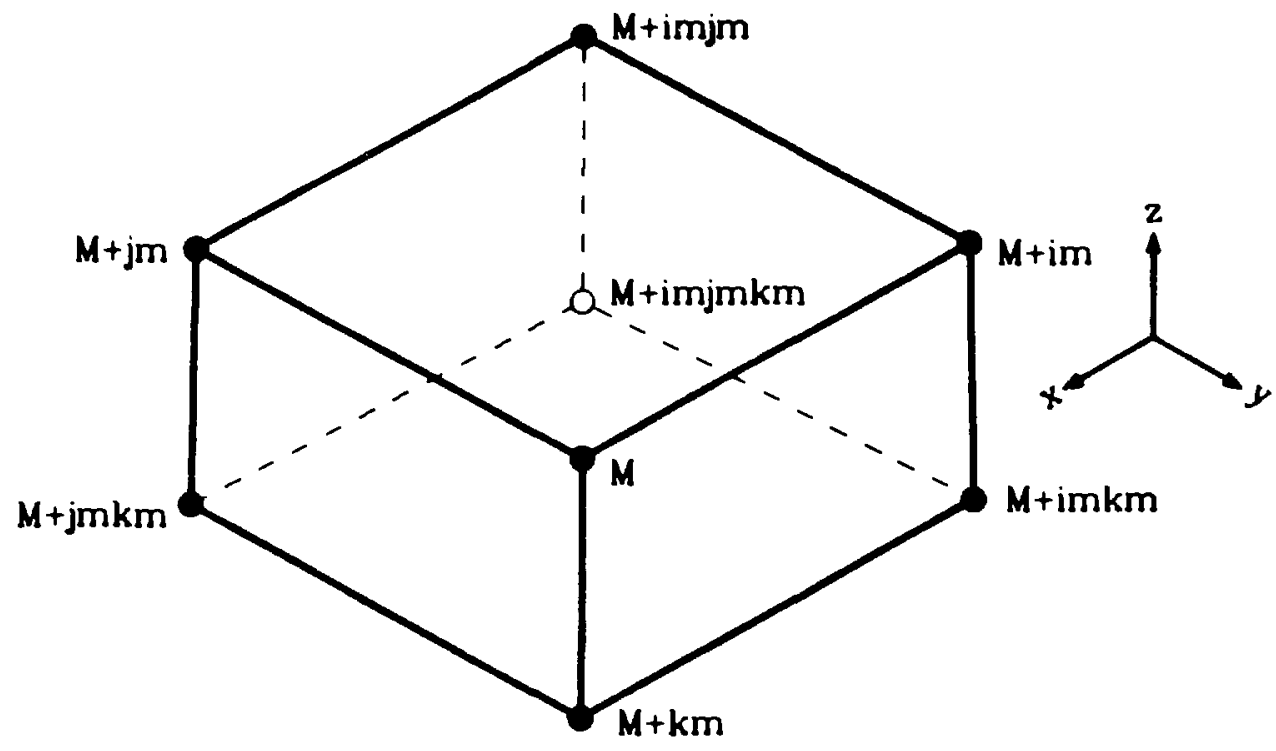

Fig. 5 .

Single indexing convention. An arbitrary mesh cell $M$ is identified with the vertex common to the three faces with positive outward normals. Offsets from $M$ are used to locate other cells that have a vertex in common with cell $M$. 
notation where "p" stands for " + " and " $m$ " stands for "-". For example, we write $M$ in place of $(I, J, K)$. Similarly, more complex quantities like $M+$ imkp and $M+$ jpkm are written in place of $(1-1, J, K+1)$ and $(I, J+1, K-1)$, respectively. offsets are computed during problem setup in subrout ine NABORS.

\section{Automat ic Mesh Generator}

SOLA-DM uses a mesh composed of rectangular cells with variable sizes. Although the coordinates of each mesh line could be read in for every new problem, this is highly inconvenient in most cases. To circumvent this, the code contains an automatic mesh generator that is general enough to satisfy almost all requirements. The basic idea is to build a mesh by stacking together a series of submeshes in each coordinate direction. For example, consider the $x$ direction. The $x$ dimension of the problem to be solved is subdivided into a set of NKX intervals. The kth interval extends from its left end, $X L(k)$, to the left end of the next interval, XL $(k+1)$. Within each interval there is a location, $X C(k)$, where the mesh cells will be smallest ("XC" for convergence point). The number of cells between $X L(k)$ and $X C(k+1)$ is specified as $N X L(k)$, and the number from $X C(k)$ to $X L(k+1)$ is specified as $N X L(k)$. The minimum cell size, which is located at $X C(k)$, is specified as $\operatorname{DXMN}(k)$.

Using the above information, the mesh generator expands cell sizes from a value of DXMN $(k)$ at $X C(k)$ in a quadratic manner such that the required number of cells will lie on each side of XC(k) and fill the subinterval. If DXMN(K) is larger than the cell size corresponding to uniform zoning, then uniform zoning is produced by the generator.

The number of cells defined on either side of $x C(k)$ can be any value, including zero. The latter choice is often useful when the minimum cell size is wanted at the beginning or end of a subinterval.

Any number of submeshes may be stacked together provided sufficient storage has been allowed for the necessary input data arrays. Thus, it is possible to generate complicated meshes with locally fine resolution around any number of points. Furthermore, there should be no unexpected numerical stability problems because minimum cell sizes are specified as part of the input data.

In summary, the input parameters for mesh subdivision (k) in the $x$ direction are as follows.

NKX specifies the number of subdivisions in the $x$ direction.

$X L(k)$ sets the location of the left boundary of subdivision $(k)$. 
$X C(k)$ sets the "convergence point" about which the cell spacing is the minimum value in subdivision ( $k$ ).

NXL(k) specifies the number of cells between locations $X L(k)$ and $X C(k)$ in subdivision ( $k$ ).

$\operatorname{NXR}(k)$ specifies the number of cells between locations XC(k) and XL(k+1) in subdivision ( $k$ ).

DXMN(k) specifies the minimum space increment in the $x$ direction in subdivision $(k)$.

An analogous treatment is used in the $y$ and $z$ directions except for notation changes.

$x$ direction: $\quad \operatorname{NKX}, X L(k), X C(k), N X L(k), N X R(k), \operatorname{OXMN}(k)$

y direction: NKY, YL(k), YC(k), NYL(k), NYR(k), OYMN(k)

$z$ direction: NKZ, ZL(k), ZC(k), NZL (k), NZR(k), DZMN(k)

When periodic conditions are specified (for example, in the $x$ direction), there must be two cells reserved at the right end of the mesh for setting periodic boundary conditions. It also is necessary that these cells have the same sizes as the first two interior cells on the left side of the mesh to insure periodicity. The mesh generator does this automatically, so the user only needs to input the dimensions of the periodic interval he wishes to use. However, in setting storage requirements, it must be remembered that periodic meshes need three rather than two additional cells for boundary conditions. Likewise, for periodic conditions in the y direction, there will be two extra cells at the maximum $j$-index end of the mesh; for the z-direction periodicity, there will be two extra cells at the maximum $k$-index end. In the case of cylindrical coordinates $(r, \theta$, and $z)$, the values of $Y L(k)$ and $Y C(k)$ are in degrees. A complete list of mesh line coordinates, cell sizes, and other geometric data is output by the code at the beginning of each new calculation.

To illustrate the use of this mesh generator, consider the following simple, one-dimensional examples.

1. A uniform mesh in the $x$ direction extending from $x_{1}$ to $x_{2}$ and containing $N$ cells is defined by 


$$
\begin{aligned}
\operatorname{NKX} & =1, \\
X L(1) & =x_{1}, \quad X L(2)=x_{2}, \\
X C(1) & =x_{1}, \\
N X L(1) & =0, N X R(1)=N, \text { and } \\
\operatorname{DXMN}(1) & =x_{2}-x_{1} \text { (or any large value). }
\end{aligned}
$$

2. A symmetric mesh about $x=0$ extending from -10.0 to +10.0 with cell size 0.1 at $x=0$ and containing 20 cells (average cell size: 1.0 ) would be set up with the input data

$$
\begin{aligned}
\operatorname{NKX} & =1, \\
\operatorname{XL}(1) & =-10.0, \quad X L(2)=+10.0, \\
X C(1) & =0.0, \\
\operatorname{NXL}(1) & =10, \operatorname{NXR}(1)=10, \text { and } \\
\operatorname{DXMN}(1) & =0.1 .
\end{aligned}
$$

The generated mesh in this case would have its smallest cells at $x=0$ and its largest cells at either end. The largest cells have size 1.9. This size always can be computed by remembering that, within any interval $[X L(k), X C(k)]$ or $[X C(k), X L(k+1)]$, the minimum, $\delta X_{\min }$, and maximum, $\delta X_{\max }$, cell sizes are related by

$$
\left(\delta x_{\min }+\delta X_{\max }\right) / 2=\delta X_{A}
$$

where $\delta X_{A}$ is the average cell size in the interval, that is,

$[X C(k)-X L(k)] / N X L(k)$ or $[X L(k+1)-X C(k)] / N X R(k)$.

\section{Boundary Conditions}

A complete fluid dynamics integration cycle requires that we update the velocity and pressure at all mesh boundaries and at the surfaces of all internal obstacles.

1. Mesh Boundaries. At the mesh boundaries, a variety of conditions may be set using the layer of fictitious cells surrounding the mesh. For example, 
consider the boundary separating the $i=1$ and $i=2$ layer of cells. The $i=1$ cells are fictitious in the sense that variable values are set in these cells to satisfy boundary conditions rather than calculated according to the finitedifference expressions used at a!l interior mesh locations. If this boundary is to be rigid wall, the normal velocity there must be zero. The tangential velocity can be set to zero for a no-slip type of wall, or the normal derivative of the tangential velocity can be set to zero, corresponding to a free-slip boundary condition. Thus, for a free-slip, rigid wall, the boundary conditions for all $j, k$ are

$$
\begin{aligned}
& u_{1, j, k}=0.0, \\
& v_{1, j, k}=v_{2, j, k}, \\
& w_{1, j, k}=w_{2, j, k}, \text { and } \\
& P_{1, j, k}=P_{2, j, k}-\left[\left(P_{3, j, k}-P_{2, j, k}\right) / d x_{2}\right] d x c_{1} .
\end{aligned}
$$

Because the velocity values are set in boundary cells, the pressure in boundary cells does not enter into the solution of the momentum equations [Eq. (4)]. However, an accurate value for the pressure in boundary cells is needed to compute the fluid pressure gradient acting on particles located near the boundary. Setting $P_{1, j, k}=P_{2, j, k}$ implies $\partial P / \partial x=0$, which may be seriously in error, especially in rotating flows where $\partial P / \partial x=v 2 / x$ even if $u=w=0$. For this reason, we compute $P_{1, j, k}$ as a linear extrapolation of the two adjacent cell pressures normal to the boundary. For a no-slip wall, the conditions are the same except for $u$ and $w$, which must be replaced by

$$
\begin{aligned}
& v_{1, j, k}=-v_{2}, j, k \text { and } \\
& w_{1, j, k}=-w_{2}, j, k .
\end{aligned}
$$


These conditions are imposed on the velocities obtained from the momentum equations and after each pass through the mesh during the pressure iteration.

For a specified in-or out-flow boundary, the specified values for $u, v$, and $w$ should be set in the $i=1$ layer of cells. This must be done as a code modification because no input data have been included for this purpose. It also should be noted that specified in-or out-flow can be different at each j,k (that is, $y, z$ ) location. This flexibility allows an extremely wide range of possible boundary conditions.

Continuative out-flow boundaries (where fluid is to flow smoothly out of the mesh, causing no upstream effects) are always a problem for incompressible flow calculations. Potentially, any prescription can affect the entire flow field. In SOLA-DM, the continuative boundary conditions used at the $i=1$ boundary are the vanishing of all normal derivatives, which are for all $j, k$ :

$$
\begin{aligned}
& u_{1, j, k}=u_{2, j, k}, \\
& v_{1, j, k}=v_{2, j, k}, \\
& w_{1, j, k}=w_{2, j, k}, \text { and } \\
& P_{1, j, k}=P_{2, j, k}-\left[\left(P_{3, j, k}-P_{2, j, k}\right) / d x_{2}\right] d x_{1} .
\end{aligned}
$$

However, these conditions are imposed only after applying the momentum equations and not after each pass through the pressure iteration. Omitting these conditions during the pressure adjustment provides additional "softness" at the boundary that helps to minimize upstream effects. When using this boundary condition, the user should be careful to insure that it is adequate for his purposes.

For periodic boundary conditions in the $x$ direction, both the $i=1$ and $i=$ IMAX boundaries must be set to reflect the periodicity. This is most easily done when the period length is chosen equal to the distance from the boundary between the $i=1$ and 2 cells to the boundary between the $i=\mid \operatorname{MAX}-2$ and IMAX-1 cells. That is, two layers of cells, $i=I \operatorname{MAX}$ and $i=I \operatorname{MAX}-1$, are reserved for setting periodic boundary conditions at the far side of the mesh. 
When the mesh generator is used (Sec. IV.C), this is taken into account automatically and requires no special consideration on the part of the user. The conditions for periodic flow in the $x$ direction are then, at $i=1$ for all $j, k$,

$$
\begin{aligned}
& u_{1, j, k}=u_{I M 2, j, k}, \\
& v_{1, j, k}=v_{I M 2, j, k}, \text { and } \\
& w_{1, j, k}=w_{I M 2, j, k}
\end{aligned}
$$

and at IM1 or IMAX for all j,k,

$$
\begin{aligned}
& u_{1 M 1, j, k}=u_{2}, j, k, \\
& v_{\mid M 1, j, k}=v_{2}, j, k, \\
& w_{1 M 1, j, k}=w_{2}, j, k, \\
& P_{I M 1, j, k}=P_{2, j, k} \text {, } \\
& v_{I M A X}, j, k=v_{3}, j, k \text {, and } \\
& \text { wIMAX, } j, k=w_{3}, j, k \text {, }
\end{aligned}
$$

where IM1 = IMAX -1 and IM2 = IMAX -2 . In this case, these conditions are imposed on velocities computed from the momentum equations and after each pressure iteration. A constant pressure boundary condition at the $i=1$ boundary is set by keeping the pressure in the $i=2$ layer of cells constant and otherwise treating the boundary as continuative. Boundary conditions similar to these are used at all other mesh boundaries. The appropriate normal and tangential velocities must be used in each case.

For convenience, the SOLA-DM program has been written so that any of the above boundary conditions can be imposed by setting input numbers. For example, for the $i=1$ boundary, the quantity WL is an integer set according to this table. 


$\begin{array}{ll}\text { WLL } & \text { Boundary Condition } \\ 1 & \text { Rigid, free-slip wall } \\ 2 & \text { Rigid, no-slip wall } \\ 3 & \text { Continuative } \\ 4 & \text { Periodic in } x \\ 5 & \text { Specified pressure }\end{array}$

In a similar fashion, the other boundaries are controlled by the input parameters.

\begin{tabular}{|c|c|}
\hline Paramete & Mesh Boundary \\
\hline WL & $i=1$ \\
\hline WR & $i=I$ MAX \\
\hline WF & $j=1$ \\
\hline WBK & $j=J M A X$ \\
\hline WB & $k=1$ \\
\hline WT & $k=K M A X$ \\
\hline
\end{tabular}

As previously noted, specified in- or out-flow boundary conditions must be inserted into the program by the user. These and other special conditions should be added in the location reserved for this purpose in boundary condition subroutine BCSET.

2. Internal Obstacle Boundaries. Obstacles within a mesh are defined by flagging those mesh cells that define the obstacles. In SOLA-DM, the IBETA flag array is used to identify obstacles and walls. The user defines which cells are obstacles and which surfaces are walls, and the code automatically assigns the values of IBETA to model this input. The user can modify the IBETA array in the space provided in subroutine BETACAL. For each cell, the three positive cell faces are open or closed to flow, depending on the value of IBETA. Table I lists all possible combinations where a value of 1 indicates the face is open to flow and a value of $O$ means the face is closed to flow. Negative values of IBETA are used in boundary cells.

In the program, no velocities or pressures are computed in obstacle cells, and all velocity components on faces of obstacle cells are set to zero automatically.

It also should be noted that because all velocity components within obstacles are set to zero, no-slip tangential velocity conditions are approximated at 
TABLE I

IBETA FLOW CONDITIONS

\begin{tabular}{lllll} 
IBETA & & $+x$ & $+y$ & $+z$ \\
\hline 0 or -8 & 0 & 0 & 0 \\
1 or -7 & 1 & 0 & 0 \\
2 or -6 & 0 & 1 & 0 \\
3 or -5 & 1 & 1 & 0 \\
4 or -4 & 0 & 0 & 1 \\
5 or -3 & 1 & 0 & 1 \\
6 or -2 & 0 & 1 & 1 \\
7 or -1 & 1 & 1 & 1
\end{tabular}

their surfaces but are only first-order accurate. That is, tangential velocities are zero at locations shifted into the obstacles one-half cell width from the actual boundary location.

\section{E. Numerical Stability Considerations}

Several restrictions on the time-step size must be observed to avoid numerical instabilities. If the user requests the AUTOT $=1.0$ condition in the input data, the code will automatically adjust the time step in subroutine DELTADJ to be as large as possible without violating the stability conditions. This routine also will reduce the time step when pressure iterations exceed a nominal value of 50 per cycle. Generally, the time step will float up or down with $2 \%$ changes per cycle unless a stability condition is violated, in which case a large reduction may occur. It is recommended that AUTOT $=1.0$ be used whenever possible to achieve optimum results.

If the user specifies AUTOT $=0.0$, which disables the automatic time-step control, then he must select time steps to satisfy the following criteria. First, fluid must not be permitted to flow across more than one computational cell in one time step. that is.

$$
\delta t<\min \left\{\delta x_{i} /|u|, \delta y_{j} /|v|, \delta z_{k} /|w|\right\} .
$$


This is clearly an accuracy condition because the advective fluxes are approximated by difference assumptions that only use information from neighboring cells. However, it is also a stability condition, which can be verified by performing a linear stability analysis on the advective portion of the momentum equation approximations. In fact, a review of worst-case situations indicates that the minimum value in the previous test [Eq. (39a)] should be multiplied by 0.25 , which allows a stability safety margin for most applications, that is,

$$
\delta t<0.25 \min \left\{\delta x_{i} /|u|, \delta y_{j} /|v|, \delta z_{k} /|w|\right\} .
$$

A linear analysis indicates that the time step also must be limited when a nonzero value of kinematic viscosity is used. This condition,

$v \delta t<0.5 / \max \left\{1 / \delta x_{i}^{2}, 1 / \delta y_{j}^{2}, 1 / \delta z_{k}^{2}\right\}$

is roughly the restriction that momentum not diffuse more than one mesh cell in one time step.

One more parameter. ALPHA, also must be set correctly to insure stability. This parameter controls the relative amounts of donor-cell and centered differencing used for the momentum advection terms. When ALPHA $=1.0 \mathrm{is} \mathrm{used,} \mathrm{the}$ above stability conditions are sufficient. Generally, one should always use an ALPHA value such that

$1.0 \geq A L P H A>\delta t \max \left\{|u| / \delta x_{i},|v| / \delta y_{j},|w| / \delta z_{k}\right\}$

For example, if Eq. (41) is satisfied, then we must have ALPHA $>0.25$. In no case should ALPHA exceed 1.0 .

\section{F. Graphics}

To interpret computational results, it is essential--particularly in threedimensional cases--to use computer graphic displays. Without some form of 
graphic presentation, the interpretation of the thousands of numerical values output by a typical calculation would be extremely difficult and time-consuming.

Three-dimensional calculations pose special difficulties associated with the two-dimensional nature of paper and film recording media. There are two possible solutions: graphic displays can be made of two-dimensional slices through a three-dimensional region, or perspective views of three-dimensional scenes can be drawn. Both types of displays have their uses, depending on the type of information to be displayed and the information sought. For this reason, SOLA-DM has been given capabilities for making two-dimensional and perspective view plots.

Two-dimensional plots can be requested for any coordinate plane by specifying the $1, J$, or $K$ index of the plane. For any such plane, the user can request velocity vector plots and/or pressure contour plots. Furthermore, the user can specify the 1 , J, or $k$ limits to be used in each plot. For instance, if a contour plot of pressure is wanted for only a portion of the $J=5 \mathrm{plane}$, the beginning and ending mesh indices in the $I$ and $K$ directions can be specified, and the plot will include only these cells.

The basic input for two-dimensional plots may be understood from the following example. To have three contour plots made, set the "number of contour plots" variable to 3 (NCPLTS $=3$ ). Then, for each plot, $k$, set the $1, J$, and $k$ cells to be included in the plot:

$$
\text { IC1 }(k), \quad I C 2(k), J C 1(k), J C 2(k), K C 1(k), K C 2(k),
$$

where (for example) $I C 1(k)$ is the beginning mesh index and $I C 2(k)$ is the ending mesh index in the $x$ direction. One pair of these numbers must have the same value, which indicates the plane to be plotted. There also must be as many sets of values as requested by NCPLTS. For each set, the user can specify the number of frame advances, NAC $(k)$. This value is defaulted to 1 , but sometimes it is useful to increase it when outputting to $35-\mathrm{mm}$ film so that extra film is available for mounting slides or making stereo slides. Similar input is used for two-dimensional velocity vector plots. A complete list of input variables is given below.

Perspective view plots can be made for velocities in selected planes, for marker particle distributions, and for the free surface configuration. The 
user may select several different eye locations for these plots. The input requirements for this consist of defining the number of eye locations wanted (NVEWS) and the coordinates of the eye for each location, for example, for the $k$ th eye point, [XEA $(k), Y E A(k)$, and $Z E A(k)]$. Another set of coordinates also can be set for each view $[X C A(k), Y C A(k)$, and $Z C A(k)]$. These points should be chosen to lie near the center of the region to be plotted. (They are defaulted to the origin- $-0,0,0$.) Their precise selection is not crucial. but they may alter the perspective significantly if they are not near the plot region. (See Ref. 7 for a more complete discussion.)

When the eye locations have been specified, perspective plots for velocity planes are requested by defining the $1, J$, and $K$ mesh limits for the vectors wanted (just as for two-dimensional plots) with the additional input of $\operatorname{IPERV}(k)=2$. This latter quantity is a flag that asks for a perspective plot. Normally IPERV $(k)$ is equal to 1 , which is its default value and the flag signaling a two-dimensional plot. When a perspective plot is requested, the user also must set the eye position wanted [IVVEW( $k)]$, which is set equal to the index $j$ of eye coordinate set XEA(j), and so on.

For particle plots, the entire mesh must be plotted. Thus, in this case, only the number of particle plots wanted (NPPLTS) is specified, and for each plot, the eye coordinate set wanted [IPVEW(k)] is selected. Also, the number of frame advances between plots, $\operatorname{NAP}(k)$, must be given if the default value of 1 is not acceptable. Contour plots cannot be shown in perspective; that is, the input parameter IPERC $(k)$ has no effect in the present version of the code.

Four additional input parameters in NAMELIST/XPUT/ are available to control graphic output. The parameter LPR should be set to zero (LPR = 0) when making movies as this will eliminate captions on all velocity and particle plots. Normally, LPR should be set equal to 2 , its default value, which gives captions and long prints on film. Setting LPR $=1$ will give plot captions, but no long prints on film.

The parameter IOBPL controls whether obstacles will be drawn in perspective view plots. Setting $10 B P L=1$ will add obstacles to these plots, whereas the default, ICBPL $=0$, will not. In summary, the following plot options are available.

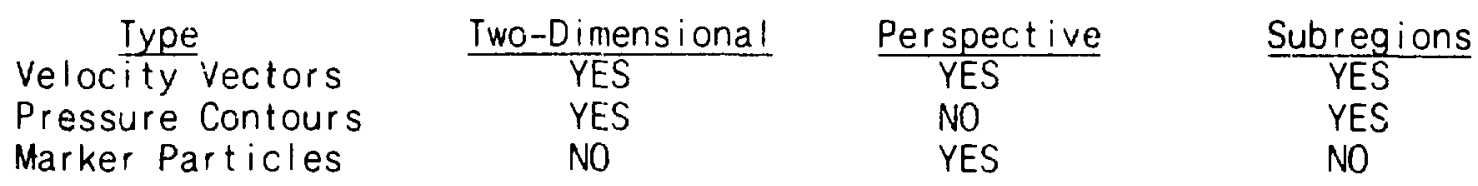




\section{RUNNING A PROBLEM}

Several things must be done to run a problem with SOLA-DM. First, you must specify the input data defining your problem and the output you want. Depending on the problem, you also may need to modify the code. After running a calculation, you will want to examine the printed and graphic outputs produced. If it is necessary to restart your calculation, only a few simple changes to two input files are needed. In this section, we describe the steps for modeling a problem and then illustrate with a sample calculation.

\section{A. Preparing an Input Data File}

Data for a problem are input by preparing an input file called INPUT. The first three lines of INPUT contain alphanumeric information describing the problem and must be input exactly as shown.

$\begin{array}{lll}\frac{\text { Line }}{1} & \frac{\text { Variable Name }}{\text { NAME (A80) }} & \text { Description } \\ 2 & \text { JNM (A10) } & \text { Lablem description } \\ 3 & \text { ILIST (3A10) } & \text { Special label }\end{array}$

The rest of the input is done with four NAMELIST groups: XPUT, MESHGN, GRAFIC, and PARTS. NAMELIST conventions and the variables in each group are described below.

1. NAMELIST Convention. The NAMELIST statement defines a list of variables or array names and associates that list of names with a unique group name. No format specification is necessary. The NAMELIST group name identifies the succeeding list of variables or array names. When a READ statement references a NAMELIST group name, input data are read from the designated file (INPUT). These NAMELIST values can be changed easily if new problem conditions are desired. (Note: Column one in each NAMELIST input line is ignored, so it should be left blank.) Each NAMELIST in the input file (INPUT) starts in column two with a $\$$ followed by the unique name of that NAMELIST. The variables contained in this record are separated by commas, and the NAMELIST is terminated with a $\$$ preceded by a space. In addition to its presence in the input file, the NAMELIST must appear in a statement at the beginning of the subroutine that contains its READ statement. 
For convenience, a complete list of input variables is given here for each of the NAMELIST blocks. Default values for each variable are given in the list along with a brief description. More details about each variable can be found in other sections of this report that deal with the appropriate topic. Where *** is listed in the default column, no default value has been set; these values must be input by the user for each problem. Subscripted variables are indicated with dummy indices.

2. Physical/Numerical/Control Data (NAMELIST/XPUT/). Basic problem information is entered in NAMELIST/XPUT/; all the variables are defined below. Give careful attention to the specifications for ALPHA (the amount of donor-cell differencing) and AUTODT (the time-step flag) described in Sec. IV.E. and the six boundary-type indicators (WL, WR, WF, WBK, WB, and WT) described in Sec. IV.D.1. The solution-type indicator (SOLATYPE) determines whether SOLA-DM will solve for fluid dynamics, particle transport, or both.

\section{NAMEL IST/XPUT/}

\begin{tabular}{|c|c|c|}
\hline Name & Default & Description \\
\hline ALPHA & 1.0 & $\begin{array}{l}\text { Controls weighting of upstream values in advection } \\
\text { approximations ( } 1.0 \text { for full upstream, } 0.0 \text { for central } \\
\text { differencing) }\end{array}$ \\
\hline AUTOT & 1.0 & $\begin{array}{l}\text { Automatic time-step flag ( } 1.0 \text { for automatic DELT, } 0.0 \\
\text { for constant DELT) }\end{array}$ \\
\hline CYL & 0.0 & $\begin{array}{l}\text { Geometry indicator }(0.0 \text { for plane coordinates, } 1.0 \text { for } \\
\text { cylindrical coordinates) }\end{array}$ \\
\hline DELTO & 0.02 & $\begin{array}{l}\text { Initial time step for fluid dynamics or particle } \\
\text { transport }\end{array}$ \\
\hline EPSI & 0.001 & Pressure iteration convergence criterion \\
\hline GX & 0.0 & Body acceleration in positive $x$ direction \\
\hline GY & 0.0 & Body acceleration in positive $y$ direction \\
\hline GZ & 0.0 & Body acceleration in positive $z$ direction \\
\hline 10BPL & 0 & $\begin{array}{l}\text { Obstacle plot indicator for perspective plots ( } 0 \text { for no } \\
\text { obstacle plot, } 1 \text { for plot) }\end{array}$ \\
\hline
\end{tabular}




\begin{tabular}{|c|c|c|}
\hline Name & Detault & Description \\
\hline IORDER & 1 & $\begin{array}{l}\text { Second-order accurate indicator (1 for first order, } 2 \\
\text { for second order) }\end{array}$ \\
\hline I TMAX & 20 & $\begin{array}{l}\text { Maximum number of pressure iterations allowed for each } \\
\text { time step. If only a steady-state solution is desired, } \\
\text { then setting ITMAX to a small number (for example, } 3 \text { or } \\
\text { 5) will decrease the computing time. }\end{array}$ \\
\hline IWALLS & $\star \star \star *$ & $\begin{array}{l}\text { Array of beginning and ending } I, J, K \text { mesh indices as- } \\
\text { sociated with walls normal to the } x \text { direction. Six num- } \\
\text { bers are required for each wall in the following order: } \\
I \text { (beginning). } J \text { (beginning), } K \text { (beginning), I (ending), } \\
J \text { (ending), and } K \text { (ending) }\end{array}$ \\
\hline JWALLS & 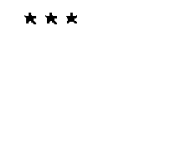 & $\begin{array}{l}\text { Array of beginning and ending } 1, J, K \text { mesh indices asso- } \\
\text { ciated with walls normal to the y direction. (See } \\
\text { IWALLS.) }\end{array}$ \\
\hline KWALLS & 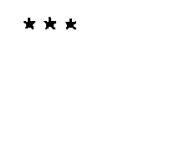 & $\begin{array}{l}\text { Array of beginning and ending } I, J, K \text { mesh indices asso- } \\
\text { ciated with walls normal to the } z \text { direction. (See } \\
\text { IWALLS.) }\end{array}$ \\
\hline LPR & 2 & $\begin{array}{l}\text { Film print and plot control ( } 0 \text { for full frame plots and } \\
\text { no captions, } 1 \text { for plots with captions, and } 2 \text { for prints } \\
\text { plus plots with captions) }\end{array}$ \\
\hline MAXCYC & 10 & Maximum number of time cycles allowed \\
\hline MOBS & $\star \star \star$ & $\begin{array}{l}\text { Array of beginning and ending } 1, J, K \text { mesh indices for } \\
\text { mesh obstacles. (See IWALLS.) }\end{array}$ \\
\hline NRSDUMP & -1 & $\begin{array}{l}\text { Tape dump read indicator ( }-1 \text { for no read; positive inte- } \\
\text { ger indicates restart tape dump number) }\end{array}$ \\
\hline NU & 0.0 & Coefficient of kinematic viscosity \\
\hline OMG & 1.7 & $\begin{array}{l}\text { Over-relaxation factor used in SOR pressure iteration } \\
\text { scheme }\end{array}$ \\
\hline PLTDT & 1.0 & Time interval between plots/prints output to film \\
\hline PRTDT & 1000.0 & Time interval between listings on paper \\
\hline REVPS & 0.0 & Rotation rate of mesh in revolutions per unit time \\
\hline SOLATYPE & 0.0 & $\begin{array}{l}\text { Solution type indicator ( } 0.0 \text { for fluid solution only, } \\
1.0 \text { for fluid and particle transport solutions, } 2.0 \text { for } \\
\text { particle transport only) }\end{array}$ \\
\hline TDDT & 10.0 & Time interval between tape dumps \\
\hline TWFIN & 10.0 & Problem time when calculation is finished \\
\hline
\end{tabular}




UI $\quad 0.0 \quad$ Initial fluid velocity in $x$ direction

VELMX $2.0 \quad$ Scaling factor for velocity vector plots (multiplies internally scaled vectors)

$V 1 \quad 0.0$

WB

1

WBK 1

WF

1

W1 $\quad 0.0$

WL $\quad 1$

WR $\quad 1$

WT $\quad 1$
Initial fluid velocity in y direction

Indicator for boundary condition at minimum z side (bottom) of mesh (1 for a rigid free-slip wall, 2 for a rigid no-sl ip wall, 3 for a continuative boundary, 4 for a periodic boundary in the $z$ direction, and 5 for a specified pressure boundary)

Indicator for boundary condition at maximum y side (back) of mesh (see WB)

Indicator for boundary condition at minimum y side (front) of mesh (see WB)

Initial fluid velocity in $z$ direction

Indicator for boundary condition at minimum $x$ side (left) of mesh (see WB)

Indicator for boundary condition at maximum $x$ side (right) of mesh (see WB)

Incicator for boundary conditions at maximum z side (top) of mesh (see WB)

3. Mesh Generation Data (NAMELIST/MESHGN/). A complete description of the computational mesh is given in NAMELIST/MESHGN/. The mesh is composed of an arbitrary number of submeshes as described in Sec. IV.C. A complete list of MESHGN variables follows.

$\begin{array}{lll}\text { Name } & \text { Default } & \text { Description } \\ \operatorname{DXMN(M)} & \star \star \star & x \text { direction cell size at } X C(M) \\ \operatorname{DYMN}(M) & * \star \star & y \text { direction cell size at } Y C(M) \\ \operatorname{DZMN}(M) & * \star * & Z \text { direction cell size at } Z C(M) \\ \operatorname{NKX} & * \star * & \text { Number of submesh data sets } M \text { in } x \text { direction }\end{array}$




\begin{tabular}{|c|c|c|}
\hline Name & Default & Description \\
\hline NKY & $\star \star \star *$ & Number of submesh data sets $M$ in y direction \\
\hline NKZ & $\star \star \star *$ & Number of submesh data sets $M$ in $z$ direction \\
\hline NXL (M) & $\star \star \star$ & Number of cells between $X L(M)$ and $X C(M)$ \\
\hline $\operatorname{NXR}(M)$ & $\star \star \star \star$ & Number of cells between $X C(M)$ and $X L(M+1)$ \\
\hline NYL $(M)$ & $\star \star \star$ & Number of cells between $Y L(M)$ and $Y C(M)$ \\
\hline $\operatorname{NYR}(M)$ & $\star \star \star$ & Number of cells between $Y C(M)$ and $Y L(M+1)$ \\
\hline NZL (ii ) & 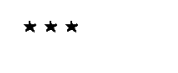 & Number of cells between $Z L(M)$ and $Z C(M)$ \\
\hline $\mathrm{NZR}(\mathrm{M})$ & $\star \star * *$ & Number of cells between $Z C(M)$ and $Z L(M+1)$ \\
\hline$X C(M)$ & 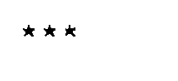 & $x$ coordinate of smallest cell location in submesh $M$ \\
\hline$X L(M)$ & 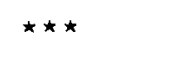 & $x$ coordinate of beginning of submesh $M$ \\
\hline$Y C(M)$ & $\star \star \star *$ & $y$ coordinate of smallest cell location in submesh $M$ \\
\hline$Y L(M)$ & 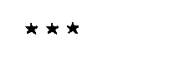 & $y$ coordinate of beginning of submesh $M$ \\
\hline$Z C(M)$ & $\star \star \star *$ & $z$ coordinate of smallest cell location in submesh $M$ \\
\hline$Z L(M)$ & $\star \star \star$ & $z$ coordinate of beginning of submesh $M$ \\
\hline
\end{tabular}

4. Graphic Output Data (NAMELIST/GRAFIC/). Velocity vector, contour, and particle plots are obtained by specifying the appropriate parameters in NAMELIST/ GRAFIC/. For each plot, you must specify the beginning and ending mesh indices in each of the three directions. If perspective views are desired, then sets of eye coordinates (XEA, YEA, ZEA) and object coordinates (XCA, YCA, ZCA) must be input. See Sec. IV.F for a more complete discussion of the graphics parameters. A complete list of GRAFIC variables separated by plot type is given below.

\section{----- NAMELIST/GRAFIC/ -...-- \\ PERSPECTIVE VIEW EYE POINTS}

$\begin{array}{lll}\text { Name } & \text { Default } & \text { Description } \\ \text { NVEWS } & 0 & \text { Number of view points } E \\ \text { XCA(E) } & 0.0 & \text { x coordinate of approximate mesh center }\end{array}$




$\begin{array}{lll}\text { XEA(E) } & 1.0 & \text { x coordinate of eye point for view } E \\ Y C A(E) & 0.0 & \text { y coordinate of approximate mesh center } \\ \text { YEA(E) } & 1.0 & \text { y coordinate of eye point for view } E \\ Z C A(E) & 0.0 & \text { z coordinate of approximate mesh center } \\ Z E A(E) & 1.0 & z \text { coordinate of eye point for view } E\end{array}$

CONTOUR PLOTS

$\begin{array}{lll}\text { Name } & \text { Default } & \text { Description } \\ \text { IPERC(C) } & 1 & \text { Not used } \\ \text { NAC(C) } & 0 & \text { Number of film frames advanced between contour plots } \\ \text { NCPLTS } & 10 & \text { Number of contour intervals to be drawn } \\ \text { NCONTUR } & 1 & \text { View point for contour plot C } \\ \text { ICVEW(C) } & 1 & \text { Minimum I index for plot C } \\ \text { IC1(C) } & \text { IM1 } & \text { Maximum I index for plot C } \\ \text { IC2(C) } & 1 & \text { Minimum J index for plot C } \\ \text { JC1(C) } & \text { JM1 } & \text { Maximum J index for plot C } \\ \text { JC2(C) } & 1 & \text { Minimum K index for plot C } \\ \text { KC1(C) } & \text { KM1 } & \text { Maximum K index for plot C } \\ \text { KC2(C) } & \end{array}$

\section{PARTICLE PLOTS}

Name Default

$\begin{array}{ll}\text { IPPKA(P) } & 1 \\ \text { IPVEW(P) } & 1 \\ \text { NAP(P) } & 1 \\ \text { NPPLTS } & 0\end{array}$

\section{Description}

Particle type for particle plot $P$

View point for particle plot $P$

Number of film frames advanced between particle plots Number of particle plots $P$ 
VELOCITY VECTOR PLOTS

\begin{tabular}{|c|c|c|}
\hline Name & Default & Description \\
\hline IINC & 1 & I direction cell increment between velocity vectors \\
\hline JINC & 1 & $J$ direction cell increment between velocity vectors \\
\hline KINC & 1 & $K$ direction cell increment between velocity vectors \\
\hline IPERV $(V)$ & 1 & $\begin{array}{l}\text { Indicator for type of plot ( } 1 \text { for two-dimensional slice } \\
2 \text { for perspective) }\end{array}$ \\
\hline $\operatorname{IVVEW}(V)$ & 1 & View point for perspective velocity vector plot $V$ \\
\hline IV1 $(V)$ & 1 & Minimum I index for plot $V$ \\
\hline IV2(V) & IM1 & Maximum I index for plot $V$ \\
\hline$J V 1(V)$ & 1 & Minimum $J$ index for plot $V$ \\
\hline $\mathrm{JV} 2(\mathrm{~V})$ & JM1 & Maximum $J$ index for plot $V$ \\
\hline $\mathrm{KV} 1(\mathrm{~V})$ & 1 & Minimum $K$ index for plot $V$ \\
\hline $\mathrm{KV} 2(\mathrm{~V})$ & KM1 & Maximum $K$ index for plot $V$ \\
\hline NAV $(V)$ & 1 & $\begin{array}{l}\text { Number of film frames advanced between velocity vector } \\
\text { plots }\end{array}$ \\
\hline NVPLTS & 0 & Number of velocity vector plots $V$ \\
\hline
\end{tabular}

5. Particle Input Data (NAMELIST/PARTS/). To use the discrete particle dynamics option, the user must input particle setup and control data in NAMELIST/PARTS/. A parallelepiped volume (block) of particles is placed in the fluid as follows.

(1) Define the left and right $x$ limits of the block with the variables XPL and XPR. The limits in the $y$ and $z$ directions are defined similarly by YPF, YPBK and ZPB, ZPT. If cylindrical coordinates are being used $(C Y L=1)$, YPF and YPBK have units of degrees. Particles will be placed at random spatial locations within the block. By defining a small volume, the user can approximate a point source of particles.

(2) Set the particle concentration (particles per unit volume) within the block with CPART and the time interval between particle inputs with PINPDT. The code will input CPART particles per unit volume into the block every PINPDT time units. 
(3) Define the ratio of fluid density to particle density (DENR), particle radius (SIZE), the laminar fluid kinematic viscosity (VISF), and the particle diffusion coefficient (NUP). Complete definitions of all particle input variables are given below.

The solution type indicator, SOLATYPE (set in NAMELIST/XPUT/), must be set to 1.0 or 2.0 to activate the particle dynamics option.

\begin{tabular}{|c|c|c|}
\hline Name & Default & Description \\
\hline $\operatorname{CD}(N)$ & 0.0 & Drag coefficient for particle type $N$ \\
\hline CPART & 0.0 & $\begin{array}{l}\text { Particle concentration (number of particles per unit } \\
\text { volume) in particle input volume }\end{array}$ \\
\hline IPPKT & 1 & $\begin{array}{l}\text { Number of initial rectangular particle blocks (only one } \\
\text { block permitted at this time) }\end{array}$ \\
\hline NI TERP & 5 & $\begin{array}{l}\text { Number of iterations for the Newton iteration cycle for } \\
\text { determining drag-induced particle velocity }\end{array}$ \\
\hline NPX & 0 & Not used \\
\hline NPY & 0 & Not used \\
\hline NPZ & 0 & Not used \\
\hline NUP & 0.0 & Diffusion coefficient for dispersing particles \\
\hline PINPDT & 1000 & Time interval between particle inputs \\
\hline $\operatorname{RATE}(\mathrm{N})$ & 0.0 & Not used \\
\hline VISF & 0.0 & $\begin{array}{l}\text { Coefficient of kinematic viscosity used in Stokes' drag } \\
\text { calculation }\end{array}$ \\
\hline $\operatorname{XPP}(N)$ & 0.0 & Not used \\
\hline$X P L$ & 0.0 & $\begin{array}{l}\text { Minimum } x \text { coordinate of initial rectangular particle } \\
\text { block }\end{array}$ \\
\hline XPR & 0.0 & $\begin{array}{l}\text { Maximum } \times \text { coordinate of initial rectangular particle } \\
\text { block }\end{array}$ \\
\hline YPBK & 0.0 & $\begin{array}{l}\text { Maximum y coordinate of initial rectangular particle } \\
\text { block }\end{array}$ \\
\hline
\end{tabular}




\begin{tabular}{|c|c|c|}
\hline Name & Default & Description \\
\hline YPF & 0.0 & $\begin{array}{l}\text { minimum y coordinate of initial rectangular particle } \\
\text { block }\end{array}$ \\
\hline $\operatorname{YPP}(N)$ & 0.0 & Not used \\
\hline ZPB & 0.0 & $\begin{array}{l}\text { Minimum } z \text { coordinate of initial rectangular particle } \\
\text { block }\end{array}$ \\
\hline $\mathrm{ZPP}(\mathrm{N})$ & 0.0 & Not used \\
\hline $\mathrm{ZPT}$ & 0.0 & $\begin{array}{l}\text { iviaximum z coordinate of initial rectangular particle } \\
\text { block }\end{array}$ \\
\hline
\end{tabular}

The dimensions of the subscripted variables used in the NAMELIST blocks may be changed by the user. These one-dimensional arrays all have length 10; that is,

$$
C=E=M=N=P=V=10
$$

\section{B. Code Modifications}

SOLA-DM is designed so that a variety of fluid dynamics problems can be modeled using data from the input file INPUT. However, you may need to modify the code to accommodate your problem. Most changes fall into the following categories.

1. Special Boundary Conditions. You may supplement or modify the existing boundary conditions (or obstacle/wall conditions) in subroutines BCSET and SETUP. For example, a time-dependent in-flow through a boundary or an internal sink are cases that require code modification.

2. Modifying the IBETA Array. Any boundary condition or internal flow condition (for example, obstacles and walls) not defined in the INPUT file will require you to modify the IBETA array for any affected cells. IBETA is a flag array that indicates the flow status for each cell. A discussion of IBETA and a list of all possible values is given in SeC. IV.D.2. Comment Cards in subroutine BETACAL indicate where changes to IBETA can be made.

3. Global Continuity. Because SOLA-DM is an incompressible flow code, the net in-flow into the problem domain must be balanced by ihe net out-flow; that is, 


$$
\int_{v} \underline{\nabla} \cdot \underline{u} d v=\underbrace{}_{S} \underline{u} \cdot \underline{n d A}=0 \text {. }
$$

where

$V \equiv$ the domain volume,

$S \equiv$ the domain surface.

$d v \equiv$ a differential volume element,

$d A \equiv$ a differential surface area, and

$\underline{n} \equiv$ the outward normal unit vector.

If you have modified the code so that fluid enters or leaves the domain, you should compute the volume flux (normal velocity times face area) for each affected cell face. At the end of subroutine BCSET, make sure that the variable FLUXIN is the sum of all such volume fluxes (A positive value for FLUXIN indicates a net flow into the domain).

4. Array Dimension Requirements. The maximum number of cells and particles that can be modeled is determined by the dimensioned size of arrays containing the primary fluid dynamic and particle transport variables. Six integers, which are defined below, may be changed in PARAMETER statements at the

\begin{tabular}{|c|c|c|}
\hline Variable & Description & Requir rement \\
\hline MIJ & $\begin{array}{l}\text { Defines the memory size } \\
\text { for } x-y \text { plane arrays }\end{array}$ & $M I J \geq I M A X * J M A X$ \\
\hline MK & $\begin{array}{l}\text { Defines the memory size } \\
\text { for z-direction arrays }\end{array}$ & MK $\geq \operatorname{KMAX}$ \\
\hline MS & $\begin{array}{l}\text { Defines the memory size } \\
\text { for mesh-wide arrays }\end{array}$ & $M S \geq I M A X * J M A X * K M A X$ \\
\hline MSP & $\begin{array}{l}\text { Defines the memory for } \\
\text { particle arrays }\end{array}$ & $\begin{array}{l}\text { MSP } \geq \text { the maximum number } \\
\text { of particles }\end{array}$ \\
\hline MS2 & Identical to MS & $M S 2=M S$ \\
\hline MSX & $\begin{array}{l}\text { Defines the memory size } \\
\text { for auxiliary arrays }\end{array}$ & $M S X \geq \operatorname{AMAXO}(M S, M S P)$ \\
\hline
\end{tabular}
beginning of the code to accommodate problems with more cells and/or particles. 
C. Output

Problem output is sent to the following five files.

MESHMAP This file contains a reference table of every mesh cell and Iists the absolute mesh index $(M)$, the logical grid indices $(i, j$, and $k$ ), the value of IBETA, the cell type (fluid, obstacle, or boundary), and which velocity components are allowed to take on nonzero values.

PLOT Graphics metafile containing plots at time intervals determined from the input variable PLTDT.

SOLAOUT Main printed output file containing (1) a list of input variables and (2) a list of $u, v, w, P$, and $\operatorname{div}(\underline{\nabla} \cdot \underline{u})$ for all cells at times determined from the input variable PRTDT.

TAPE6 Contains a listing of the values of all NAMELIST variables.

TAPE8 Binary dump file containing the primary problem arrays and control information at times determined by the input variable TDDT.

\section{Restarting a Problem}

Restarting a calculation is a simple two-step procedure.

(1) TAPE8 (the binary dump file) must be renamed RSSOLA (the restart dump file).

(2) Prepare the input file INPUT with the following information.

- The three problem identification cards (Cards 1--3)

- NRSDUMP; the restart dump number to be read from RSSOLA must be given in NAMELIST/XPUT/

- Any desired changes to variables in any NAMELIST group. If you wish to retain the value of NAMELIST variables used in the previous calculation, you need not reenter them.

Thus, the INPUT file for a problem restart requires a minimum of information. 
To help the new user get started, the SOLA-OM computer code described here has been set up to solve a simple problem. The results of our calculations for this problem are provided here and in Appendix $B$.

Computing the flow over a step is a convenient problem for testing the three-dimensional features of the code. The box-shaped computational domain has cartesian coordinates $0--3,0--1$, and $0--1$ in the $x, y$, and $z$ directions with a $0.3 \times 0.5 \times 0.5$ step (flow blockage) positioned in the $x=0, y=0$, $z=0$ corner. The domain is modeled by a $10 \times 10 \times 10$ mesh for a total of 1000 cells. The INPUT file and output file (SOLAOUT) for this problem are given in Appendix B.

Flow enters the domain at the left $(x=0)$ boundary with a velocity of $u=1000$, flows over the step, and exits the domain at the right $(x=3)$ boundary. The boundary condition indicator for the left boundary, WL, is set to zero in the INPUT file, which means that the code will not set velocities and pressures at the left boundary automatically. We model the in-flow with the changes shown in the code modification file. (See Appendix B. Sec. 11.) The top and bottom boundaries $(z=0, z=1)$ and the two side boundaries $(y=0, y=1)$ are modeled as free-slip walls. The kinematic viscosity is $\nu=0.15$, which means that the Reynolds number based on the height of the step is

$$
\operatorname{Re}=\frac{u h}{v}=\frac{(1000)(0.5)}{0.15}=3333.3
$$

The calculation reaches a steady state after approximately 2200 time steps at a time of $0.4 \mathrm{~s}$. Figures $6(a)$ and $\hat{b}(b)$ show velocity fields at $J=2$ (an $x-z$ plane) and $K=4$ (an $x-y$ plane) at $t=0.4 \mathrm{~s}$. A recirculation pattern in the wake of the step is clearly evident. A perspective view [Fig. 6(c)] shows the velocity vectors in the $J=2 p$ lane and for the lowest horizontal plane $(K=2)$.

Introducing particles into this flow requires a restart INPUT file. In addition to the particle specifications listed in NAMELIST/PARTS/, we set NRSDUMP $=1$ (to read the first data dump on file RSSOLA) and SOLATYPE $=2$ (to compute only the motion of particles, that is, the fluid flow field is "frozen") in NAMELIST/XPUT/. A listing of this restart INPUT file is given in Appendix B. 
In this example, 100 particles are placed at random locations in the parallelelepiped volume defined by $x=0$ to $0.25, y=0.25$ to 0.75 , and $z=0.50$ to 0.75 [Fig. $7(\mathrm{a})$ ]. The locations of the particles after $1.5 \times 10^{-3}$ and $3 \times 10^{-3} \mathrm{~s}$ are shown in Figs. $7(b)$ and $7(c)$. In the last figure, 25 particles have left the domain, and the remaining particles have spread out as a result of the nonuniform fluid velocity in the wake of the step.

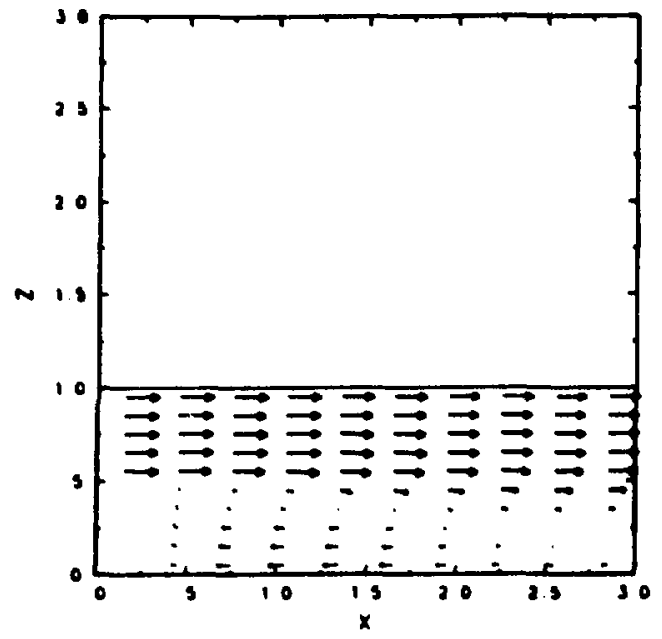

(a) $J=2, x-z$ plane.

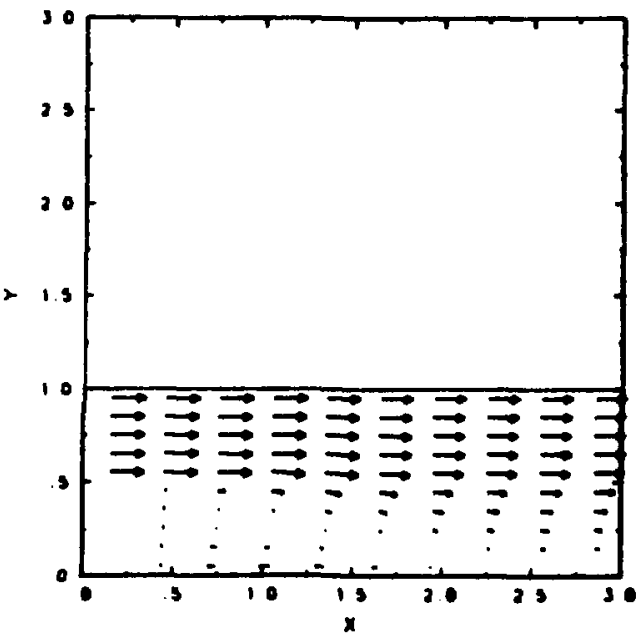

(b) $K=4, x-y$ plane.

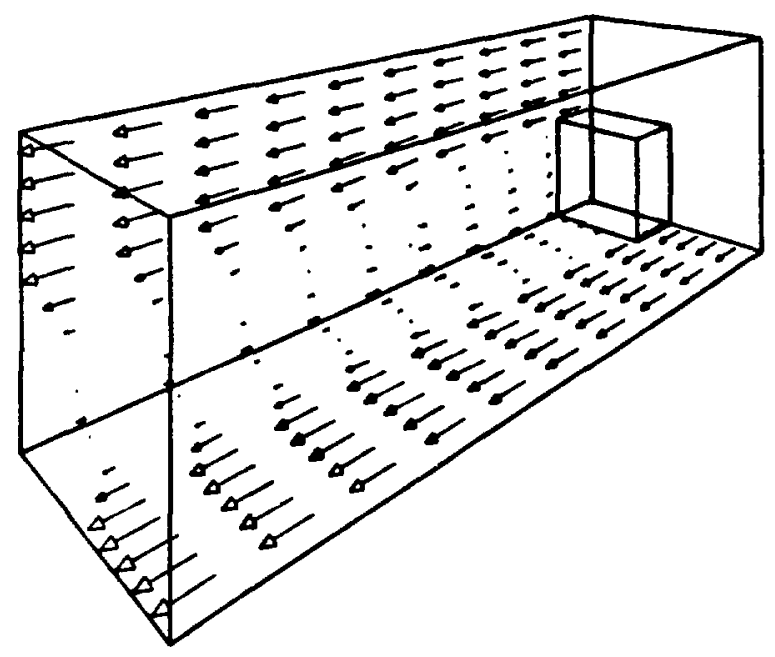

(c) Perspective view showing vectors in the $J=2$ and $K=2$ planes.

Fig. 6 .

Steady-state $(\underline{t}=0.4)$ velocity fields for flow over a step. 


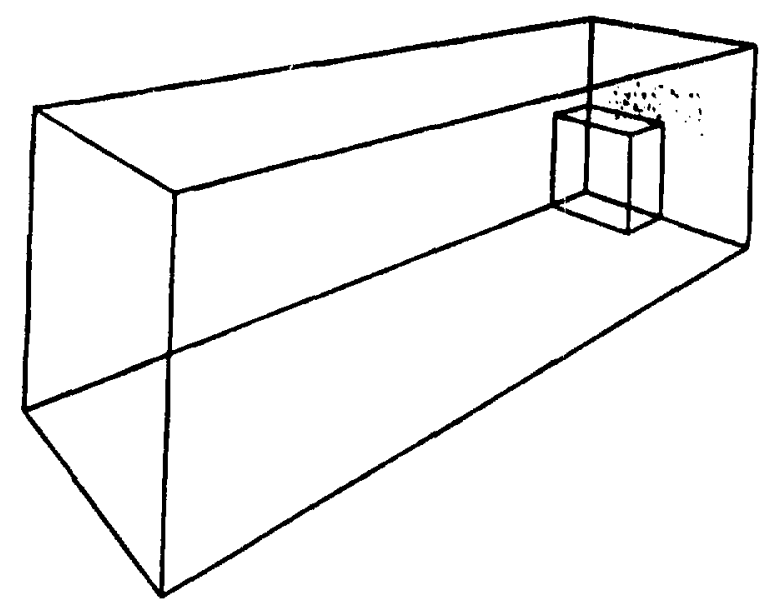

(a) Time $=0.4$. Particles in initial configuration

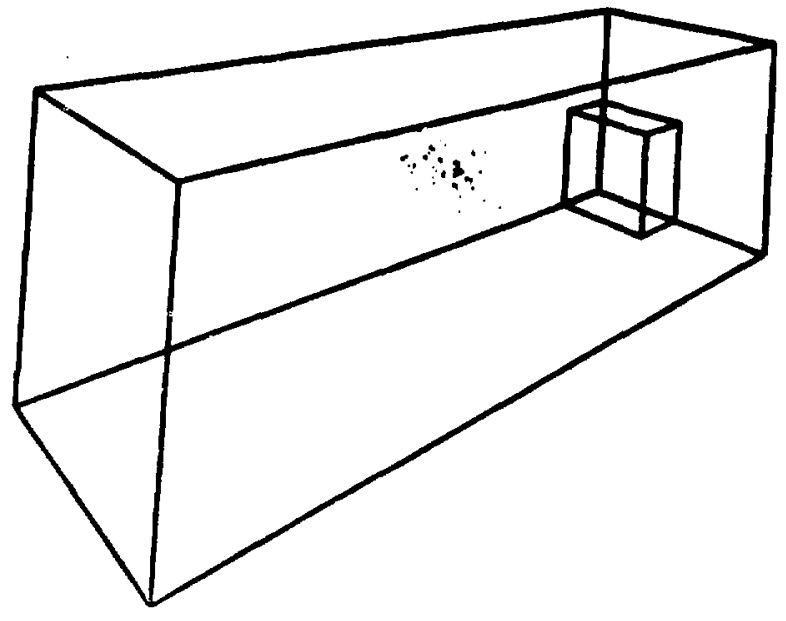

(b) Time $=0.4015$.

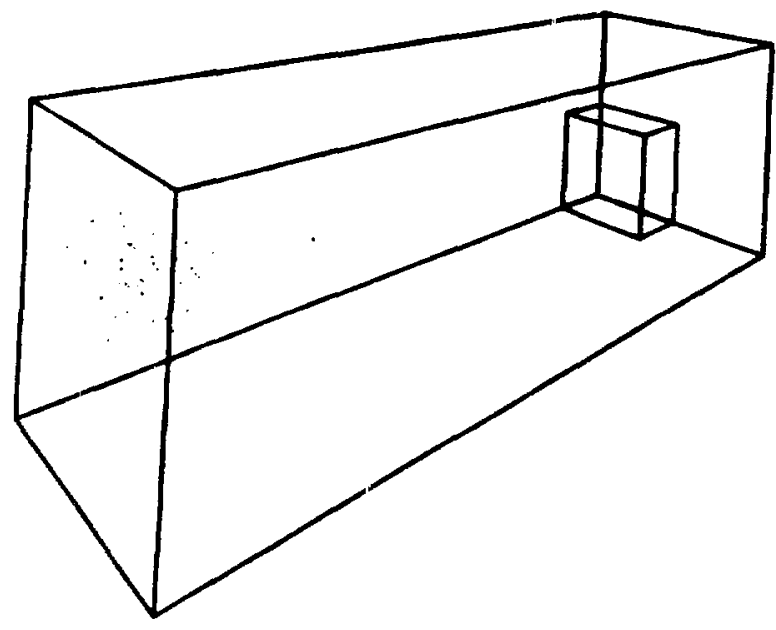

(c) Time $=0.4030$.

Fig. 7.

Transport of particles in the steady-state flow field. 


\section{REFERENCES}

1. F. H. Harlow and J. E. Welch, "Numerical Calculation of Time-Dependent Viscous Incompressible Flow," Phys. Fluids $\underline{8}, 2182$ (1965).

2. J. E. Welch, F. H. Harlow, J. P. Shannon, and B. J. Daly, "The MAC Method: A Computing Technique for Solving Viscous, Incompressible, Transient FluidFlow Problems Involving Free Surfaces." Los Alamos Scientific Laboratory report LA-3425 (1966).

3. C. W. Hirt, B. D. Nichols, and N. C. Romero, "SOLA-A Numer ical Solut ion Algorithm for Transient Fluid Flows," Los Alamos Scientific Laboratory report LA-5852 (1975).

4. C. W. Hirt and B. D. Nichols, "Volume of Fluid (VOF) Method for the Dynamics of Free Boundaries," J. Comp. Phys. 39, 201 (1981).

5. B. J. Daly and M. D. Torrey, "SOLA-PTS: A Transient, Three-Dimensional Algorithm for Fluid-Thermal Mixing and Wall Heat Transfer in Complex Geometries," Los Alamos National Laboratory report LA-10132-MS (1984).

6. R. Chandra, "Conjugate Gradient Methods for Partial Differential Equations," Ph.D. Thesis, Yale University (1978).

7. C. W. Hirt and J. L. Cook, "Perspective Displays for Three-Dimensional Finite Difference Calculations," Computers and Fluids 3, 293 (1974). 
SOLA-DM uses a number of system routines to obtain graphic output on film or a CRT. Graphic output is obtained by using two Los Alamos graphics libraries, CGS and CGSHIGH. CGS (Common Graphics System) is a library of subroutines that provide graphics primitives, and CGSHIGH is a library of high-level graphics subroutines based on CGS. Brief descriptions of the graphics subroutines used by SOLA-DM are given below.

\section{CGS Subroutine Calls}

CALL GLINA2 $(X, Y)$ draws a line from the current position to cartesian coordinates $(X, Y)$ in window space.

CALL GMOVA2 $(X, Y)$ moves to the cartesian coordinates $(X, Y)$ in window space.

CALL GMRKA2 $(X, Y)$ plots a single point at the coordinate $(X, Y)$ in window space.

CALL GPAGE advances the film one frame or erases the screen on a graphics terminal.

CALL GPALET $(N)$ selects a color. The color selected goes from red to green to blue to red as $\mathrm{N}$ goes from 1 to 60 . For other values of $N$, the color is white.

CALL GPLOT (ICL, ITITLE, NCHAR) initializes CGS. ICL is the classification identifier expressed as a single-character Hollerith string (for example. 1HU), ITITLE is a Hollerith title and NCHAR is the number of characters in ITITLE.

CALL GTEXT (ITEXT, NCHAR) displays the Hollerith text string ITEXT where NCHAR is the number of characters in ITEXT.

CALL GTFNTA selects the stroke mode and font to be used to display text strings using GTEXT.

CALL GTSTRM selects the string mode to display a text string using GTEXT.

CALL GTWDTH specifies the character width at which software-generated stroke-mode text strings will be displayed.

CALL GVIEW2 (VXBEG, VXEND, VYBEG, VYEND) defines a viewport on the view surface (screen). VXBEG and VXEND are the beginning and ending $x$ values of the viewport, and VYBEG and VYEND are the corresponding $y$ values. 
CALL GWIDTH (FWIDTH) sets the line width attribute for lines and text.

CALL. GWIND2 (WXMIN, WXMAX, WYMIN, WYMAX) defines a window in the world (or user) coordinate system. WXMIN and WXMAX are the minimum and maximum $x$ values of the window, and WYMIN and WYMAX are the corresponding $y$ values.

\section{CGSHIGH Subroutine Calls}

(NOTE: Calling arguments have been omitted for CGSHIGH subrout ines.)

CALL HAXRUL controls the ruling of $x$ and $y$ axes.

CALL HAXSCL computes the axis scale numbers from the data.

CALL HCVLS sets the curve line style (solid, dot, dash, and so on).

CALL HHU2DU converts coordinates from CGSHIGH units to user data units.

CALL HSEQV sets the CGSHIGH space aspect ratio equal to the CGS viewport aspect ratio.

CALL HXY draws an $x-y$ plot with a grid system and axis labels.

\section{Miscellaneous System Calls}

CALL LIBHI initializes CGSHIGH graphics.

CALL DATEH (DAY) returns the date as a character string of the form $M M / D D / Y Y$.

CALL TIMEH (CLOCK) returns the time of day as a character string of the form HH:MM:SS.

CALL CPUTIME (TIME) returns the CPU time used by the job up to this point.

CALL EXITA (N) terminates the code. Each call to EXITA in SOLA-DM has a different value of $N$, which is retained by the operating system and available for determining the cause of the exit, if necessary. 
INPUT AND OUTPUT FILES FOR SAMPLE PROBLEM

1. INPUT FILE "INPUT"

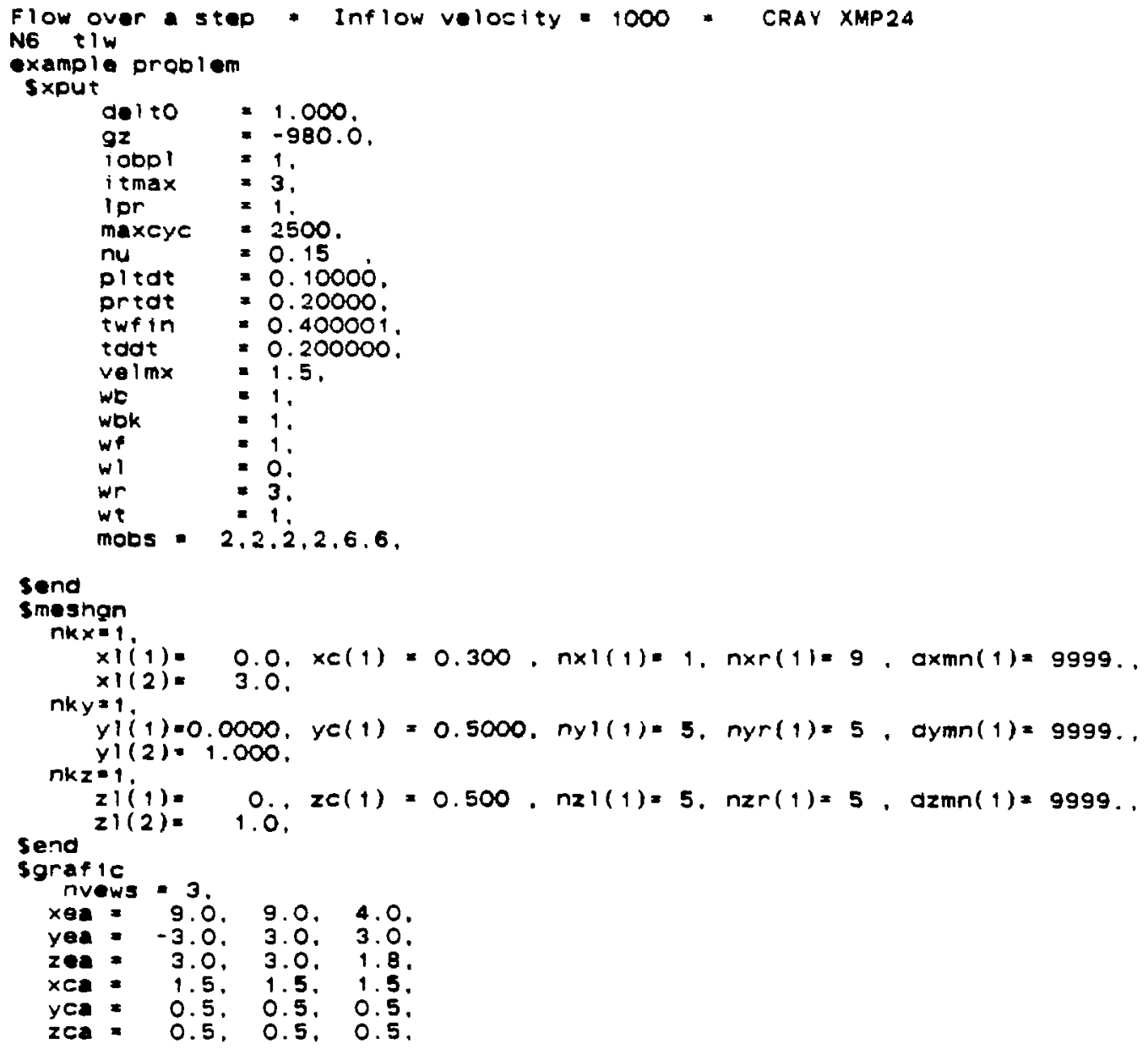

nvpl ts=8, i ince=1, jinc=1, kinc=1.

ivvew $(1)=1$.

$i v 1(1)=6, i \vee 2(1)=6, j \vee 1(1)=1, j \vee 2(1)=11, \quad k \vee 1(1)=1, k \vee 2(1)=11$. $\operatorname{nav}(1)=1, \quad$ iperv $(1)=1$.

I vvew $(2)=1$.

$i \vee 1(2)=1, i \vee 2(2)=11, j \vee 1(2)=6, j \vee 2(2)=6, \quad k \vee 1(2)=1, k \vee 2(2)=11$. $\operatorname{nav}(2)=1$, iperv $(2)=1$,

ibvew $(3)=1$.

ivi $(3)=1, i \vee 2(3)=11, j \vee 1(3)=1, j \vee 2(3)=11, \quad k \vee 1(3)=4, k \vee 2(3)=4$.

$\operatorname{nav}(3)=1, i \operatorname{perv}(3)=1$.

trvew $(4)=1$.

$i \vee 1(4)=1, i \vee 2(4)=11, j \vee 1(4)=6, j \vee 2(4)=6, \quad k \vee 1(4)=1, k \vee 2(4)=11$. $\operatorname{nav}(4)=1, i \operatorname{perv}(4)=2$. 


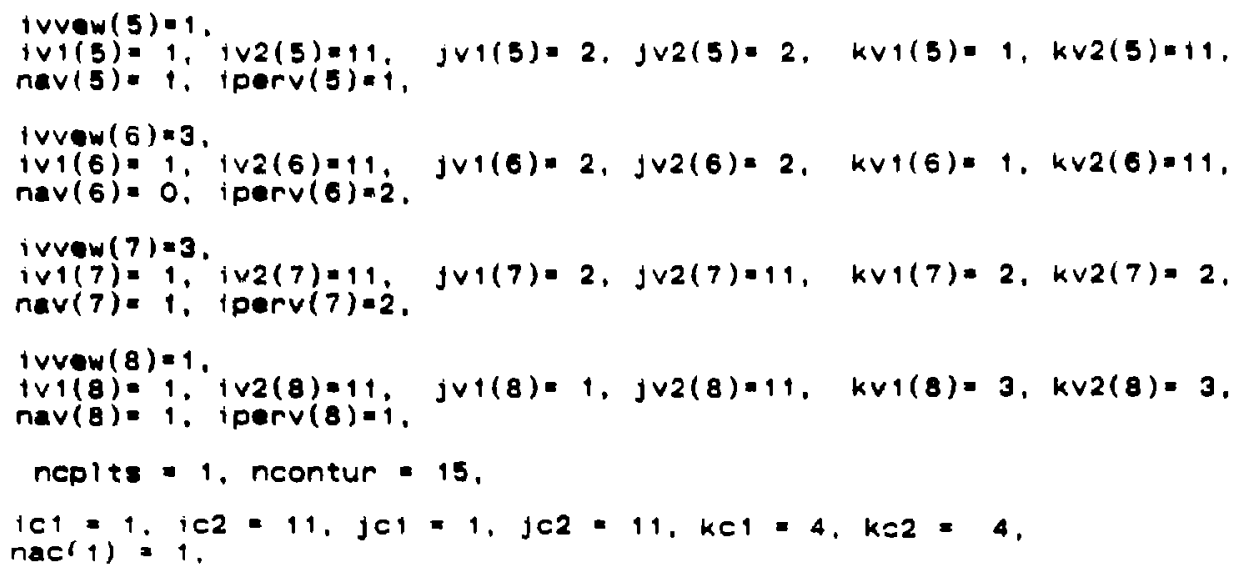

\section{CODE MODIFICATION FILE}

Each I ine in SOLA-DM is identified by a HISTORIAN statement number in columns 73 through 80 . Statement numbers consist of a deck name (usually the subrout ine name) and a number. The code modifications given below are written as HISTORIAN directives. A line of the form *I, deckname.n means the FORTRAN statement(s) that appear between the *I line and the next line beginning with a * are inserted following the line deckname. $n$. The line ${ }^{\star} D$, deckname.m, $n$ means to delete statements deckname.m through deckname.n and replace them with any lines between the ${ }^{\star} D$ and the next ${ }^{*} l$ ine.

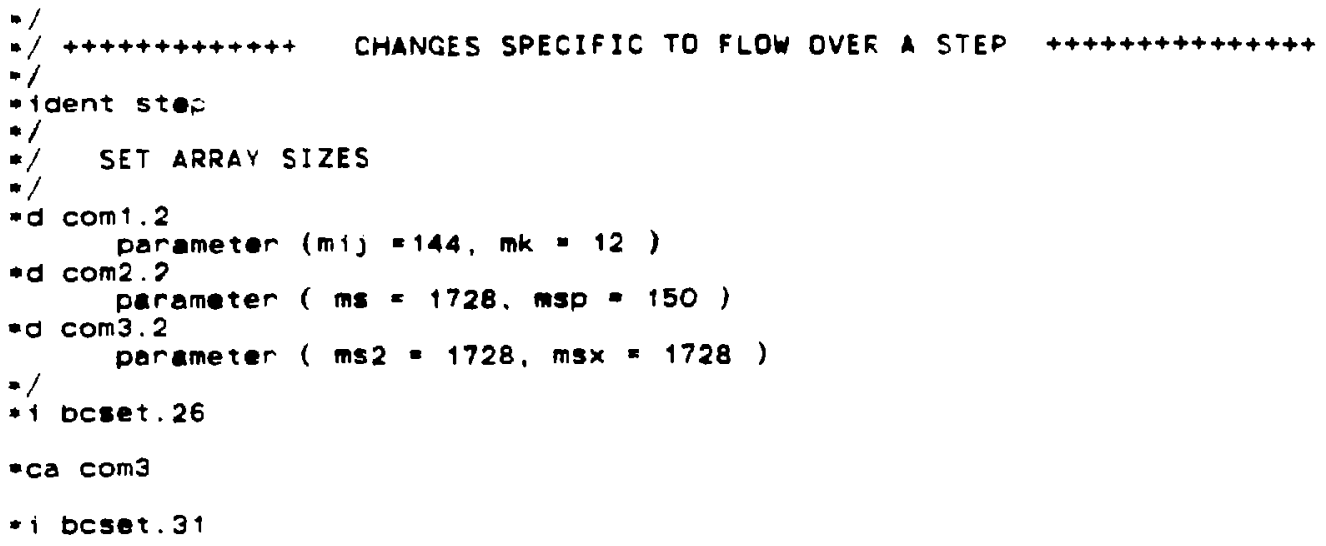




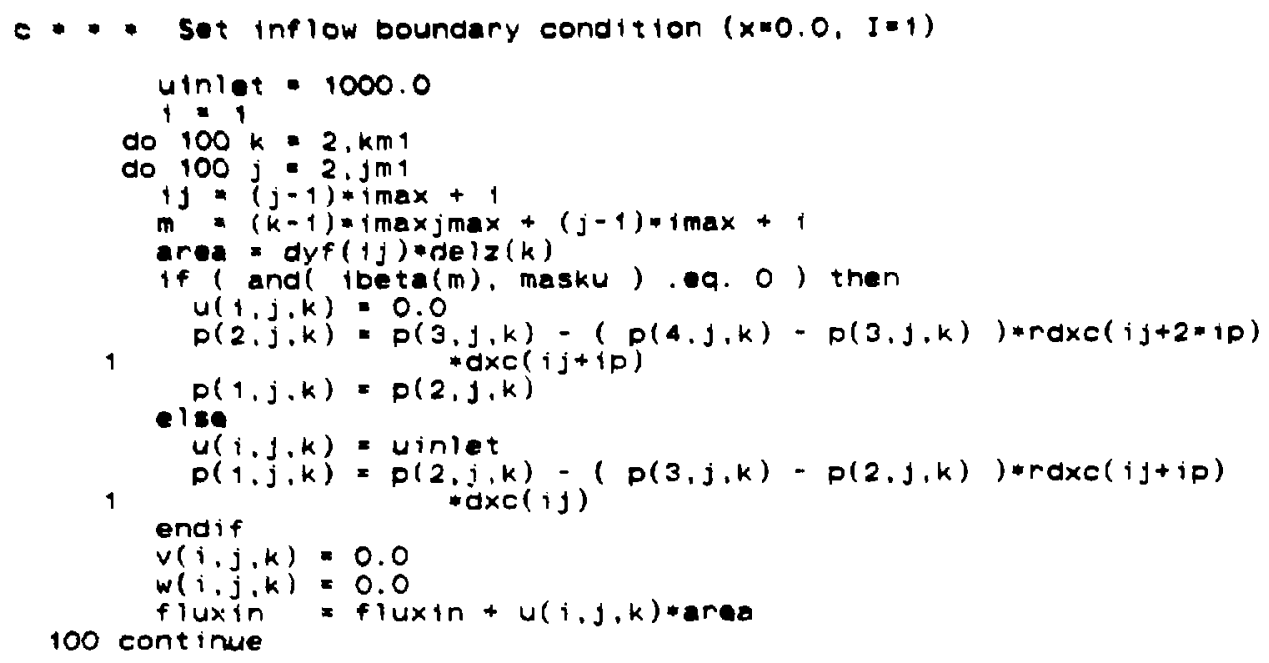

\section{OUTPUT FILE "SOLAOUT"}

A portion of the SOLAOUT file at the problem end time $(0.4 \mathrm{~s})$ is listed on the following pages. For each cell in the mesh, the following information is written in tabular form.

$\begin{array}{ll}M & \text { Absolute mesh cell index } \\ K & \text { Mesh index in the } z \text { direction } \\ J & \text { Mesh index in the } y \text { direction } \\ I & \text { Mesh index in the } x \text { direction } \\ u & \text { Fluid velocity in the } x \text { direction } \\ v & \text { Fluid velocity in the } y \text { direction } \\ w & \text { Fluid velocity in the } z \text { direction } \\ P & \text { Pressure/density ( } / \rho) \\ \text { div } & \text { Divergence of the velocity field }(\underline{\nabla}, \underline{u})\end{array}$


Flow over step - Inflow velocity. 1000. CRAY XMP24 10/30/87 09:05:41 iter: 1 time=4.001190-01

\begin{tabular}{|c|c|c|c|c|c|c|c|c|}
\hline 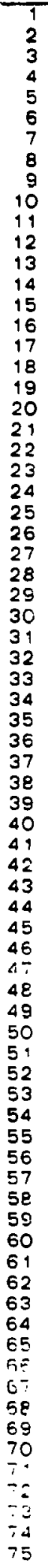 & 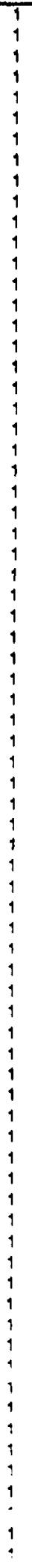 & 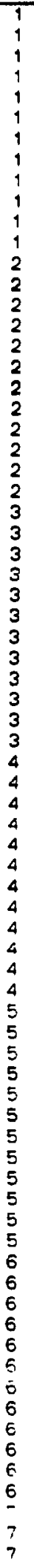 & 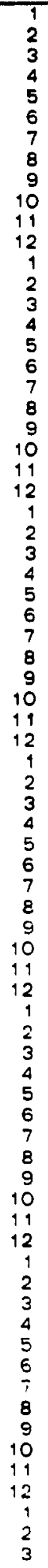 & 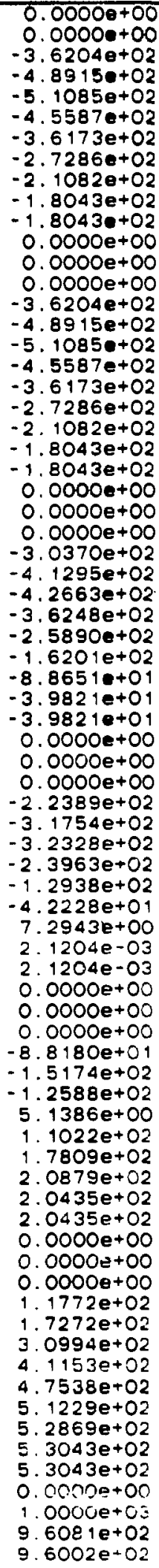 & 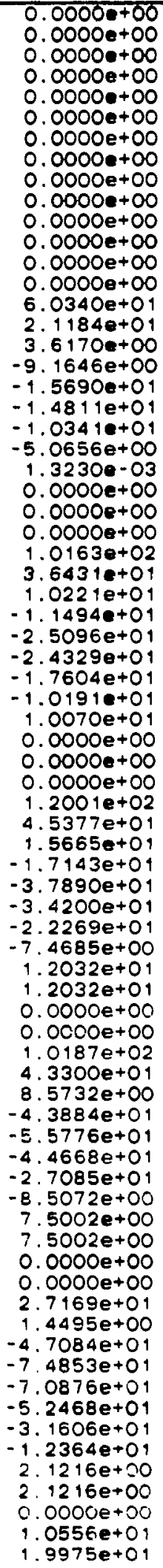 & $\begin{array}{l}0.0000 e+00 \\
0.0000 e+00 \\
0.0000++00 \\
0.0000 e+00 \\
0.0000++00 \\
0.0000 e+00 \\
0.0000 e+00 \\
0.0000 e+00 \\
0.0000 e+00 \\
0.0000 e+00 \\
0.0000 e+00 \\
0.0000 e+00 \\
0.0000 e+00 \\
0.0000 e+00 \\
0.0000 e+00 \\
0.0000 e+00 \\
0.0000 e+00 \\
0.0000 e+00 \\
0.0000 e+00 \\
0.0000 e+00 \\
0.0000 e+00 \\
0.0000 e+00 \\
0.0000 e+00 \\
0.0000 e+00 \\
0.0000 e+00 \\
0.0000 e+00 \\
0.0000 e+00 \\
0.0000 e+00 \\
0.0000 e+00 \\
0.0000 e+00 \\
0.0000 e+00 \\
0.0000 e+00 \\
0.0000 e+00 \\
0.0000 e+00 \\
0.0000 e+00 \\
0.0000 e+00 \\
0.0000 e+00 \\
0.0000 e+00 \\
0.0000 e+00 \\
0.0000 e+00 \\
0.0000 e+00 \\
0.0000 e+00 \\
0.0000 e+00 \\
0.0000 e+00 \\
0.0000 e+00 \\
0.0000 e+00 \\
0.0000 e+00 \\
0.0000 e+00 \\
0.0000 e+00 \\
0.0000 e+00 \\
0.0000 e+00 \\
0.0000 e+00 \\
0.0000 e+00 \\
0.0000 e+00 \\
0.0000 e+00 \\
0.0000 e+00 \\
0.0000 e+00 \\
0.0000 e+00 \\
0.0000 e+00 \\
0.0000 e+00 \\
0.0000 e+00 \\
0.0000 e+00 \\
0.0000 e+00 \\
0.0000 e+00 \\
0.0000 e+00 \\
0.0000 e+00 \\
0.0000 e+00 \\
0.0000 e+00 \\
0.0000 e+00 \\
0.0000 e+00 \\
0.0000 e+00 \\
0.0000 e+00 \\
0.0000 e+00 \\
0.0000 e+00 \\
0.0000 e+00\end{array}$ & $\begin{array}{l}1.0874 e+05 \\
1.0871 e+05 \\
6.9225 e+04 \\
2.0740++04 \\
1.0088 e+04 \\
3.8748 e+04 \\
8.5097 e+04 \\
1.2255 e+05 \\
1.4463 e+05 \\
1.5498 e+05 \\
1.5748 e+05 \\
1.5978 e+05 \\
1.0874 e+05 \\
1.0874 e+05 \\
6.9323 e+04 \\
2.0838 e+04 \\
1.0186 e+04 \\
3.8846 e+04 \\
8.5195 e+04 \\
1.2265 e+05 \\
1.4473 e+05 \\
1.5508 e+05 \\
1.5758 e+05 \\
1.5978 e+05 \\
9.9773 e+04 \\
9.9773 e+04 \\
6.2948 e+04 \\
1.8217 e+04 \\
8.2619 e+03 \\
3.7672 e+04 \\
8.5087 e+04 \\
1.2295 e+05 \\
1.4505 e+05 \\
1.5534 e+05 \\
1.5747 e+05 \\
1.5956 e+05 \\
8.8824 e+04 \\
8.8824 e+04 \\
5.4334 e+04 \\
1.4455 e+04 \\
5.0339 e+03 \\
3.5314 e+04 \\
8.4481 e+04 \\
1.2296 e+05 \\
1.4523 e+05 \\
1.5589 e+05 \\
1.5723 e+05 \\
1.5881 e+05 \\
8.2692 e+04 \\
8.2692 e+04 \\
4.7825 e+04 \\
1.0887 e+04 \\
1.2086 e+03 \\
3.2747 e+04 \\
8.3372 e+04 \\
1.2260 e+05 \\
1.4509 e+05 \\
1.5576 e+05 \\
1.5676 e+05 \\
1.5790 e+05 \\
8.5353 e+04 \\
8.5353 e+04 \\
4.9049 e+04 \\
9.8797 e+03 \\
5.0233 e+02 \\
3.2687 e+04 \\
8.3271 e+04 \\
1.2121 e+05 \\
1.4308 e+05 \\
1.5357 e+05 \\
1.5502 e+05 \\
1.5634 e+05 \\
6.1089 e+04 \\
5.4037 e+04 \\
4.6214 e+04\end{array}$ & 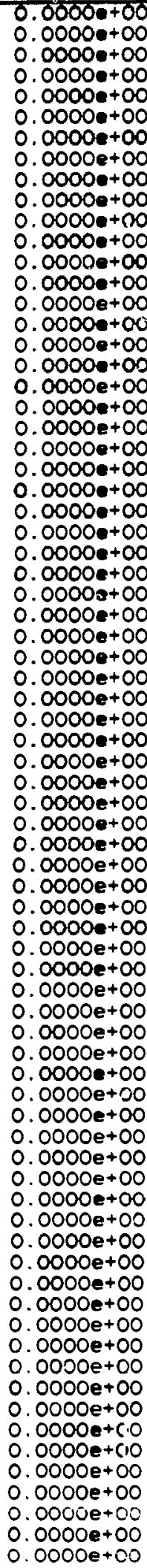 \\
\hline
\end{tabular}


Flow over step * Inflow velocity - 1000. CRAY XMP24 10/30/87 09:05:41 iter: 1 timex 4.001190-01 cyclea

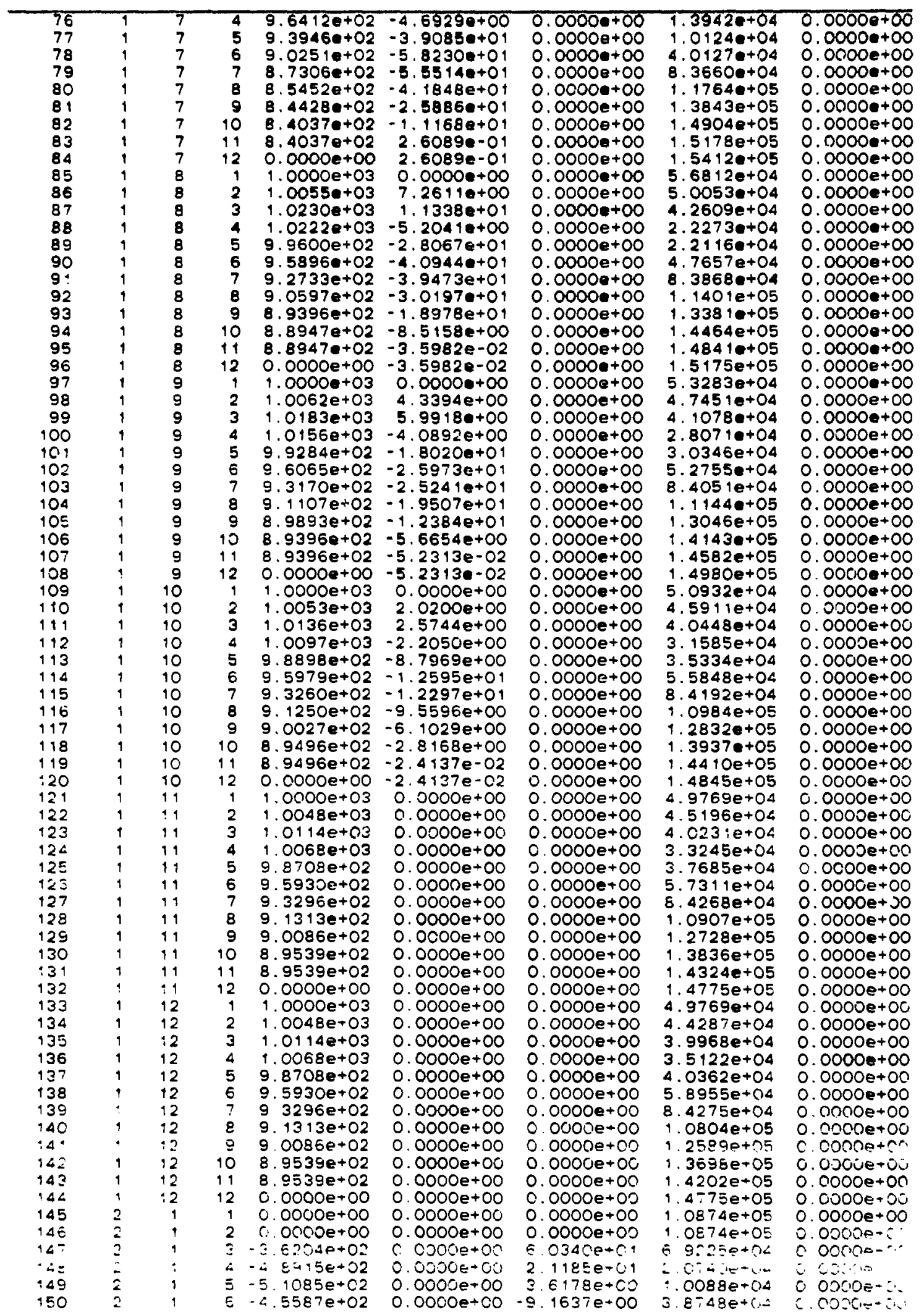


Flow over a step * Inflow velocity a 1000 . CRAY XMP24 10/30/87 Og:05:41 iter: i time=4.001190-01 cyclen 2216
M $\checkmark \quad I$ $u$ $\checkmark$ $w$

\begin{tabular}{|c|c|c|c|c|c|c|c|c|}
\hline 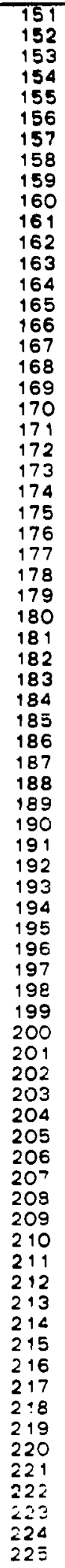 & $\begin{array}{l}9 \\
2 \\
2 \\
2 \\
2 \\
2 \\
2 \\
2 \\
2 \\
2 \\
2 \\
2 \\
2 \\
2 \\
2 \\
2\end{array}$ & 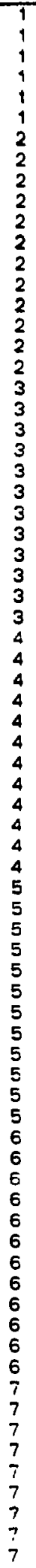 & 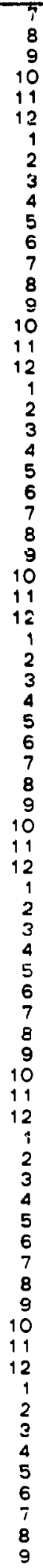 & $\begin{array}{r}-3.6173 e+02 \\
-2.7286 e+02 \\
-2.1082 e+02 \\
-1.8043 e+02 \\
-1.8043 e+02 \\
0.0000 e+00 \\
0.0000 e+00 \\
0.0000 e+00 \\
-3.6204 e+02 \\
-4.8915 e+02 \\
-5.1085 e+02 \\
-4.5587 e+02 \\
-3.6173 e+02 \\
-2.7286 e+02 \\
-2.1082++02 \\
-1.8043 e+02 \\
-1.8043 e+02 \\
0.0000 e+00 \\
0.0000 e+00 \\
0.0000 e+00 \\
-3.0370 e+02 \\
-4.1295 e+02 \\
-4.2663 e+02 \\
-3.6248 e+02 \\
-2.5890 e+02 \\
-1.6201 e+02 \\
-8.8651 e+01 \\
-3.9821 e+01 \\
-3.9821 e+01 \\
0.0000 e+00 \\
0.0000 e+00 \\
0.0000 e+00 \\
-2.2389 e+02 \\
-3.1754 e+02 \\
-3.2328 e+02 \\
-2.3963 e+02 \\
-1.2938 e+02 \\
-4.2228 e+01 \\
7.2943 e+00 \\
2.1204 e-03 \\
2.1203 e-03 \\
0.0000 e+00 \\
0.0000 e+00 \\
0.0000 e+00 \\
-8.8180 e+01 \\
-1.5174 e+02 \\
-1.2588 e+02 \\
5.1386 e+00 \\
1.1022 e+02 \\
1.7809 e+02 \\
2.0879 e+02 \\
2.0435 e+02 \\
2.0435 e+02 \\
0.0000 e+00 \\
0.0000 e+00 \\
0.0000 e+00 \\
1.1772 e+02 \\
1.7272 e+02 \\
3.0994 e+02 \\
4.1153 e+02 \\
4.7538 e+02 \\
5.1229 e+02 \\
5.2869 e+02 \\
5.3043 e+02 \\
5.3043 e+02 \\
0.0000 e+00 \\
1.0000 e+03 \\
9.6081 e+02 \\
9.6002 e+02 \\
9.6412 e+02 \\
9.3946 e+02 \\
9.0251 e+02 \\
8.7305 e+02 \\
8.5452 e+02 \\
8.4428 e+02\end{array}$ & $\begin{array}{r}0.0000 e+00 \\
0.0000 e+00 \\
0.0000 e+00 \\
0.0000 e+00 \\
0.0000 e+00 \\
0.0000 e+00 \\
0.0000 e+00 \\
0.0000 e+00 \\
6.0340 e+01 \\
2.1184 e+01 \\
3.6170 e+00 \\
-9.1646 e+00 \\
-1.5690 e+01 \\
-1.4811 e+01 \\
-1.0341 e+01 \\
-5.0656 e+00 \\
1.3230 e-03 \\
0.0000 e+00 \\
0.0000 e+00 \\
0.0000 e+00 \\
1.0163 e+02 \\
3.6431 e+01 \\
1.0221 e+01 \\
-1.1494 e+01 \\
-2.5096 e+01 \\
-2.4329 e+01 \\
-1.7604 e+01 \\
-1.0191 e+01 \\
1.0070 e+01 \\
0.0000 e+00 \\
0.0000 e+00 \\
0.0000 e+00 \\
1.2001 e+02 \\
4.5377 e+01 \\
1.5665 e+01 \\
-1.7443 e+01 \\
-3.7890 e+01 \\
-3.4200 e+01 \\
-2.2269 e+01 \\
-7.4685 e+00 \\
1.2032 e+01 \\
1.2032 e+01 \\
0.0000 e+00 \\
0.0000 e+00 \\
1.0187 e+02 \\
4.3300 e+01 \\
8.5732 e+00 \\
-4.3884 e+01 \\
-5.5776 e+01 \\
-4.4668 e+01 \\
-2.7085 e+01 \\
-8.5072 e+00 \\
7.5002 e+00 \\
7.5002 e+00 \\
0.0000 e+00 \\
0.0000 e+00 \\
2.7169 e+01 \\
1.4495 e+00 \\
-7.7084 e+01 \\
-7.4853 e+01 \\
-5.24680+1606 e+01 \\
-1.2364 e+01 \\
2.1216 e+00 \\
2.1216 e+00 \\
0.0000 e+00 \\
1.0556 e+01 \\
1.9975 e+01 \\
-4.6929 e+00 \\
-3.9085 e+01 \\
-5.2230 e+01 \\
-5.5514 e+01 \\
-4.1848 e+01 \\
-2.5886 e+01\end{array}$ & 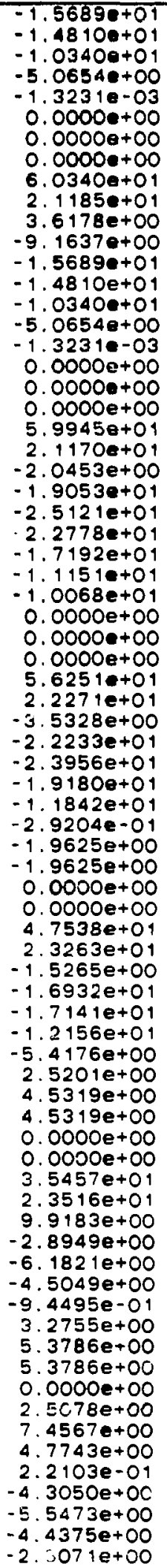 & $\begin{array}{l}8.5097 e+04 \\
1.2255 e+05 \\
1.4463 e+05 \\
1.5498 e+05 \\
1.5748 e+05 \\
1.5978 e+05 \\
1.0874 e+05 \\
1.0874 e+05 \\
6.3366 e+04 \\
1.7991 e+04 \\
8.1127 e+03 \\
3.7699 e+04 \\
8.4955 e+04 \\
1.2273 e+05 \\
1.4482 e+05 \\
1.5514 e+05 \\
1.5746 e+05 \\
1.5978 e+05 \\
9.9773 e+04 \\
9.9773 e+04 \\
5.7507 e+04 \\
1.5242 e+04 \\
6.1370 e+03 \\
3.6649 e+04 \\
8.4813 e+04 \\
1.2290 e+05 \\
1.4502 e+05 \\
1.5530 e+05 \\
1.5743 e+05 \\
1.5956 e+05 \\
8.8824 e+04 \\
8.8824 e+04 \\
5.0377 e+04 \\
1.1930 e+04 \\
3.3543 e+03 \\
3.4823 e+04 \\
8.4317 e+04 \\
1.2283 e+05 \\
1.4502 e+05 \\
1.5550 e+05 \\
1.5715 e+05 \\
1.5881 e+05 \\
8.2692 e+04 \\
8.2692 e+04 \\
4.6131 e+04 \\
9.5703 e+03 \\
7.0230 e+02 \\
3.2915 e+04 \\
8.3537 e+04 \\
1.2233 e+05 \\
1.4451 e+05 \\
1.5495 e+05 \\
1.5643 e+05 \\
1.5790 e+05 \\
8.5353 e+04 \\
8.5353 e+04 \\
4.7597 e+04 \\
9.8416 e+03 \\
1.2552 e+03 \\
3.4109 e+04 \\
8.3641 e+04 \\
1.2077 e+05 \\
1.4227 e+05 \\
1.5262 e+05 \\
1.5448 e+05 \\
1.5634 e+05 \\
6.1089 e+04 \\
5.3093 e+04 \\
1.5097 e+04 \\
1.4688 e+04 \\
1.1374 e+04 \\
4.1356 e+04 \\
8.3892 e+04 \\
1.1719 e+05 \\
1.3770 e+05\end{array}$ & 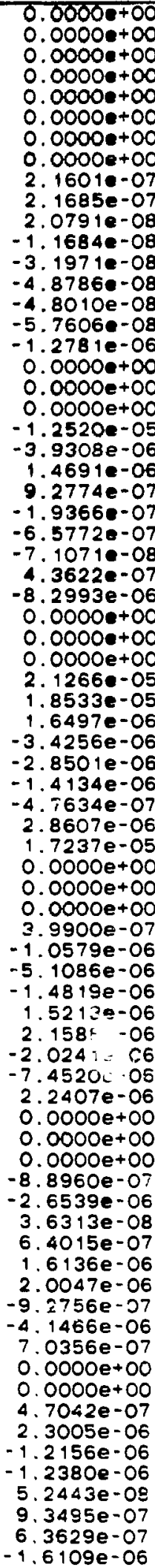 \\
\hline
\end{tabular}


Flow over step * Inflow velocity 1000 " CRAY XMP24 10/30/87 09:0s:41 itere 1 timen 4.00119e-01 cycle= 2216

$M \quad K \quad J \quad I$

$\checkmark v$

P div

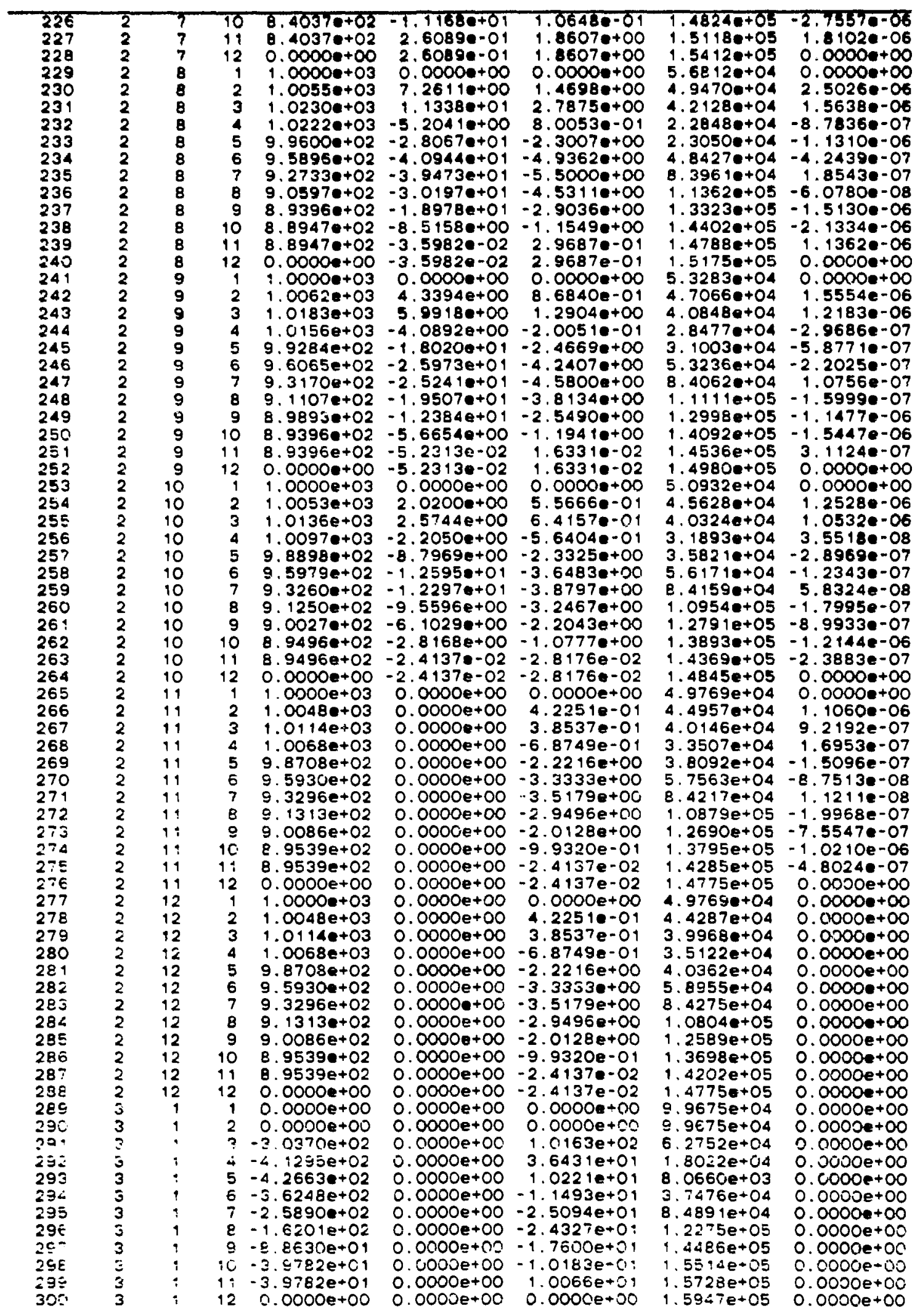


M K U :

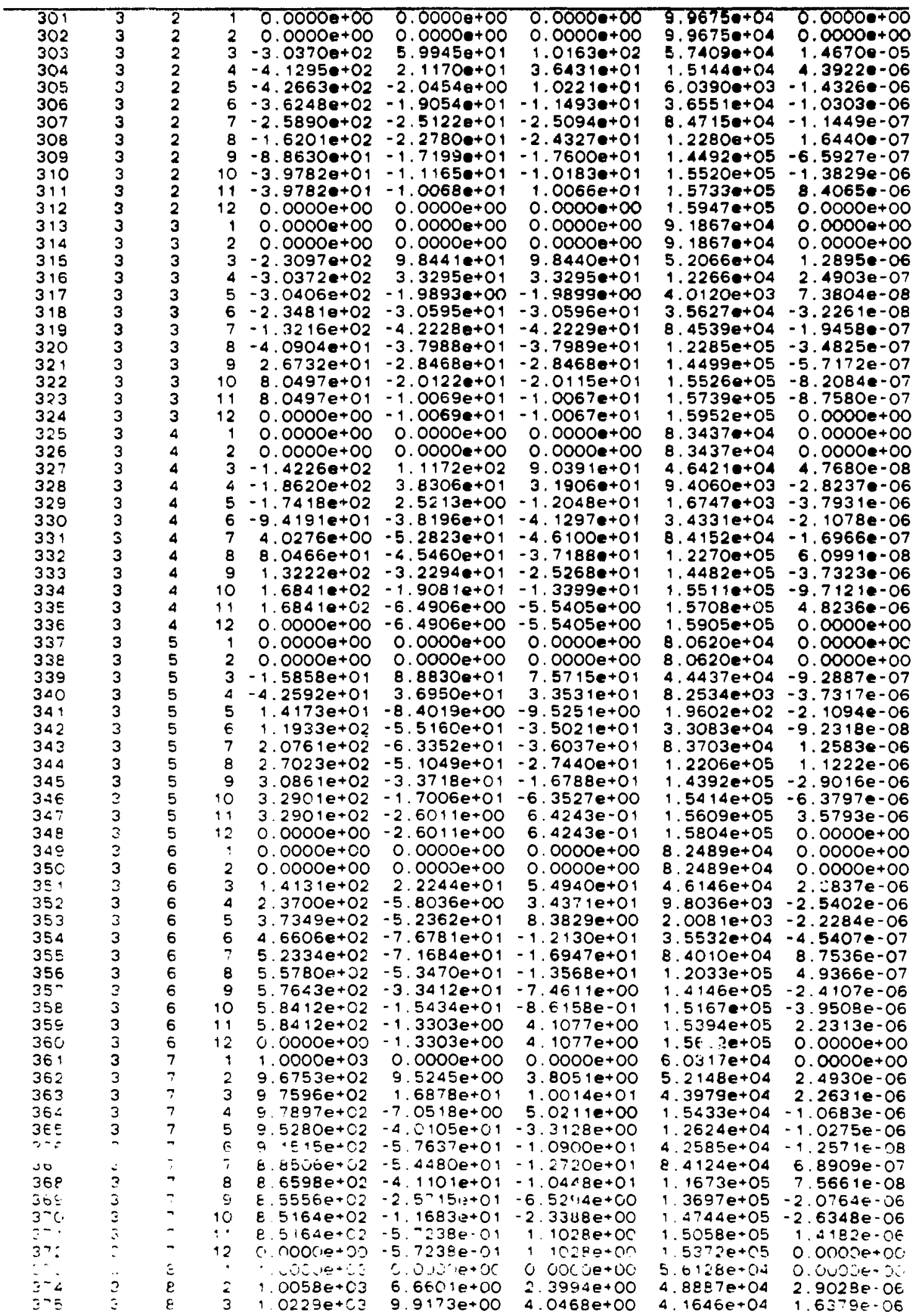


Flow over step "Inflow Veloc1ty = 1000. CRAY XMP24 10/30/87 09:05:41 1tere 1 time: 4.001180-01

M N J I u $\checkmark$ w

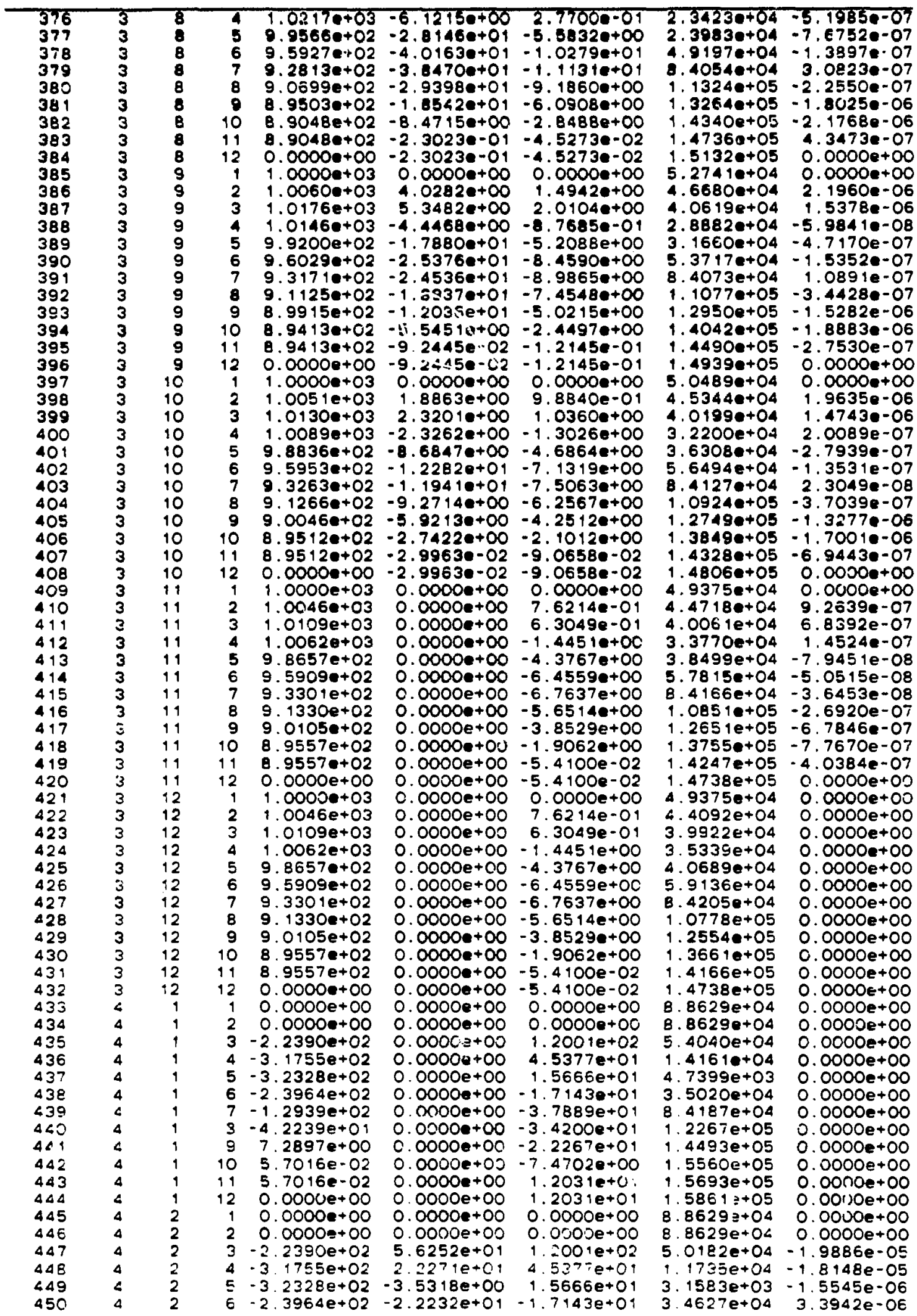


Flow over a step - Inflow velocity - 1000 " CRAY XMP24 10/30/87 09:05:41 iter= $\quad$ time $=4.00119 e-01$

$M \quad K \quad J \quad J$ $\checkmark$

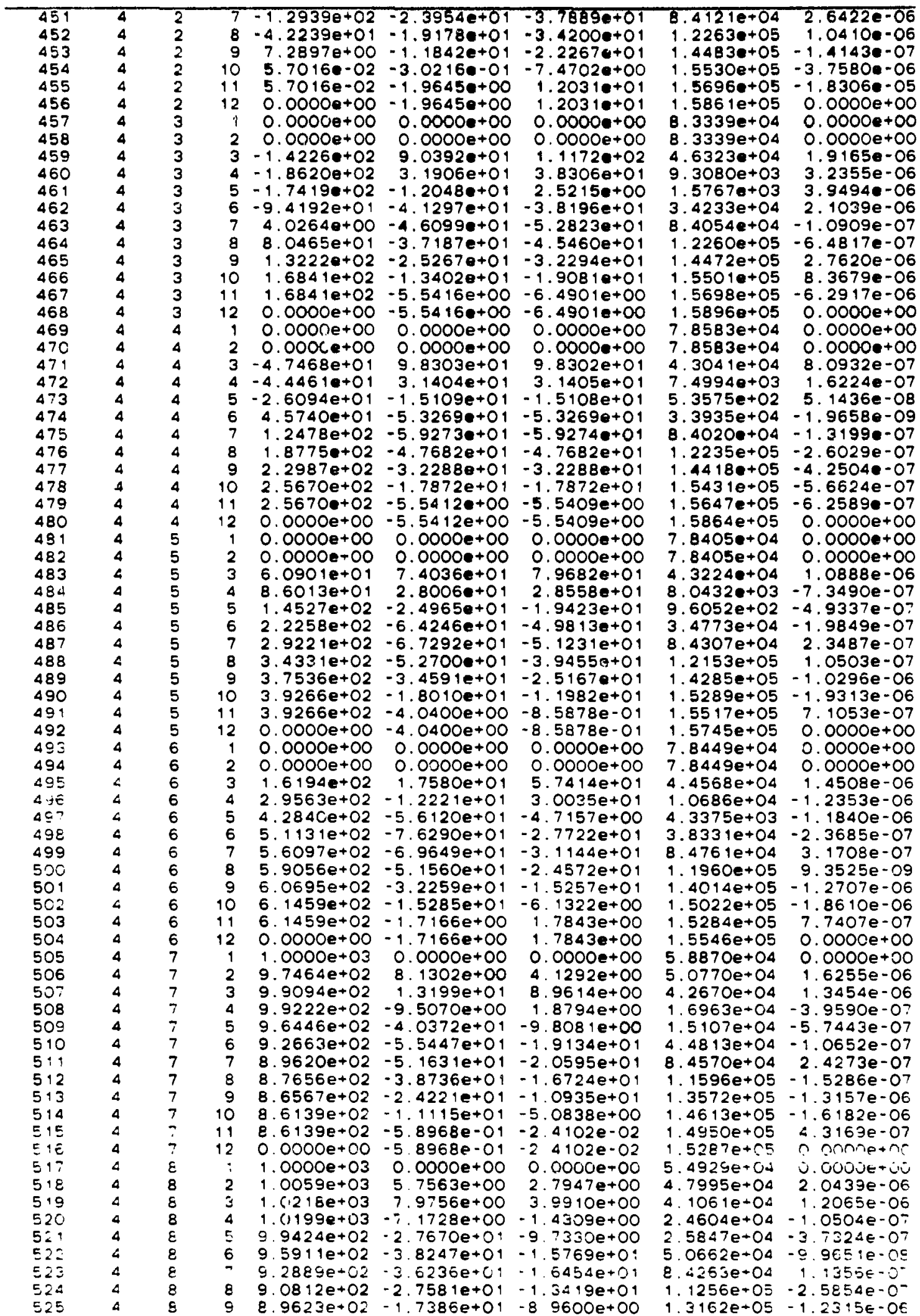




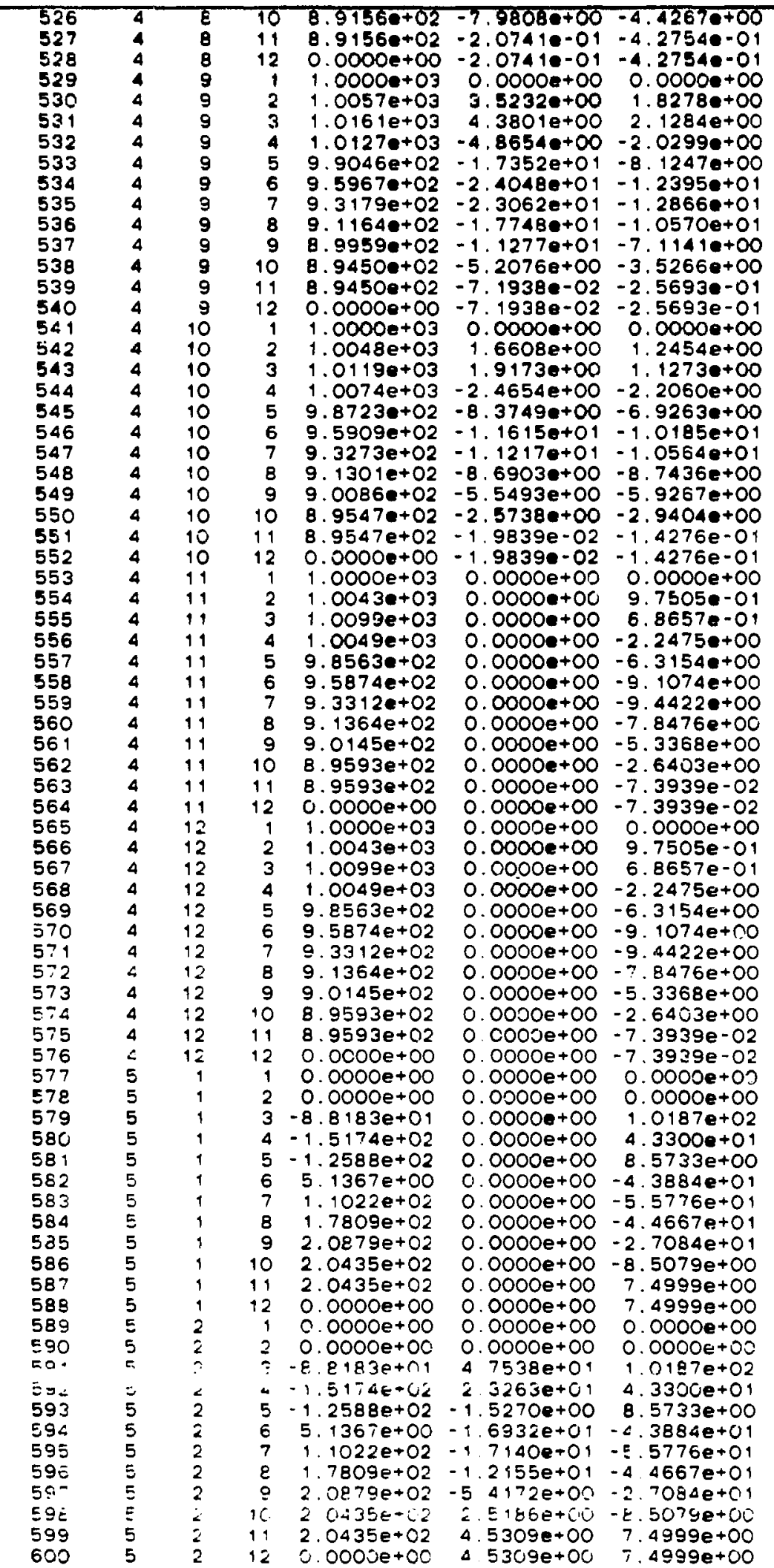

div
$1.42336+05-1.47396-06$ $1.4643 e+05-3.3564 e-08$

$1.5052 e+05 \quad 0.0000++00$

5. $18120+04 \quad 0.0000++00$

$4.6086 e+04 \quad 1.6626 e-06$

$4.03590+04 \quad 1.2031 e-06$

$2.9758 e+04 \quad 1.2685 e-07$

$3.3006 e+04-2.36810-07$

$5.4686 e+04-1.0306-07$

$8.4152 \theta+042.5782 e-08$

$1.1021 e+05-3.0631 e-07$

$1.2867 e+05-1,1086 e-06$

$1.3955 e+05-1.3714 e-06$

$1.4411 e+05-4.2425 e-07$

$1.4867 e+05 \quad 0.0000++00$

$4.9746 e+04 \quad 0.0000 e+00$

$4.4917 e+04 \quad 1.5408 e-06$

$4.0088+04 \quad 1.1866 e-06$

3. $2888 \mathrm{e}+04 \quad 2.6516 \mathrm{e}-07$

$3.7329 e+04-1.3867 e-07$

$5.7180 \mathrm{e}+04-9.3744 \mathrm{e}-08$

B. $4: 37 e+04-1.8986 e-08$

$1.0875 e+05-3.2141 e-07$

$1.2678 e+05-1.0103 e-06$

$1.3775 e+05-1.2904 e-06$

$1.4258 \mathrm{e}+05-6.6821 e \cdot 07$

$1.4741 e+05 \quad 0.0000 e+00$

$4.8724 e+04 \quad 0.0000 e+00$

$4.4365 e+04 \quad 7.7367$ e- 07

$4.0007 e+04 \quad 5.8715 e-07$

$3.4368 e+04 \quad 1.7637 e-07$

$3.9365 e+04-2.2801 e-08$

$5.8374 e+04-3.4755 e-08$

$8.4147 e+04-4.8533 e-08$

$1.0806 e+05-2.3372 e-07$

$1.2587 e+05-5.3948 e-07$

1. $3687 e+05-6.2211 \mathrm{e}-07$

$1.4182 e+05-4.0154 e-07$

$1.4676 \mathrm{e}+05 \quad 0.0000 \mathrm{e}+00$

$4.8724 \mathrm{e}+04 \quad 0.0000 \mathrm{e}+00$

$4.3814 \mathrm{e}+04 \quad 0.0000 \mathrm{e}+00$

$3.9926 \mathrm{e}+04 \quad 0.0000 \mathrm{e}+00$

$3.5847 e+04 \quad 0.0000 e+00$

$41401 \mathrm{e}+04 \quad 0.0000 \mathrm{e}+00$

$5.9568 \mathrm{e}+04 \quad 0.0000 \mathrm{e}+00$

8. $4156 e+04 \quad 0.0000 e+00$

$1.0736 \mathrm{e}+05 \quad 0.0000 \mathrm{e}+00$

$1.2495 e+05 \quad 0.0000 e+00$

$1.3598 \mathrm{e}+05 \quad 0.0000 \mathrm{e}+00$

$1.4105 \mathrm{e}+05 \quad 0.0000 \mathrm{e}+00$

$1.4676 e+05 \quad 0.0000 e+02$

8. $2398 \mathrm{e}+04 \quad 0.0000 \mathrm{e}+00$

8. $2398 \mathrm{e}+04 \quad 0.0000 \mathrm{e}+00$

$4.7433 \mathrm{e}+04 \quad 0.0000 \mathrm{e}+00$

$1.0495 e+04 \quad 0.0000 e+00$

8. $1655 \mathrm{e}+02 \quad 0.0000 \mathrm{e}+00$

$3.2355 \mathrm{e}+04 \quad 0.0000 \mathrm{e}+00$

8. $2980 \mathrm{e}+04 \quad 0.0000 \mathrm{e}+00$

$1.2221 e+05 \quad 0.0000 e+00$

$1.4470 \mathrm{e}+05 \quad 0.0000 \mathrm{e}+00$

$1.5537 e+05 \quad 0.0000 e+00$

$1.5637 \mathrm{e}+05 \quad 0.0000 \mathrm{e}+00$

$1.5761 \mathrm{e}+05 \quad 0.0000 \mathrm{e}+00$

8. $2398 e+04 \quad 0.0000 e+\infty 0$

$8.2398 \mathrm{e}+04 \quad 0.0000 \mathrm{e}+00$

4. $5837 \mathrm{e}+04 \quad 6.6954 \mathrm{e}-07$

$9.2763 \mathrm{e}+03 \quad 1.4357 \mathrm{e}-06$

4. $0828 \mathrm{e}+02 \quad 5.2181 e-06$

3. $2621 \mathrm{e}+04 \quad 1.4389 \mathrm{e}-06$

B. $3243 e+04-1.6771 e-06$

1. $2204 e+05-2.5053 e-06$

1. $4421 e+05 \quad 1.4471 e-05$

$1.54060+05$ E. $536-e-06$

$1.5613 \mathrm{e}+05-3.2 .05 \mathrm{e}-06$

$1.5761 e+050.0000 e+00$ 
Flow over step "Inflow velocity = 1000. CRAY XMP24 10/30/87 09:05:41 iter= 1 time-4.00119e-01

$M \quad K \quad J \quad I \quad U$ $\checkmark$ $w$

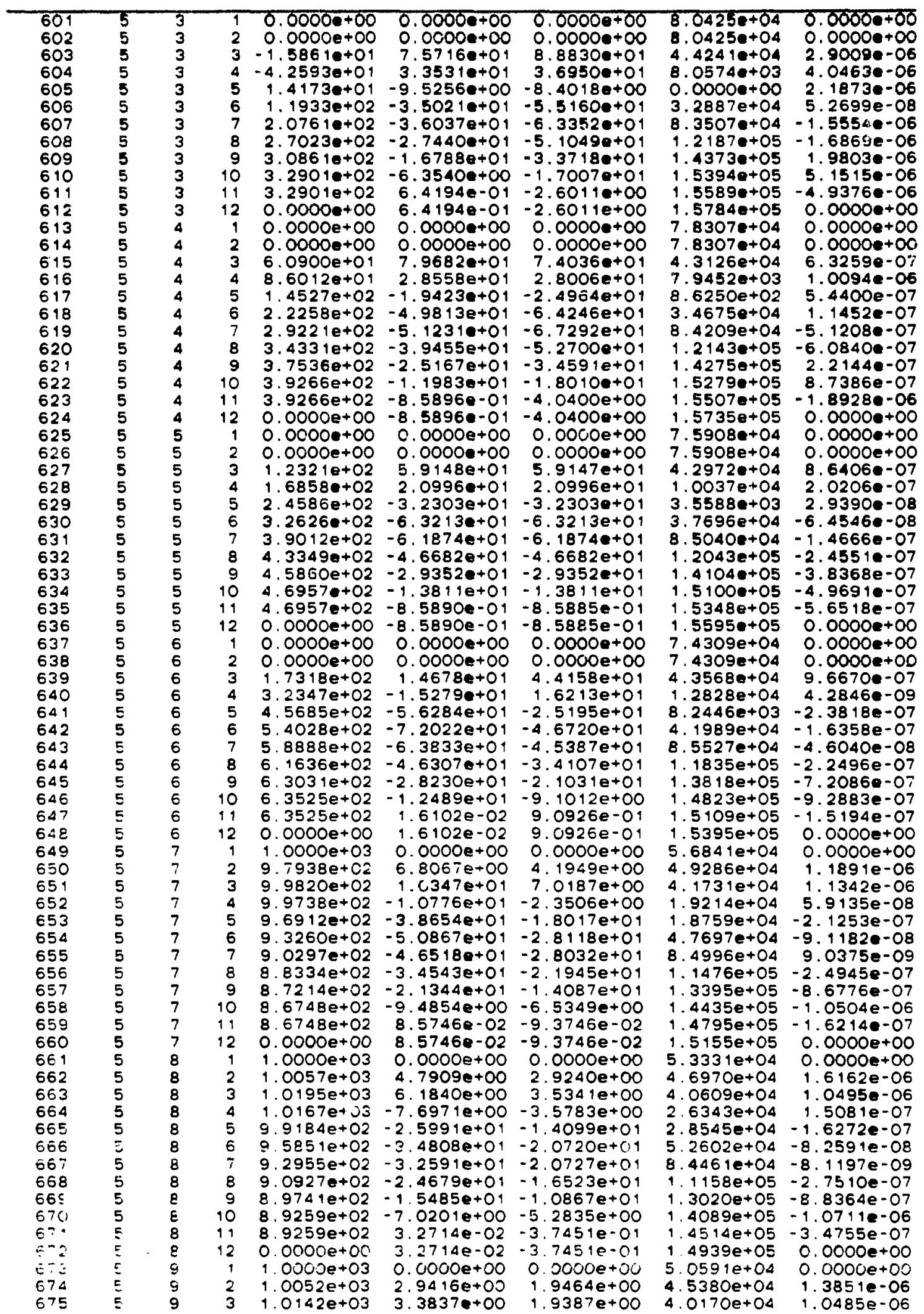


Flow over atep * Inflow veloctty = 1000 * CRAY XMP2A 10/30/87 09:05:11 1tere 1 time=4.001190.01

cycle:

2216

M K $\downarrow$ I $u$ $\checkmark$ $w$

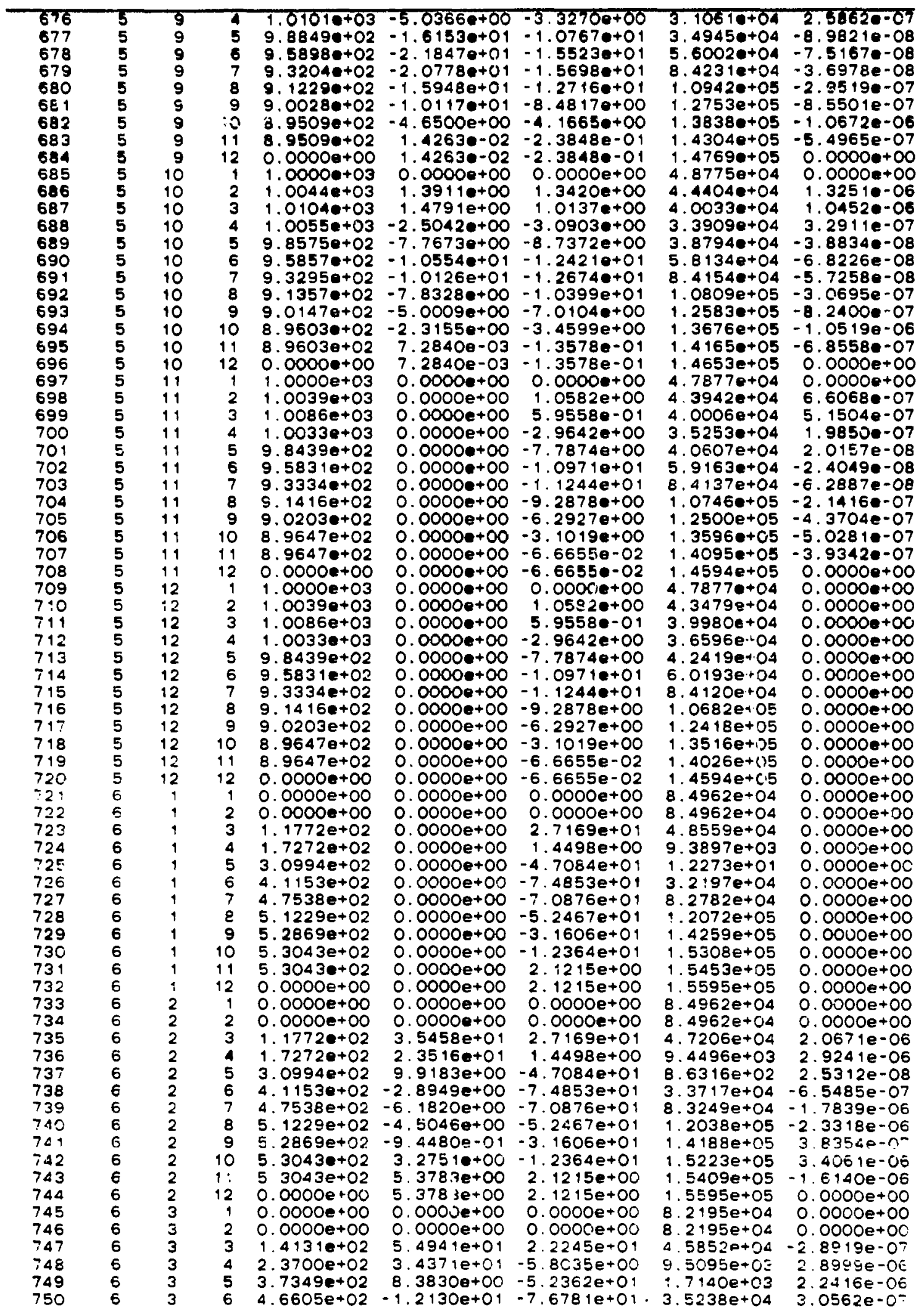


Flow over step Inflow velocity - 1000 - CRAY XMP24 10/30/87 09:05:41 iterx 1 time: 4.00119e-01

$M \quad K \quad J$

$u$

$\checkmark$

$w$

div

\begin{tabular}{|c|c|c|c|c|c|c|c|c|}
\hline $\begin{array}{l}751 \\
752 \\
753 \\
754 \\
755 \\
756 \\
757 \\
758 \\
759 \\
760 \\
761 \\
762 \\
763 \\
764 \\
765 \\
766 \\
767 \\
768 \\
769 \\
770 \\
771 \\
772 \\
773 \\
774 \\
775 \\
776 \\
777 \\
778 \\
779 \\
780 \\
781 \\
782 \\
783 \\
784 \\
785 \\
786 \\
787 \\
788 \\
789 \\
790 \\
791 \\
792 \\
793 \\
794 \\
795 \\
796 \\
797 \\
799 \\
799 \\
800 \\
801 \\
802 \\
803 \\
804 \\
805 \\
806 \\
807 \\
808 \\
809 \\
810 \\
811 \\
812 \\
813 \\
814 \\
815 \\
816 \\
817 \\
818 \\
810 \\
820 \\
829 \\
822 \\
672 \\
824 \\
E 25\end{array}$ & 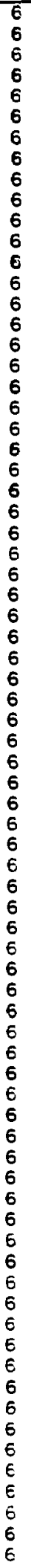 & $\begin{array}{l}3 \\
3 \\
3 \\
3\end{array}$ & 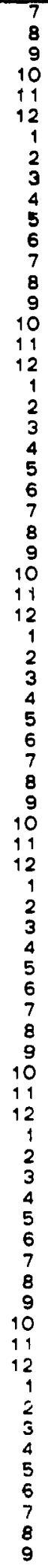 & $\begin{array}{l}5.2334 e+02 \\
5.5780 e+02 \\
5.7643 e+02 \\
5.8412 e+02 \\
5.8412 e+02 \\
0.0000 e+00 \\
0.0000++00 \\
0.0000 e+00 \\
1.6194 e+02 \\
2.9563 e+02 \\
4.2840 e+02 \\
5.1131 e+02 \\
5.6097 e+02 \\
5.9056 e+02 \\
6.0696 e+02 \\
6.1459 e+02 \\
6.1459 e+02 \\
0.0000 e+00 \\
0.0000 e+00 \\
0.0000 e+00 \\
1.7318 e+02 \\
3.2347 e+02 \\
4.5685 e+02 \\
5.4028 e+02 \\
5.8888 e+02 \\
6.1636 e+02 \\
6.3031 e+02 \\
6.3525 e+02 \\
6.3525 e+02 \\
0.0000 e+00 \\
0.0000 e+00 \\
0.0000 e+00 \\
1.8449 e+02 \\
3.8676 e+02 \\
5.4729 e+02 \\
6.3519 e+02 \\
6.7904 e+02 \\
7.0035 e+02 \\
7.0966 e+02 \\
7.1197 e+02 \\
7.1197 e+02 \\
0.0000 e+00 \\
1.0000 e+03 \\
9.8194 e+02 \\
9.9756 e+02 \\
9.9560 e+02 \\
9.7013 e+02 \\
9.3762 e+02 \\
9.1033 e+02 \\
8.9152 e+02 \\
8.8045 e+02 \\
8.7571 e+02 \\
8.7571 e+02 \\
0.0000 e+00 \\
1.0000 e+03 \\
1.0051 e+03 \\
1.0164 e+03 \\
1.0125 e+03 \\
9.8902 e+02 \\
9.5797 e+02 \\
9.3048 e+02 \\
9.1073 e+02 \\
8.98389 e+02 \\
1.0000 e+02 \\
1.0045 e+03 \\
1.0118 e+03 \\
1.0070 e+03 \\
9.8632 e+02 \\
9.5837 e+02 \\
9.3250 e+02 \\
9.1318 e+02 \\
9.0121 e+02\end{array}$ & 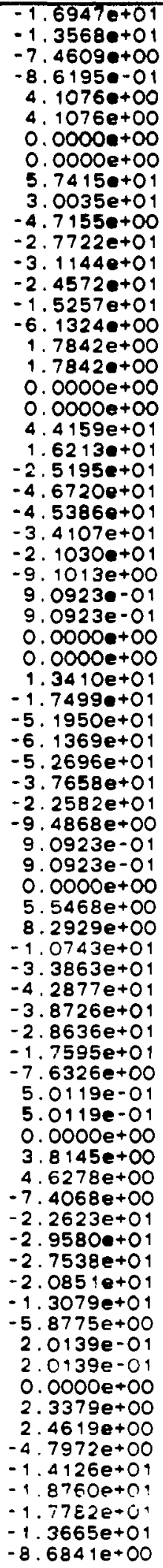 & 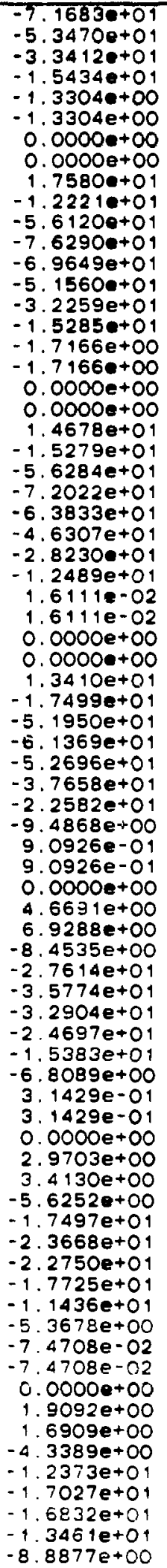 & $\begin{array}{l}8.3716 e+04 \\
1.2003 e+05 \\
1.4117 e+05 \\
1.5138 e+05 \\
1.5365 e+05 \\
1.5592 e+05 \\
7.8253 e+04 \\
7.8253 e+04 \\
4.4372 e+04 \\
1.0490 e+04 \\
4.1415 e+03 \\
3.8135 e+04 \\
8.4565 e+04 \\
1.1940 e+05 \\
1.3994 e+05 \\
1.5003 e+05 \\
1.5264 e+05 \\
1.5526 e+05 \\
7.4211 e+04 \\
7.4211 e+04 \\
4.3470 e+04 \\
1.2730 e+04 \\
8.1465 e+03 \\
4.1891 e+04 \\
8.5429 e+04 \\
1.1825 e+05 \\
1.3808 e+05 \\
1.4813 e+05 \\
1.5099 e+05 \\
1.5385 e+05 \\
7.0928 e+04 \\
7.0928 e+04 \\
4.3161 e+04 \\
1.5394 e+04 \\
1.3680 e+04 \\
4.6262 e+04 \\
8.5810 e+04 \\
1.1621 e+05 \\
1.3534 e+05 \\
1.4548 e+05 \\
1.4874 e+05 \\
1.5199 e+05 \\
5.4364 e+04 \\
4.7782 e+04 \\
4.1201 e+04 \\
2.2221 e+04 \\
2.3793 e+04 \\
5.0998 e+04 \\
8.5093 e+04 \\
1.1298 e+05 \\
1.3159 e+05 \\
1.4206 e+05 \\
1.4598 e+05 \\
1.4991 e+05 \\
5.1467 e+04 \\
4.5901 e+04 \\
4.0335 e+04 \\
2.8651 e+04 \\
3.2017 e+04 \\
5.4800 e+04 \\
8.4507 e+04 \\
1.1028 e+05 \\
1.2843 e+05 \\
1.3913 e+05 \\
1.4358 e+05 \\
1.05050+05 \\
4.9188 e+04 \\
4.4635 e+04 \\
4.0082 e+04 \\
3.2735 e+04 \\
3.7345 e+04 \\
5.7504 e+04 \\
8.4245 e+04 \\
1.0844 e+05 \\
1.2617 e+05\end{array}$ & 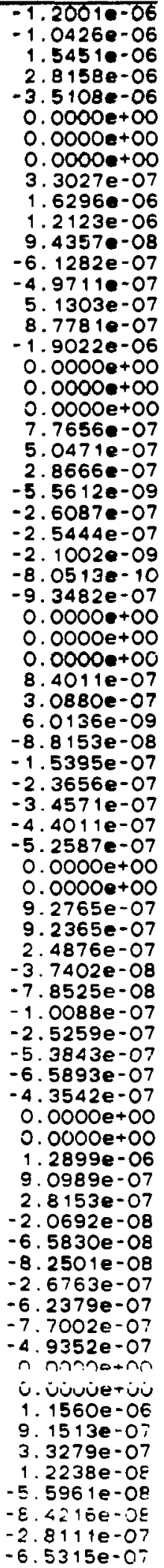 \\
\hline
\end{tabular}




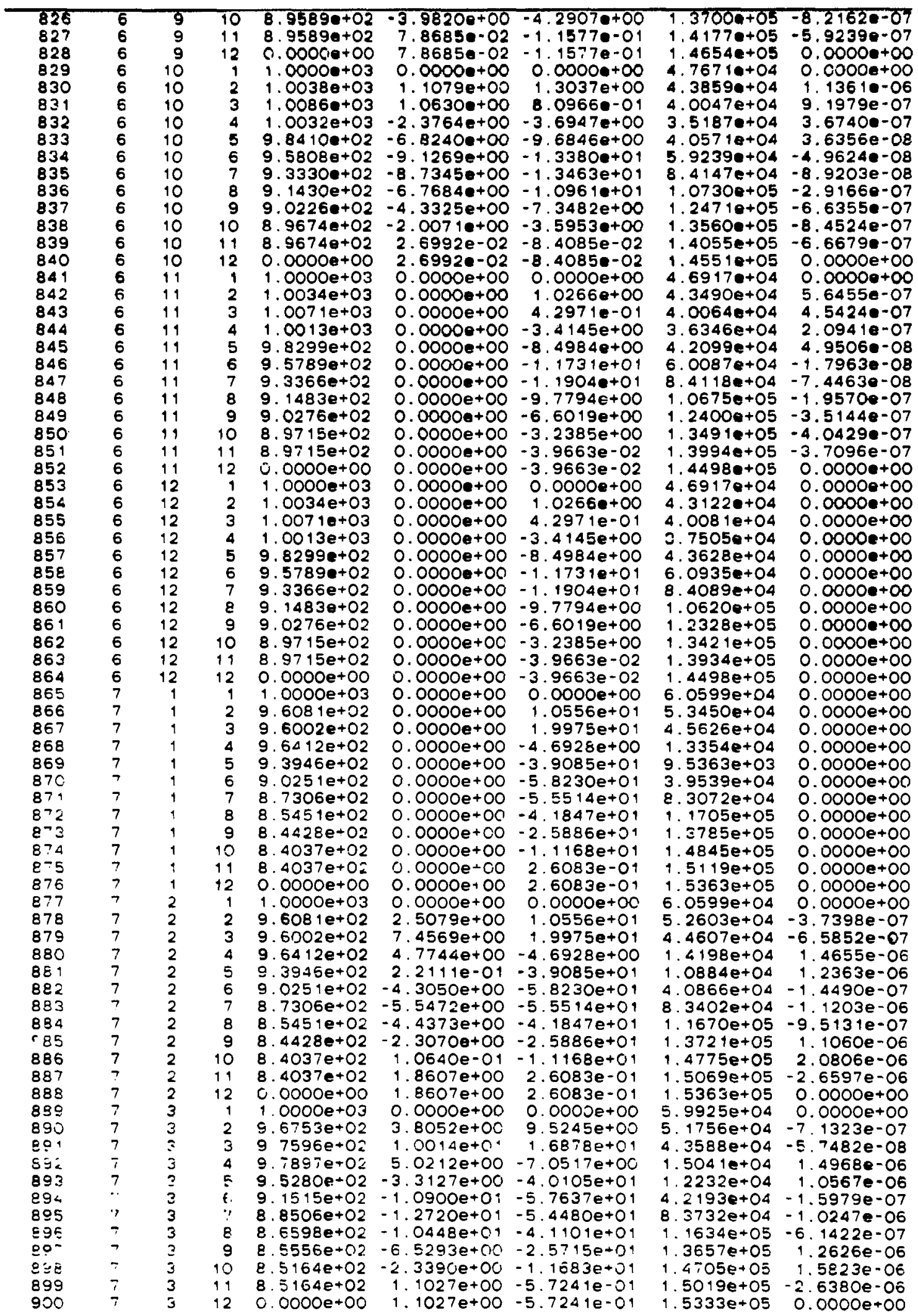


Flow over a step "Inflow velocity = 1000" CRAY XMP24 10/30/87 09:05:41 iter. , time. 4.001180-01

cycle= 2216

M J 1

$v$

$w$

div

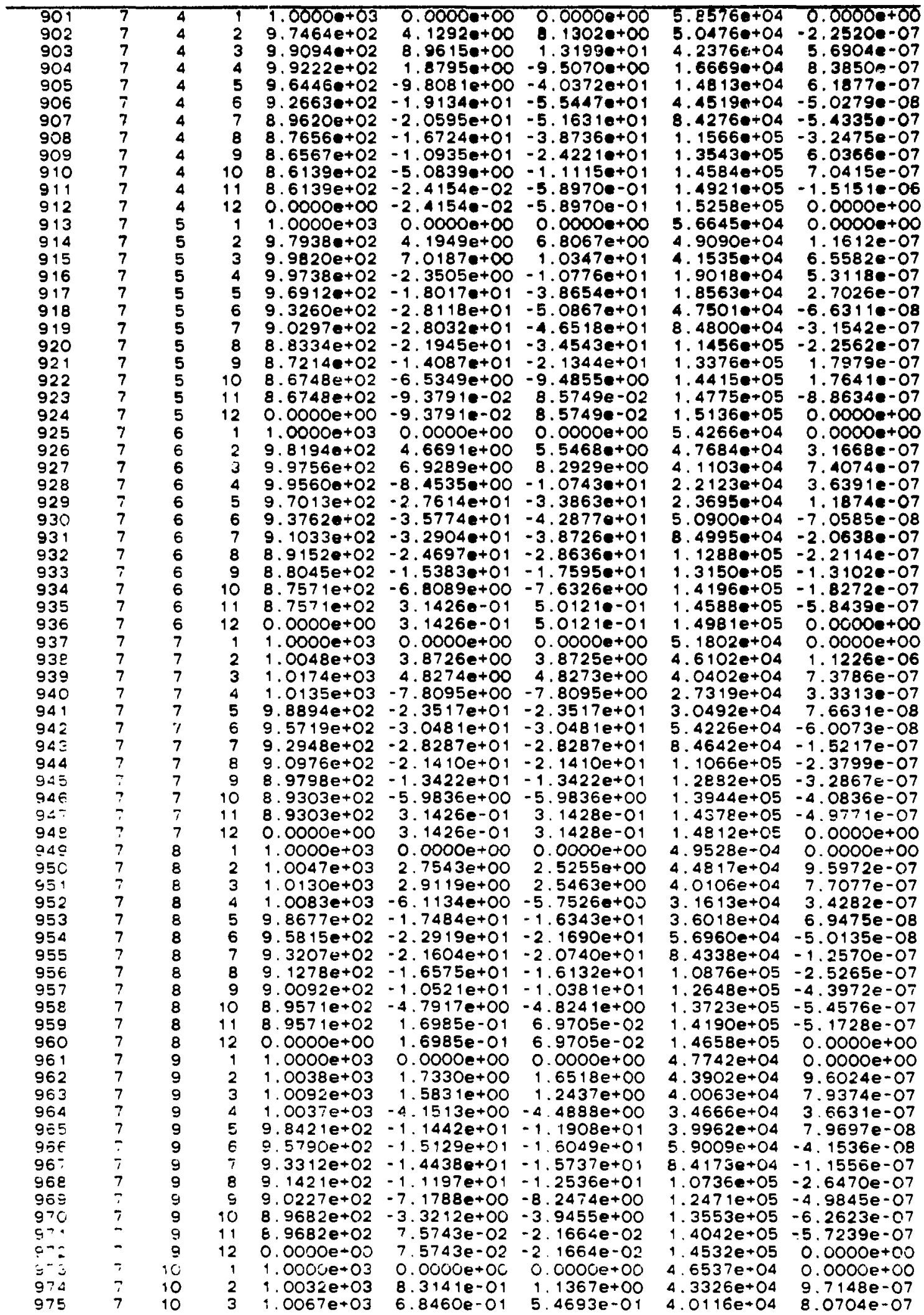


Flow over a top "Inflow velocity - 1000. CRAY XMP24 $10 / 30 / 87$ 09:05:41 iter. 1 timex 4.00119e-01 cycles 2216

M K J I U U v W

oiv

\begin{tabular}{|c|c|c|c|c|c|c|c|c|}
\hline $\begin{array}{l}975 \\
977 \\
978 \\
979 \\
980 \\
981 \\
982 \\
983 \\
984 \\
985 \\
986 \\
987 \\
988 \\
989 \\
990 \\
991 \\
992 \\
993 \\
994 \\
995 \\
996 \\
997 \\
998 \\
999 \\
1000 \\
1001 \\
1002 \\
1003 \\
1004 \\
1005 \\
1006 \\
1007 \\
1008 \\
1009 \\
1010 \\
1011 \\
1012 \\
1013 \\
1014 \\
1015 \\
1016 \\
1017 \\
1018 \\
1019 \\
1020 \\
1021 \\
1022 \\
1023 \\
1024 \\
1025 \\
1026 \\
1027 \\
1028 \\
1029 \\
1030 \\
1031 \\
1032 \\
1033 \\
1034 \\
1035 \\
1036 \\
1037 \\
1038 \\
1039 \\
1040 \\
1041 \\
1042 \\
1043 \\
104 . \\
1045 \\
10245 \\
1007 \\
1045 \\
1045 \\
1050\end{array}$ & $\begin{array}{l}7 \\
7 \\
7 \\
7 \\
7 \\
7 \\
7 \\
7 \\
7 \\
7 \\
7 \\
7 \\
7 \\
7 \\
7 \\
7 \\
7 \\
7 \\
7 \\
7 \\
7 \\
7 \\
7 \\
7 \\
7 \\
7 \\
7 \\
7 \\
7 \\
7 \\
7 \\
7 \\
8 \\
8 \\
8 \\
8 \\
8 \\
8 \\
8 \\
8 \\
8 \\
8 \\
8 \\
8 \\
8 \\
8 \\
8 \\
8 \\
8 \\
8 \\
8\end{array}$ & 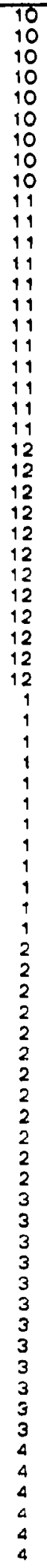 & 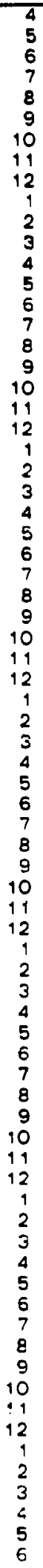 & $\begin{array}{l}1.0008 e+03 \\
9.8246 e+02 \\
9.5766 e+02 \\
9.3376 e+02 \\
9.1513 e+02 \\
9.0314 e+02 \\
8.9754 e+02 \\
8.9754 e+02 \\
0.0000 e+00 \\
1.0000 e+03 \\
1.0029 e+03 \\
1.0055 e+03 \\
9.9930 e+02 \\
9.8158 e+02 \\
9.5751 e+02 \\
9.3406 e+02 \\
9.1558 e+02 \\
9.0358 e+02 \\
8.9791 e+02 \\
8.9791 e+02 \\
0.0000 e+00 \\
1.0000 e+03 \\
1.0029 e+03 \\
1.0055 e+03 \\
9.9930 e+02 \\
9.8158 e+02 \\
9.5751 e+02 \\
9.3406 e+02 \\
9.1558 e+02 \\
9.0358 e+02 \\
8.9791 e+02 \\
9.9791 e+02 \\
0.0000 e+00 \\
1.0000 e+03 \\
1.0055 e+03 \\
1.0230 e+03 \\
1.0222 e+03 \\
9.9600 e+02 \\
9.5896 e+02 \\
9.2733 e+02 \\
9.0597 e+02 \\
8.9396 e+02 \\
8.8947 e+02 \\
8.8947 e+02 \\
0.0000 e+00 \\
1.0000 e+03 \\
1.0055 e+03 \\
1.0230 e+03 \\
1.0222 e+03 \\
9.9600 e+02 \\
9.5836 e+02 \\
9.2733 e+02 \\
9.00497 e+02 \\
8.9396 e+02 \\
8.8947 e+02 \\
8.8947 e+02 \\
0.0000 e+00 \\
1.0000 e+03 \\
1.0058 e+03 \\
1.0229 e+03 \\
1.0217 e+03 \\
9.9566 e+02 \\
9.5927 e+02 \\
9.2813 e+02 \\
9.0099 e+02 \\
1.0218 e+00+02 \\
1.0199 e+03 \\
9.9424 e+02 \\
9.5911 e+02\end{array}$ & 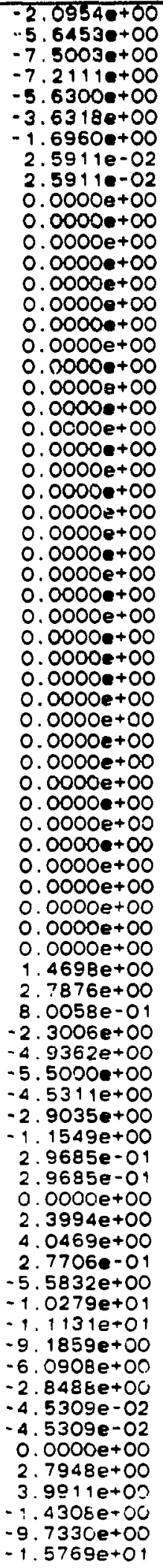 & 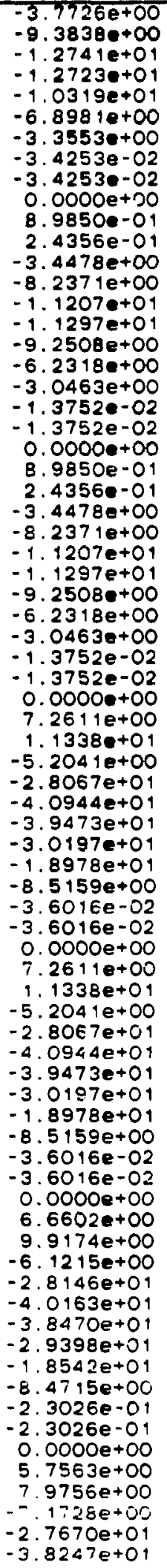 & $\begin{array}{l}3.6599 e+04 \\
4.2469 e+04 \\
6.0366 e+04 \\
8.4104 e+04 \\
1.0645 e+05 \\
1.2353 e+05 \\
1.3438 e+05 \\
1.3940 e+05 \\
1.4443 e+05 \\
4.5934 e+04 \\
4.3050 e+04 \\
4.0166 e+04 \\
3.7534 e+04 \\
4.3684 e+04 \\
6.1040 e+04 \\
8.4082 e+04 \\
1.0600 e+05 \\
1.2294 e+05 \\
1.3380 e+05 \\
1.3889 e+05 \\
1.4398 e+05 \\
4.5934 e+04 \\
4.2773 e+04 \\
4.0216 e+04 \\
3.8469 e+04 \\
4.4898 e+04 \\
6.1715 e+04 \\
8.4060 e+04 \\
1.0556 e+05 \\
1.2235 e+05 \\
1.3322 e+05 \\
1.3837 e+05 \\
1.4398 e+05 \\
5.6224 e+04 \\
4.9367 e+04 \\
4.1924 e+04 \\
2.1587 e+04 \\
2.1430 e+04 \\
4.6971 e+04 \\
8.3182 e+04 \\
1.1333 e+05 \\
1.3313 e+05 \\
1.4395 e+05 \\
1.4773 e+05 \\
1.5117 e+05 \\
5.6224 e+04 \\
4.8822 e+04 \\
4.1540 e+04 \\
2.2260 e+04 \\
2.2461 e+04 \\
4.7939 e+04 \\
8.3373 e+04 \\
1.1304 e+05 \\
1.3264 e+05 \\
1.4343 e+05 \\
1.4730 e+05 \\
1.5117 e+05 \\
5.5638 e+04 \\
4.8397 e+04 \\
4.1156 e+04 \\
2.2933 e+04 \\
2.3493 e+04 \\
4.8707 e+04 \\
8.3564 e+04 \\
1.1275 e+05 \\
1.3215 e+05 \\
1.4251 e+05 \\
1.4687 e+05 \\
1.5083 e+05 \\
5.4537 e+04 \\
4.7603 e+04 \\
4.0669 e+04 \\
2.4212 e+04 \\
2.5455 e+04 \\
5.0270 e+04\end{array}$ & 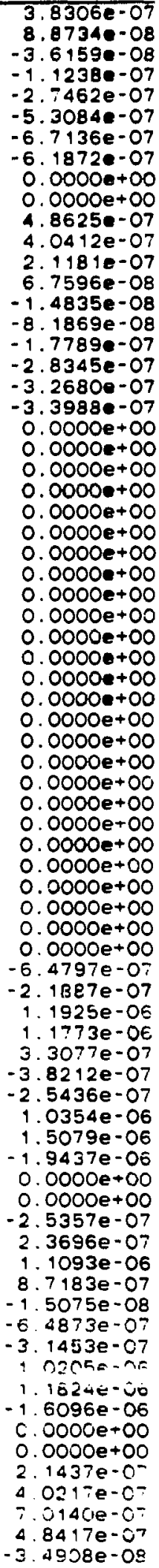 \\
\hline
\end{tabular}


Flow over a stop "Inflow velocity. 1000. CRAY XMP24 10/30/87 09:05:41, iter. 1 time* 4.00119e-01 cyclen 2216 M K J I $u$ $\checkmark$ $w$ div

\begin{tabular}{|c|c|c|c|c|c|c|c|c|}
\hline $\begin{array}{l}1051 \\
1052 \\
1053 \\
1054 \\
1055 \\
1056 \\
1057 \\
1058 \\
1059 \\
1060 \\
1061 \\
1062 \\
1063 \\
1064 \\
1065 \\
1066 \\
1067 \\
1068 \\
1069 \\
1070 \\
1071 \\
1072 \\
1073 \\
1074 \\
1075 \\
1076 \\
1077 \\
1078 \\
1079 \\
1080 \\
1081 \\
1082 \\
1083 \\
1084 \\
1085 \\
1086 \\
1087 \\
1088 \\
1089 \\
1090 \\
1091 \\
1092 \\
1093 \\
1094 \\
1095 \\
1096 \\
1097 \\
1098 \\
1099 \\
1100 \\
1101 \\
1102 \\
1103 \\
1104 \\
1105 \\
1106 \\
11107 \\
1108 \\
1109 \\
1110 \\
1111 \\
1112 \\
1113 \\
1114 \\
1115 \\
1116 \\
1117 \\
1118 \\
1119 \\
1120 \\
1121 \\
1122 \\
1123 \\
1124 \\
1125\end{array}$ & $\begin{array}{l}8 \\
8 \\
8 \\
8 \\
8 \\
8 \\
8 \\
8 \\
8 \\
8 \\
8 \\
8 \\
8 \\
8 \\
8 \\
8 \\
8 \\
8 \\
8 \\
8 \\
8 \\
8 \\
8 \\
8 \\
8 \\
8 \\
8 \\
8 \\
8 \\
8 \\
8 \\
8 \\
8 \\
8 \\
8 \\
8 \\
8 \\
8 \\
8 \\
8 \\
8 \\
8 \\
8 \\
8 \\
8 \\
8 \\
8 \\
8 \\
8 \\
8 \\
8\end{array}$ & 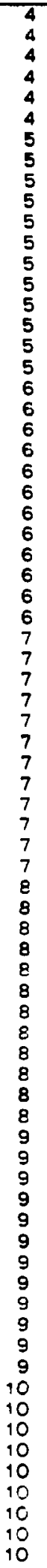 & $\begin{array}{r}9 \\
8 \\
9 \\
10 \\
11 \\
12\end{array}$ & $\begin{array}{l}9.2889 e+02 \\
9.0812 e+02 \\
8.9623 e+02 \\
8.9156 e+02 \\
8.9156 e+02 \\
0.0000++00 \\
1.0000 e+03 \\
1.0057 e+03 \\
1.0195 e+03 \\
1.0167 e+03 \\
9.9184 e+02 \\
9.5851 e+02 \\
9.2955 e+02 \\
9.0927 e+02 \\
8.9741+02 \\
8.9259 e+02 \\
8.9259 e+02 \\
0.0000 e+00 \\
1.0000 e+03 \\
1.0051 e+03 \\
1.0164 e+03 \\
1.0125 e+03 \\
9.8902 e+02 \\
9.5797 e+02 \\
9.3048 e+02 \\
9.1073 e+02 \\
8.9889 e+02 \\
8.9388 e+02 \\
8.9388 e+02 \\
0.0000 e+00 \\
1.0000 e+03 \\
1.0047 e+03 \\
1.0130 e+03 \\
1.0083 e+03 \\
9.8677 e+02 \\
9.5815 e+02 \\
9.3207 e+02 \\
9.1278 e+02 \\
9.0092 e+02 \\
8.9571 e+02 \\
8.9571 e+02 \\
0.0000 e+00 \\
1.0000 e+03 \\
1.0039 e+03 \\
1.0095 e+03 \\
1.0041 e+03 \\
9.8429 e+02 \\
9.5783 e+02 \\
9.3302 e+02 \\
9.1415 e+02 \\
9.0224 e+02 \\
8.9682 e+02 \\
8.9682 e+02 \\
0.0000 e+00 \\
1.0000 e+03 \\
1.0031 e+03 \\
1.0067 e+03 \\
1.0007 e+03 \\
9.8233 e+02 \\
9.5754 e+02 \\
9.3374 e+02 \\
9.1521 e+02 \\
9.0328 e+02 \\
8.9771 e+02 \\
8.9771 e+02 \\
0.0000 e+00 \\
1.0000 e+03 \\
1.0026 e+03 \\
1.0049 e+03 \\
9.9852 e+02 \\
9.8099 e+02 \\
9.5722 e+02 \\
9.3423 e+02 \\
9.1595 e+02 \\
9.0401 e+02\end{array}$ & $\begin{array}{r}-1.6454 e+01 \\
-1.3419 e+01 \\
-8.9600++00 \\
-4.4268 e+00 \\
-4.2758 e-01 \\
-4.2758 e-01 \\
0.0000 e+00 \\
2.9241 e+00 \\
3.5341 e+00 \\
-3.5783 e+00 \\
-1.4099 e+01 \\
-2.0720 e+01 \\
-2.0727 e+01 \\
-1.6523 e+01 \\
-1.0867 e+01 \\
-5.2835 e+00 \\
-3.7454 e-01 \\
-3.7454 e-01 \\
0.0000 e+00 \\
2.9703 e+00 \\
3.4130 e+00 \\
-5.6252 e+00 \\
-1.7497 e+01 \\
-2.3668 e+01 \\
-2.2750 e+01 \\
-1.7725 e+01 \\
-1.1436 e+01 \\
-5.3678 e+00 \\
-7.4737 e-02 \\
-7.4737 e-02 \\
0.0000 e+00 \\
2.5255 e+00 \\
2.5463 e+00 \\
-5.7525 e+00 \\
-1.6343 e+01 \\
-2.1690 e+01 \\
-2.0740 e+01 \\
-1.6132 e+01 \\
-1.0381 e+01 \\
-4.8241 e+00 \\
6.9685 e-02 \\
6.9685 e-02 \\
0.0000 e+00 \\
1.8809 e+00 \\
1.6108 e+00 \\
-4.8485 e+00 \\
-1.3049 e+01 \\
-1.7279 e+01 \\
-1.6605 e+01 \\
-1.2986 e+01 \\
-8.3962 e+00 \\
-3.9219 e+00 \\
6.9689 e-02 \\
6.9689 e-02 \\
0.0000 e+00 \\
1.2183 e+00 \\
8.8754 e-01 \\
-3.4344 e+00 \\
-8.9216 e+00 \\
-1.1832 e+01 \\
-1.1442 e+01 \\
-9.0028 e+00 \\
-5.8535 e+00 \\
-2.7553 e+00 \\
3.8712 e-02 \\
3.8712 e-02 \\
0.0000 e+00 \\
5.9352 e-01 \\
3.8208 e-01 \\
-1.7703 e+00 \\
-4.5046 e+00 \\
-5.9833 e+00 \\
-5.8119 e+00 \\
-4.5930 e+00 \\
-2.9987 e+00\end{array}$ & 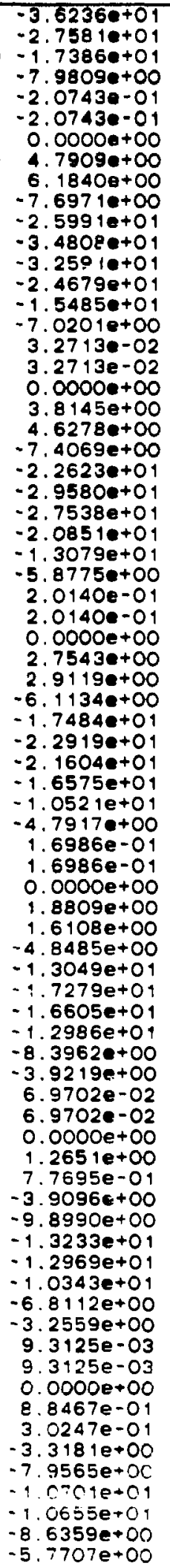 & $\begin{array}{l}8.3871 e+04 \\
1.1217 e+05 \\
1.3123 e+05 \\
1.4194 e+05 \\
1.4604 e+05 \\
1.5013 e+05 \\
5.3037 e+04 \\
4.6676 e+04 \\
4.0315 e+04 \\
2.6049 e+04 \\
2.8251 e+04 \\
5.2308 e+04 \\
8.4167 e+04 \\
1.1129 e+05 \\
1.2991 e+05 \\
1.4060 e+05 \\
1.4485 e+05 \\
1.4910 e+05 \\
5.1271 e+04 \\
4.5705 e+04 \\
4.0139 e+04 \\
2.8455 e+04 \\
3.1821 e+04 \\
5.4604 e+04 \\
8.4311 e+04 \\
1.1009 e+05 \\
1.2824 e+05 \\
1.3894 e+05 \\
1.4339 e+05 \\
1.4783 e+05 \\
4.9430 e+04 \\
4.4719 e+04 \\
4.0008 e+04 \\
3.1515 e+04 \\
3.5920 e+04 \\
5.6862 e+04 \\
8.4240 e+04 \\
1.0866 e+05 \\
1.2638 e+05 \\
1.3713 e+05 \\
1.4181 e+05 \\
1.4648 e+05 \\
4.7750 e+04 \\
4.3880 e+04 \\
4.0010 e+04 \\
3.4319 e+04 \\
3.9567 e+04 \\
5.8820 e+04 \\
8.4135 e+04 \\
1.0736 e+05 \\
1.2469 e+05 \\
1.3548 e+05 \\
1.4034 e+05 \\
1.4521 e+05 \\
4.6406 e+04 \\
4.3255 e+04 \\
4.0104 e+04 \\
3.6494 e+04 \\
4.2376 e+04 \\
6.0351 e+04 \\
8.4069 e+04 \\
1.0633 e+05 \\
1.2333 e+05 \\
1.3414 e+05 \\
1.3915 e+05 \\
1.4416 e+05 \\
4.5483 e+04 \\
4.2850 e+04 \\
4.0216 e+04 \\
3.7947 e+04 \\
4.4246 e+04 \\
6.1394 e+04 \\
E .-05=+04 \\
1.0563 e+05 \\
1.2240 e+05\end{array}$ & 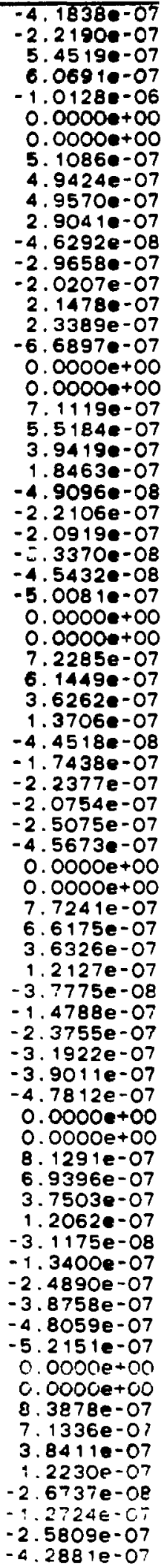 \\
\hline
\end{tabular}


Flow over a step - Inflow velocity " 1000. CRAY XMP24 10/30/87 09:05:41 1ter: 4 timed 4.00119e-01
M K J I
$u$
$v$
aiv

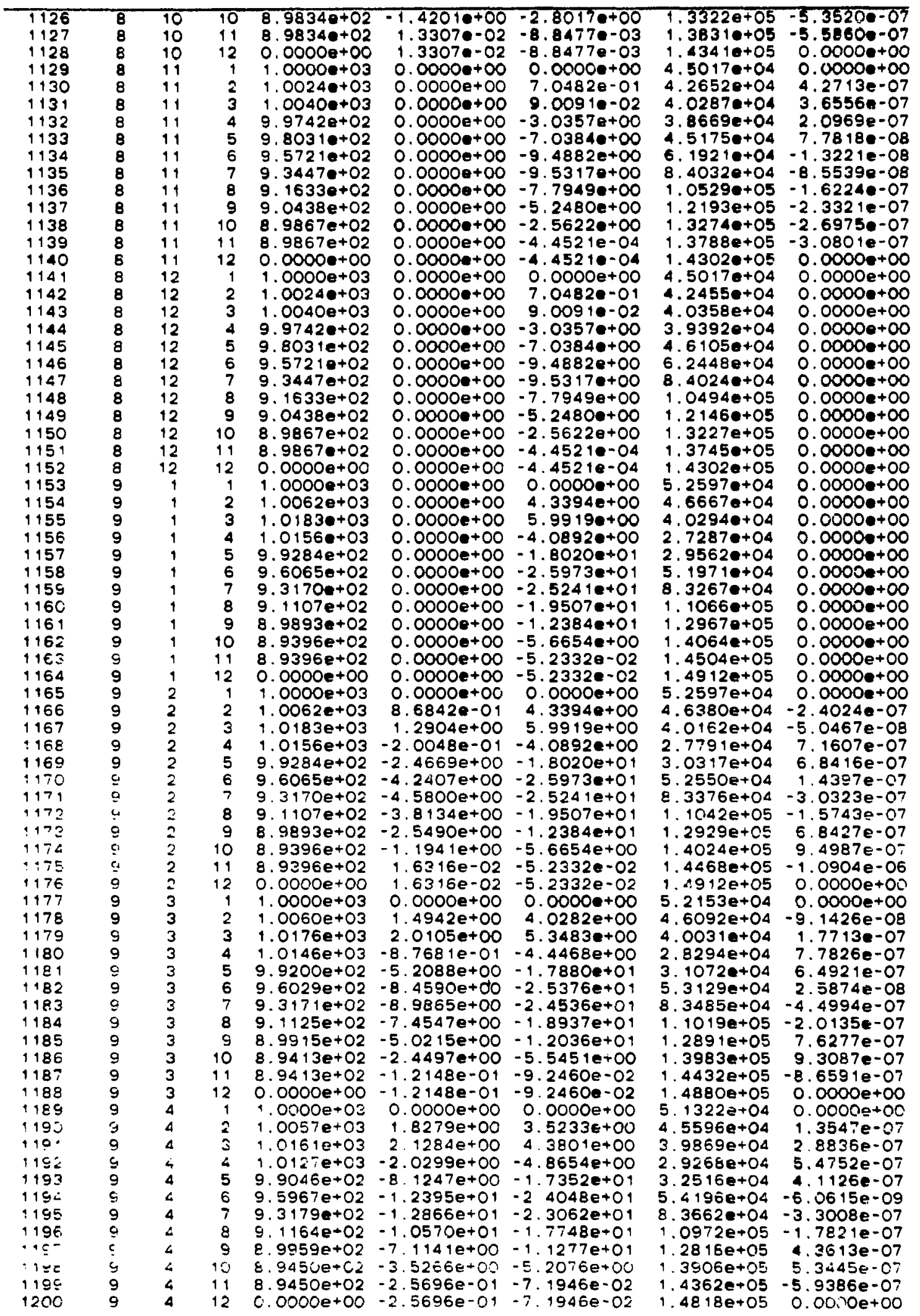


Flow over step - Inflow velocity - 1000. CRAY XMP24 10/30/87 09:05:41 itere 1 time $4.001190-01$

eycle:

2216
$\mathbf{M}$ K J I
$\mathbf{V}$
Q $v$

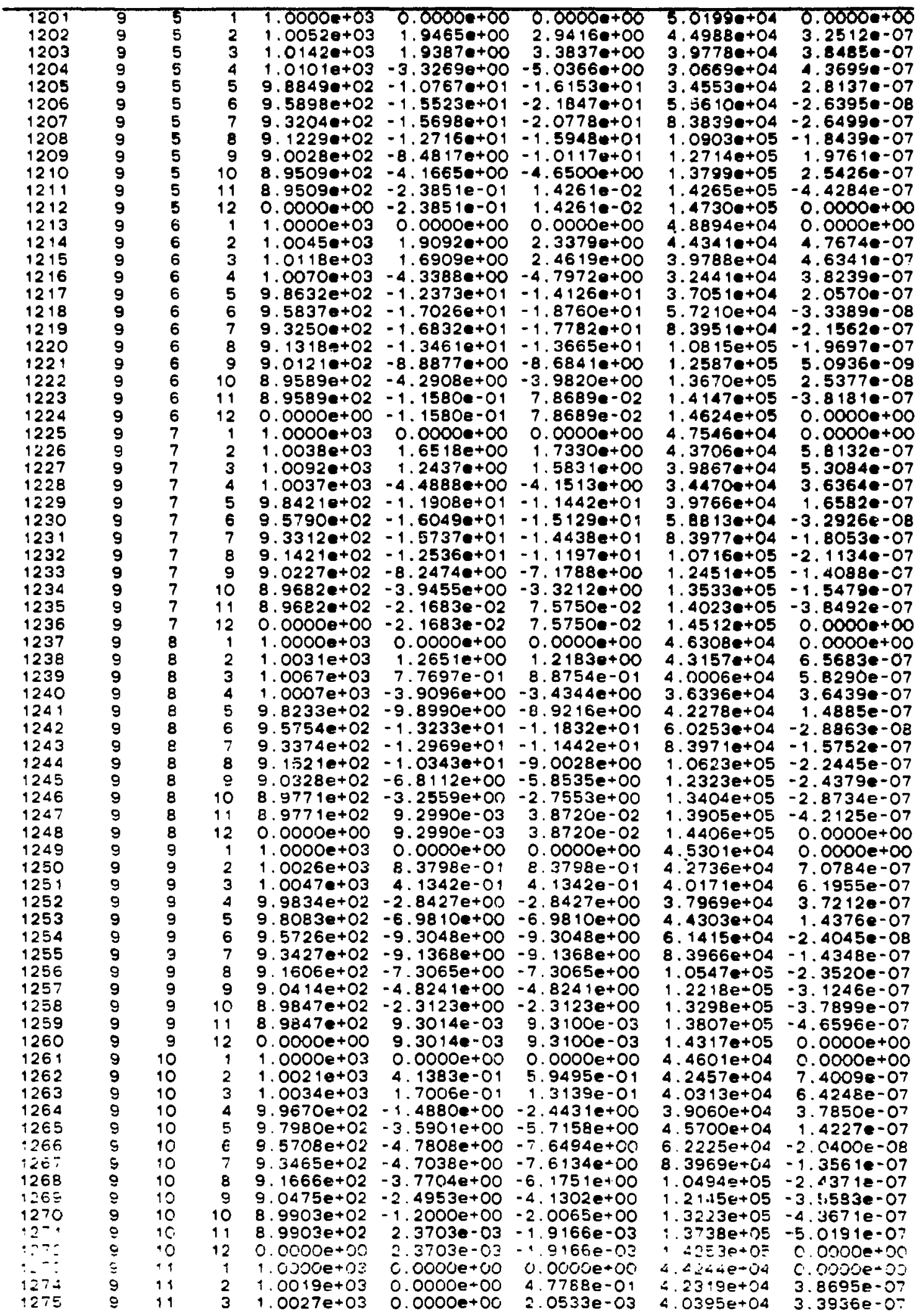


Flow over atep. Inflow velocity - 1000. CRAY XMP24 10/30/87 00:05:44 ttere 1 time=4.001190-01

cyele:

2216

M $K$ J I

$\mathbf{u}$

$\checkmark$

$w$

$p$

div

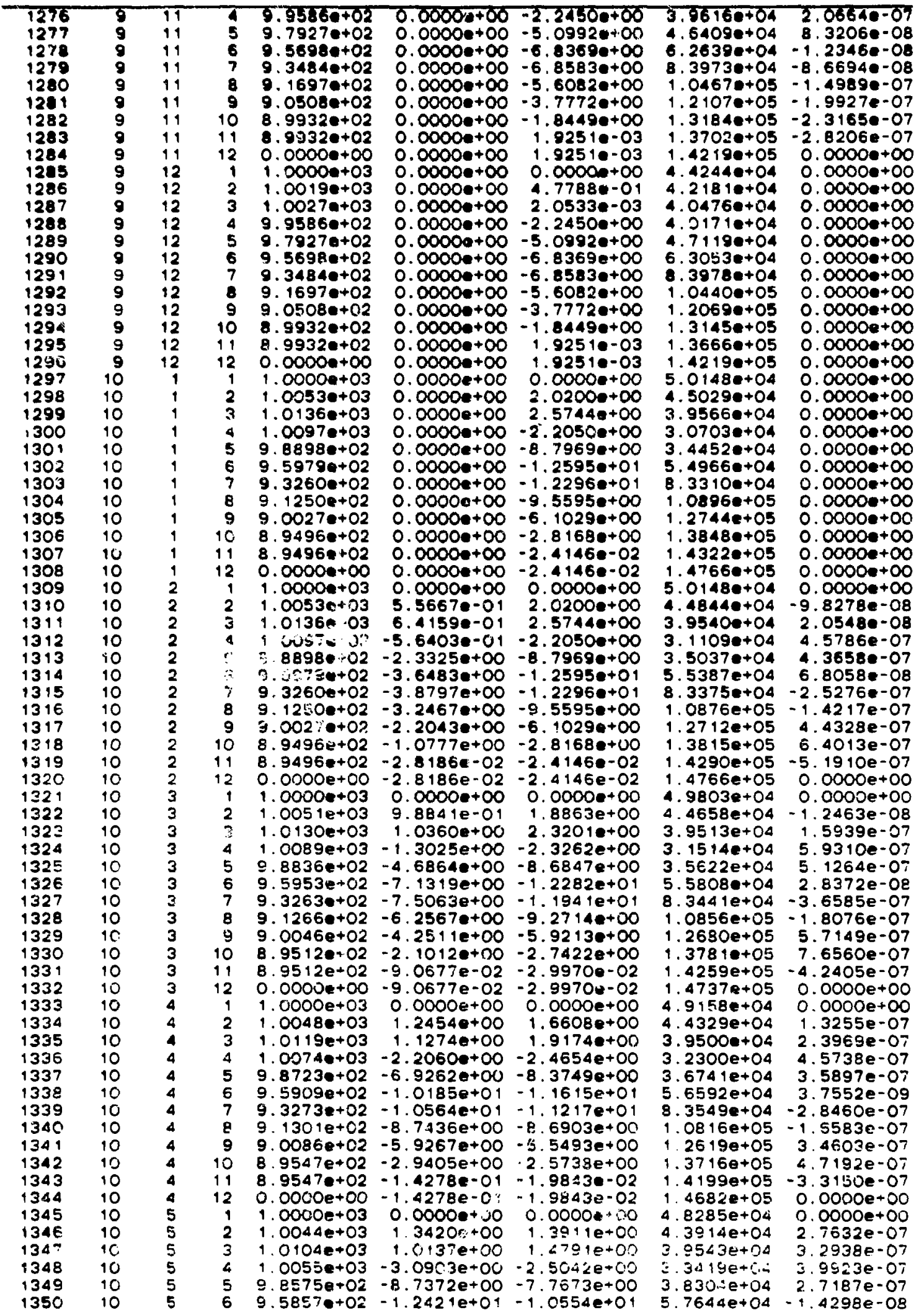


Flow over steo. Inflow velocity * 1000. CRAY XMP24 10/30/87 08:05:41 itere 1 time.4.001190-01

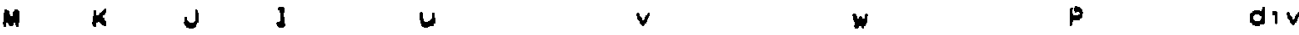

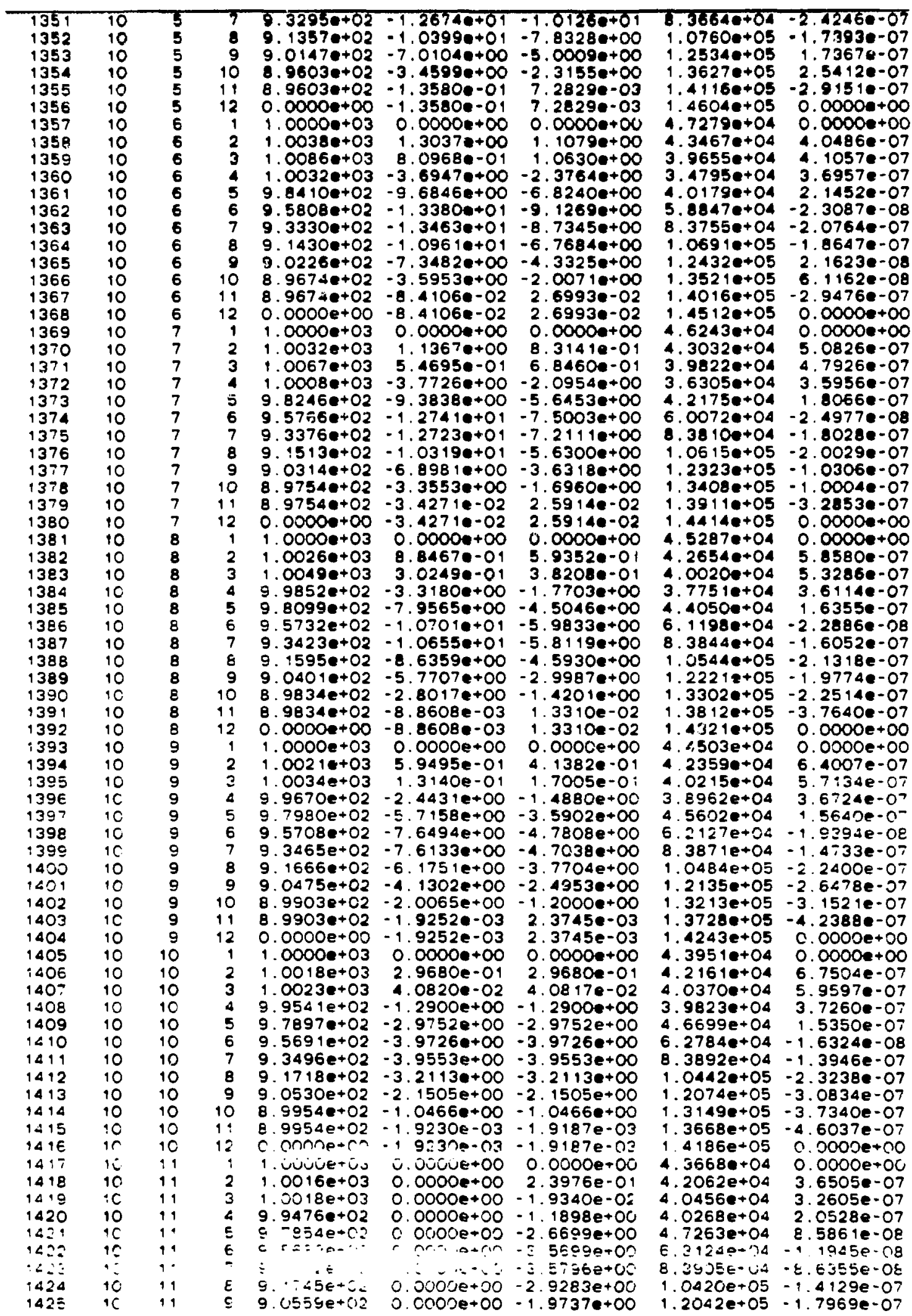


Flow over steo. Inflow velocity. 1000. CRAY XMP24 10/30/87 09:05:41 iter. 1 time=4.001190-01
m K J I
$\mathbf{u}$

$$
\checkmark
$$
div

\begin{tabular}{|c|c|c|c|c|c|c|c|c|}
\hline 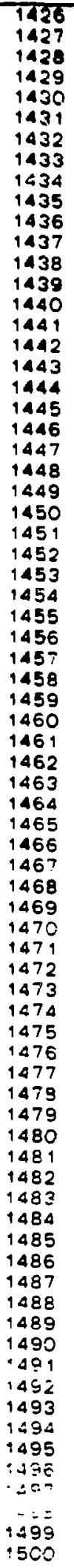 & 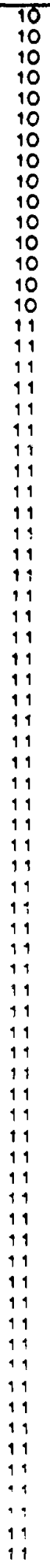 & 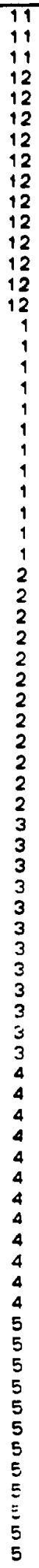 & 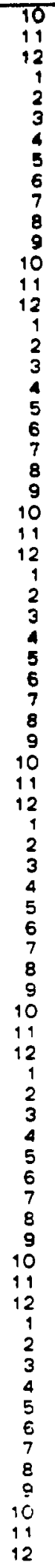 & $\begin{array}{l}1.9981 e+C 2 \\
8.9981 e+02 \\
0.0000+00 \\
1.0000+03 \\
1.0016 e+03 \\
1.0018 e+03 \\
9.9476 e+02 \\
9.7851 e+02 \\
9.5682++02 \\
9.3512 e+02 \\
9.1745 e+02 \\
9.0559 e+02 \\
8.9981 e+02 \\
8.9981 e+02 \\
0.0000 e+00 \\
1.0000 e+03 \\
1.0048 e+03 \\
1.0114 e+03 \\
1.0068 e+03 \\
9.8708 e+02 \\
9.5930 e+02 \\
9.3296 e+02 \\
9.1313 e+02 \\
9.0086 e+02 \\
8.9539 e+02 \\
8.9539 e+02 \\
0.0000 e+00 \\
1.0000 e+03 \\
1.0048 e+03 \\
1.0114 e+03 \\
1.0068 e+03 \\
9.8708 e+02 \\
9.5930 e+02 \\
9.3296 e+02 \\
9.1313 e+02 \\
9.0086 e+02 \\
8.9539 e+02 \\
8.9539 e+02 \\
0.0000 e+00 \\
1.0000 e+03 \\
1.0046 e+03 \\
1.0109 e+03 \\
1.0062 e+03 \\
9.8657 e+02 \\
9.5909 e+02 \\
9.3301 e+02 \\
9.1330 e+02 \\
9.0105 e+02 \\
8.9557 e+02 \\
8.9557 e+02 \\
0.0000 e+00 \\
1.0000 e+03 \\
1.0043 e+03 \\
1.0099 e+03 \\
1.0049 e+03 \\
9.8563 e+02 \\
9.5874 e+02 \\
9.3312 e+02 \\
9.1364 e+02 \\
9.0145 e+02 \\
8.9593 e+02 \\
8.9593 e+02 \\
0.0000 e+00 \\
1.0000 e+03 \\
1.0039 e+03 \\
1.0086 e+03 \\
1.0033 e+03 \\
9.8439 e+02 \\
9.5831 e+02 \\
9.3334 e+02 \\
9.1416 e+02 \\
9.0203 e+02 \\
8.964 i e+02 \\
8.9647 e+02 \\
0.0000 e+00\end{array}$ & 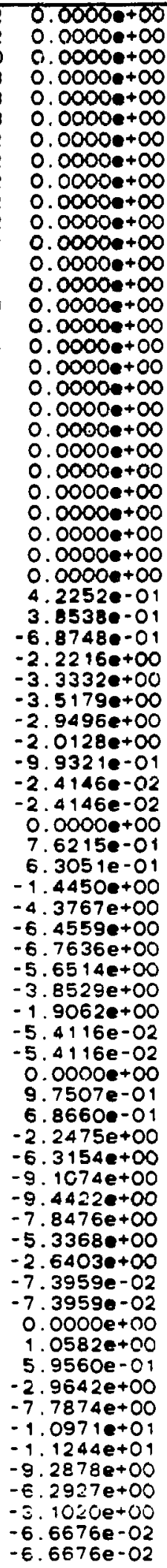 & 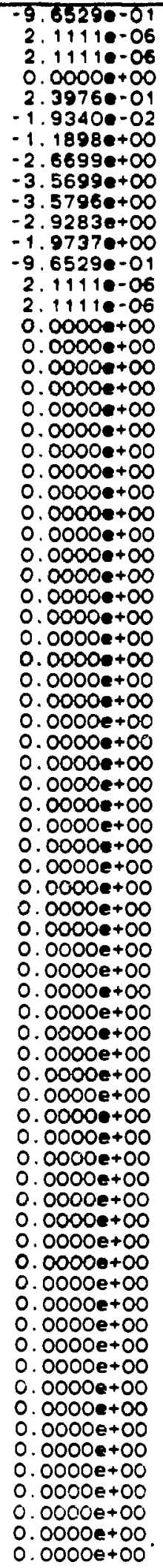 & $\begin{array}{l}1.3146 e+05 \\
1.3636 e+05 \\
1.4157 e+05 \\
4.3668 e+04 \\
4.1963 e+04 \\
4.0541 e+04 \\
4.0713 e+04 \\
4.7827 e+04 \\
6.3464 e+04 \\
8.3917 e+04 \\
1.0398 e+05 \\
1.2010 e+05 \\
1.3083 e+05 \\
1.3605 e+05 \\
1.4157 e+05 \\
4.8887 e+04 \\
4.4217 e+04 \\
3.9251 e+04 \\
3.2265 e+04 \\
3.6705 e+04 \\
5.6331 e+04 \\
8.3288 e+04 \\
1.0809 e+05 \\
1.2630 e+05 \\
1.373 e e+05 \\
1.4226 e+05 \\
1.4687 e+05 \\
4.8887 e+04 \\
4.1075 e+04 \\
3.9264 e+04 \\
3.2625 e+04 \\
3.7210 e+04 \\
5.6681 e+04 \\
8.3335 e+04 \\
1.0791 e+05 \\
1.2602 e+05 \\
1.3707 e+05 \\
1.4197 e+05 \\
1.4687 e+05 \\
4.8591 e+04 \\
4.3934 e+04 \\
3.9277 e+04 \\
3.2986 e+04 \\
3.7715 e+04 \\
5.7031 e+04 \\
8.3382 e+04 \\
1.0772 e+05 \\
1.2573 e+05 \\
1.3677 e+05 \\
1.4168 e+05 \\
1.4660 e+05 \\
4.8038 e+04 \\
4.3679 e+04 \\
3.9321 e+04 \\
3.3682 e+04 \\
3.8679 e+04 \\
5.7688 e+04 \\
8.3461 e+04 \\
1.0737 e++05 \\
1.2518 e+05 \\
1.3618 e+05 \\
1.4113 e+05 \\
1.4608 e+05 \\
4.7289 e+04 \\
4.3354 e+04 \\
3.9418 e+04 \\
3.4665 e+04 \\
4.3019 e+04 \\
5.8575 e+04 \\
8.3549 e+04 \\
1.0687 e+05 \\
1.2442 e+05 \\
1.3537 e+05 \\
1.4036 e+05 \\
1.4535 e+05\end{array}$ & 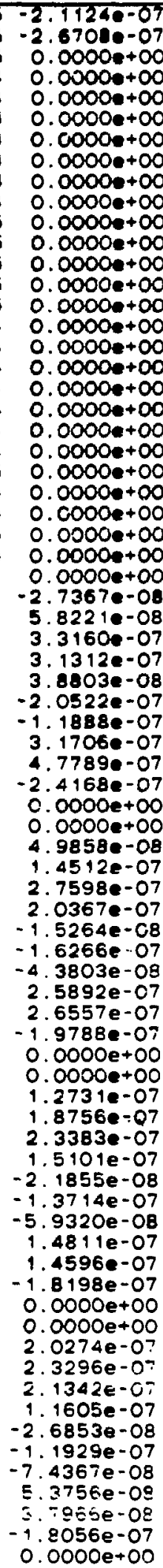 \\
\hline
\end{tabular}


Flow over step - Inflow veloc:ty 1000 . CRAY XMP24 10/30/87 09:05:41 iter= 1 t1me.4.00119.01

M

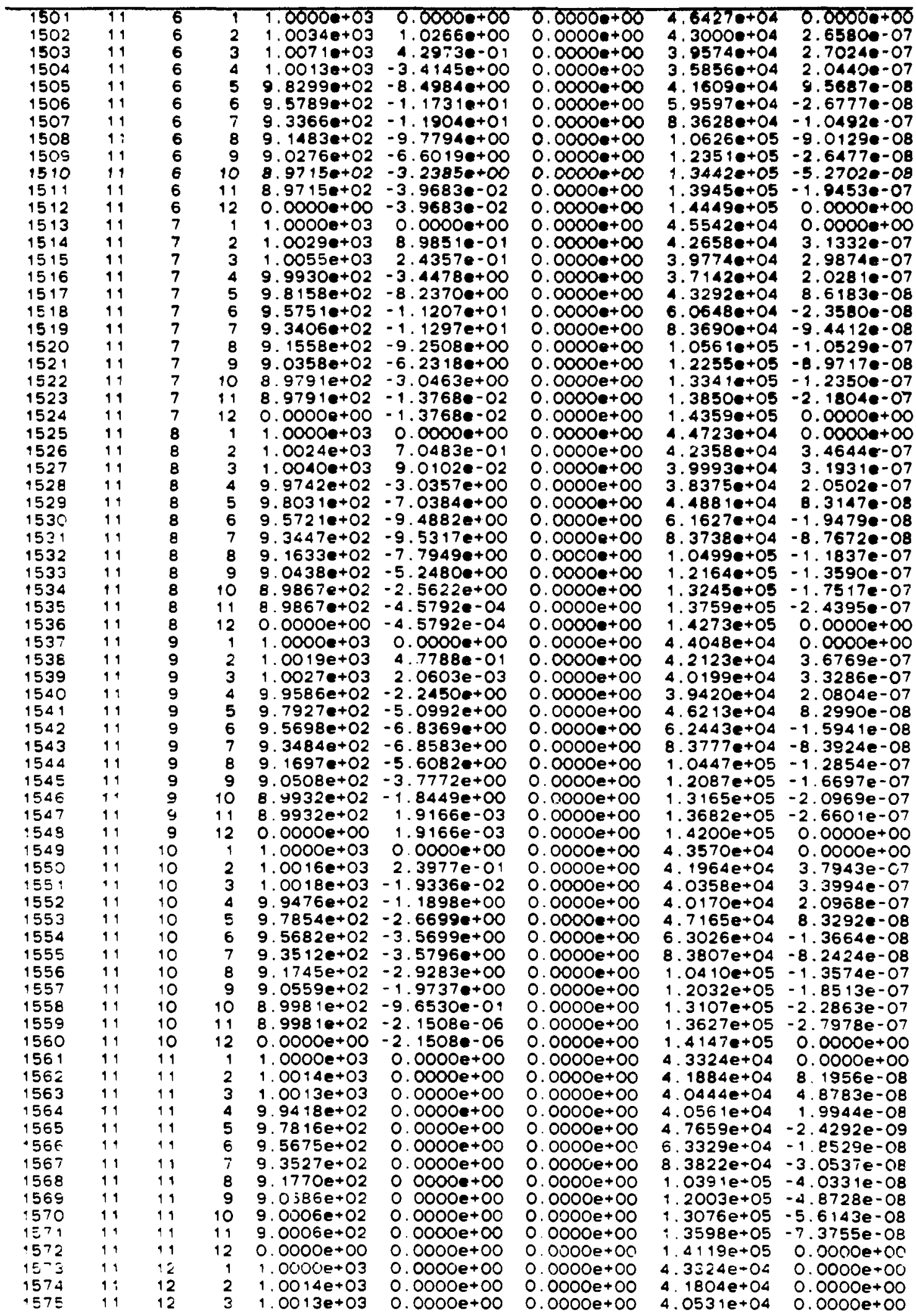


$00+20000 \cdot 0$ $00+80000 \cdot 0$ $00+30000 \cdot 0$ $00+20000 \cdot 0$ $00+20000.0$ $00+\infty 0000 \cdot 0$ $00+20000 \cdot 0$ $00+20000 \cdot 0$ $20+300000$ $00+20000 \cdot 0$ $00+20000 \cdot 0$ $00+20000 \cdot 0$ $00+20000 \cdot 0$ $00+\infty 0000 \cdot 0$ $00+20000 \cdot 0$ $00+\infty 0000 \cdot 0$ $00+\infty 0000 \cdot 0$ $00+\infty 0000^{\circ} 0$ $00+80000 \cdot 0$ $00+\infty 0000 \cdot 0$ $00+20000 \cdot 0$ $00+\infty 0000 \cdot 0$ $00+\infty 0000 \cdot 0$ $00+20000 \cdot 0$ $00 \cdot \otimes 0000 \cdot 0$ $00+20000 \cdot 0$ $00+00000 \cdot 0$ $00+20000 \cdot 0$ $00+20000 \cdot 0$ $00+20000 \cdot 0$ $00+\infty 0000 \cdot 0$ $00+20000 \cdot 0$ $00+20000 \cdot 0$ $00+20000 \cdot 0$ $00+20000^{\prime} 0$ $20+20000$ '0 $00+0000 \cdot 0$ $00+20000^{\circ} 0$ $00+20000 \cdot 0$ $60+20000 \cdot 0$ $00+00000 \cdot 0$ $00++0000 \cdot 0$ $00+00000 \cdot 0$ $00+\infty 0000 \cdot 0$ $00+0000 \cdot 0$ $00+20000 \cdot 0$ $00+\infty 0000$ ' 0 $00+\infty 0000 \cdot 0$ $00+\infty 0000 \cdot 0$ $00+\infty 0000 \cdot 0$ $00+0000 \cdot 0$ $00+00000 \cdot 0$ $00+20000 \cdot 0$ $00+\infty 0000 \cdot 0$ $00+\infty 0000 \cdot 0$ $00+00000 \cdot 0$ $00+\infty 0000 \cdot 0$ $00+\infty 0000 \cdot 0$ $00+\infty 0000 \cdot 0$ $00+\infty 0000 \cdot 0$ $00+\infty 0000^{\circ} 0$ $00+\infty 0000 \cdot 0$ $00+\infty 0000$ '0 $00+00000$ 'O $00+\infty 0000$ '0 $00+\infty 0000$ '0 $00+\infty 0000 \cdot 0$ $00+\infty 0000$ '0 $00+\infty 0000 \cdot 0$ $00+\infty 0000 \cdot 0$ $00+00000 \cdot 0$ $00+\infty 0000 \cdot 0$ $00+00000$ '0 $00+\infty 0000 \cdot 0$ $00+\infty 0000 \cdot 0$ $\nabla 0+a \angle \nabla \varepsilon O \cdot 9$ $\triangle O+20 \nabla O E$. $00+\partial \leq 169$. $\nabla 0+a \varepsilon 6 \nabla \sigma^{\prime} E$ $\nabla 0+\partial \nabla \varepsilon \varsigma 2$ $00+\theta<\tau \nabla 9^{\circ}$ SO+OSESD ' SO+ $\angle$ LSEE । SO+อ8 $\nabla \nabla \varepsilon \cdot 1$ SO+ $06 \nabla \varepsilon \tau$. $90+\theta \varepsilon, 90 \cdot b$

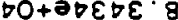
$\checkmark O+2$ LOS6. $S$ $\nabla O+\theta \varepsilon E L 1$. $\forall 0+2016 S^{\circ} \varepsilon$ $\nabla 0+\partial \nabla 626^{\circ} \varepsilon$ $D O+\theta E G \angle Z^{\circ}$ $\triangle 0+268 Z L^{\circ} \nabla$ SO+OBC9० SO+a LZO ' ' SO+०OZGE. SO+0 LIDZ SO+28S90 ' $\neg O+\partial \tau L E E \cdot \theta$ $-0+a b 8<\theta^{\circ} \cdot 9$ $>0+\theta \leq 190 \circ$ $\circ 0+a \varepsilon 909^{\circ} \varepsilon$ $\checkmark 0+a z \downarrow \mid 6^{\circ} \varepsilon$

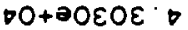
$\triangle 0+08 \varepsilon 00^{\circ}$ SO+2099 ' SO+ 28 LOV SO+0ट $\angle S E$. SO+099 02 . $90+06890^{\circ}$ $\succ 0+\bullet \varepsilon 乙 \varepsilon \varepsilon \cdot 8$ $60+0+528 \cdot 9$ $70+0 \angle 086^{\circ} \varepsilon$ $\checkmark O+\theta \angle S D D \cdot \varepsilon$ $\nabla 0+\infty 0 \nabla 06^{\circ} \varepsilon$ $\checkmark 0+\infty 012 E^{\circ}$ $00+01698$. CO+C LB9 SO+* + OL $\square$ $50+0009 \varepsilon^{\prime \prime}$

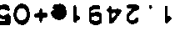
$90+90<0^{\circ}$ $0+0$ S $2 E \cdot B$

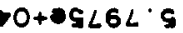
$0+028 \varepsilon 6$ $10+0 z b 1 \cdot \varepsilon$ $0+0886 B \cdot \varepsilon$ DO+ LOEE " $\checkmark 0+\angle 88^{\circ}$. $90+0<80^{\circ}$ co+egrट.

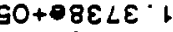
$0+00 \varepsilon 92$. $50+66080$. $0+0882 \varepsilon \cdot 8$ $0+0$ เ $\varepsilon \varepsilon 9 \cdot \mathbf{s}$ O+०SOL9 E $0+0592 z^{\circ} \varepsilon$ ००+०เgZ6. $0+0<120^{\circ}$ $0+\circ<888^{\circ} \circ$ $90+06116$ SO+069SE. SO+ $9 \nabla 0 \varepsilon$. $50+0$ S $\angle 6$. SO+QZ LEO $-0+08 \varepsilon 8 \varepsilon^{\prime} 8$

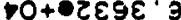
$10+0 \varepsilon 510$. $10+02560^{\circ}$
$00+70000 \cdot 0$ $00+20000^{\prime} 0$ $00+25000^{\prime} 0$ $00+30000 \cdot 0$ $00+\theta 0000 \cdot 0$ $00+20000 \cdot 0$ $130+20000^{\circ} 0$ $\mathrm{jO}+20000 \cdot 0$ $00+20000 \cdot 5$ $00+20000 \cdot 0$ $00+00000^{\circ} 0$ $00+20000^{\circ} 0$ $00+20000 \cdot 0$ $00+20000^{\circ} 0$ $00+00000^{\circ} 0$ $00+20000^{\circ} 0$ $00+80000^{\circ} 0$ $00+20000 \cdot 0$ $00+20000^{\circ} 0$ $00+80000 \cdot 0$ $00+20000 \cdot 0$ $00+00000 \cdot 0$ $00+20000 \cdot 0$ $00+20000^{\prime} 0$ $00+20000^{\circ} 0$ $00+20000 \cdot 0$ $00+20000 \cdot 0$ $00+20000 \cdot 0$ $00+70000$ 'O $00+20000 \cdot 0$ $00+00000 \cdot 0$ $00+20000 \cdot 0$ $00++0000^{\prime} 0$ $00+20000 \cdot 0$ $00+\infty 0000 \cdot 0$ $00+80000 \cdot 0$ $00+\infty 0000 \cdot 0$ $00+20000^{\circ} 0$ $00+\infty 0000 \cdot 0$ $00+\infty 0000^{\circ} 0$ $00+20000 \cdot 0$ $00+\infty 0000^{\circ} 0$ $00+00000^{\circ} 0$ $00+0000 \cdot 0$ $00+\infty 0000 \cdot 0$ $00+80000 \cdot 0$ $00+\infty 0000^{\circ} 0$ $00+80000 \cdot 0$ $00+\infty 0000 \cdot 0$ $00+\infty 0000^{\circ} 0$ $00+20000 \cdot 0$ $00+\infty 0000 \cdot 0$ $00+80000 \cdot 0$ $00+\infty 0000 \cdot 0$ $00+\infty 0000 \cdot 0$ $00+\infty 0000^{\circ} 0$ $00+\infty 0000 \cdot 0$ $00+\infty 0000 \cdot 0$ $00+\infty 0000 \cdot 0$ $00+00000 \cdot 0$ $00+00000^{\circ} 0$ $00+20000 \cdot 0$ $00+90000 \cdot 0$ $00+00000 \cdot 0$ $00+\infty 0000 \cdot 0$ $00+\infty 0000^{\circ}$ $00+\infty 0000 \cdot 0$ $00++0000 \cdot 0$ $00+\infty 0000 \cdot 0$ $00+00000 \cdot 0$ $00+20000 \cdot 0$ $00+\infty 0000 \cdot 0$ $00+90000^{\circ} 0$ $00+\infty 0000^{\circ}$ $00+\infty 0000 \cdot 0$

bO+a LELb $b-20+368 \angle G \cdot 6$ $00+2 \nabla 86 \downarrow \cdot 8-i 0+26628 \cdot 6$

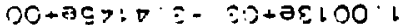

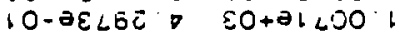
$00+29 Z^{\prime} O^{\prime} \quad \varepsilon 0+\theta 0 \varepsilon 00^{\circ}$ $00+20000$ o $\varepsilon 0+20000$ : b $20-9199 \cdot 9-00+00000 \cdot 0$ $20-39 \angle 99 \cdot 9-20+2 \angle b 96 \cdot 8$ $30+00201 \cdot \varepsilon-20+\angle 096 \cdot 8$ $00+2 L 26 \geqslant \cdot 9-20+\theta \varepsilon 020 \cdot 6$ $00+\otimes B<82 \cdot 6-20+29101 \cdot 6$

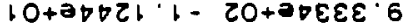
$10+01 \angle 60^{\circ} 1-20+2 ! E 8 S^{\circ} 6$ $00+\theta \nabla \angle 8 L \cdot L-20+\theta 6 \varepsilon \nabla 8 \cdot 6$ $00+\theta \tau b 96^{\circ} \tau-\varepsilon 0+\theta \varepsilon \varepsilon 00^{\prime} b$ $10-00956^{\circ} \mathrm{g} \quad \varepsilon 0+09800 \%$ $00+22850$. $\varepsilon 0+26 \varepsilon 00 \%$ $00+20000^{\circ} \mathrm{O} \quad \varepsilon 0+20000^{\circ}$. $20-2656 \varepsilon \cdot L-00+20000^{\circ} 0$ ZO-จ6S6E $L-20+\theta \varepsilon 696 \cdot 8$ $00+\partial \varepsilon 0 \times 9 \cdot \tau-\tau 0+\cos 66^{\prime} \cdot$ $00+089 \varepsilon E \cdot s-20+25010 \cdot 6$ $00+a 9 \angle b 8^{\circ} L-20+0 b 9 E L^{\prime} \cdot 6$

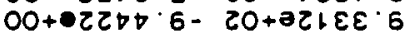
$0 O+a \nabla \angle O F \cdot 6-20+2 b \angle B S \cdot 6$ OO+OЬSเE' 9 - $20+\partial \varepsilon 9 S 8 \cdot 6$ $00+\theta g \angle \nabla \tau \cdot \tau-\varepsilon 0+06 \nabla 00 \%$ b0-00998'9 EO+26600.

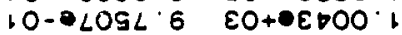
$00+20000 \cdot 0 \quad E 0+20000 \%$ 20-0gl b ' s- $00+\infty 0000^{\circ} 0$

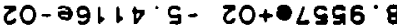
$00+\theta 2906^{\prime} b-20+0$ LSS6 $^{\circ} 8$ $00+26258 \cdot \varepsilon-20+05010 \cdot 6$ $00+001.2 \cdot \mathrm{s}-20+00 \varepsilon \varepsilon \mathrm{r} \cdot 6$ OO+89E9L'9- ZO+OLOEE 6 $00+2695 V \cdot 9-20+06069 \cdot 6$ $00+0<9 \angle \varepsilon \cdot \bullet-20+0 \angle 998 \cdot 6$ OO+ OOSO : - EO+e2900. lO-OLSOE'9 $\varepsilon 0+06010$. เO-2S।Z9.' $\quad \varepsilon 0+99+00$. $00+\infty 0000 \cdot 0 \quad \varepsilon 0+20000 \%$ $20-0901+\tau \cdot 00+\infty 0000 \cdot 0$ 20-9916. $2-20+26 \varepsilon 56 \cdot 8$ $10-12 \varepsilon 6^{-6}-20+06 E \xi 6 \cdot 8$ $00+\theta 8210^{\circ} \tau-20+89800^{\prime} 6$ $00+9676 \cdot \tau-20+\theta \varepsilon+\varepsilon b \cdot 6$ $00+06 L 1 G^{\prime} \varepsilon-\tau 0+096 \tau \varepsilon \cdot 6$ $\infty+2 \tau \varepsilon \varepsilon \varepsilon \cdot \varepsilon-20+00 \varepsilon 6 \mathrm{~S} \cdot 6$ $00+09122 \cdot 2 \cdot 20+e 80 \angle 8 \cdot 6$ $10-80<8 \cdot 9-\varepsilon 0+08900$ । |Olo-erszr' $\varepsilon 0+98000$. $00+\infty 0000 \cdot 0 \quad \varepsilon 0+\infty 0000$. $00+00000$ 'O $00+20000$. $00+\infty 0000^{\circ} 0 \quad 20+06 \varepsilon 56$. $00+\infty 0000 \cdot 0$ $00+\infty 0000 \cdot 0$ $00+\infty 0000 \cdot 0$ $00+00000 \cdot 0$ $00+\infty 0000 \cdot 0$ $00+\infty 0000 \cdot 0$ $00+00000 \cdot 0$ $00+\infty 0000 \cdot 0$ $00+\infty 0000 \cdot 0$ $00+\infty 0000 \cdot 0$ 
Flow over step Inflow veloctty. 1000 * CRAY XMP24 10/30/87 09:05:41, titera, 1 time" 4.001190-01

cyclen 2216 K J I $u$ $v$
div

\begin{tabular}{|c|c|c|c|c|c|c|c|c|}
\hline $\begin{array}{l}1651 \\
1652 \\
1653 \\
1654 \\
1655 \\
1656 \\
1657 \\
1658 \\
1659 \\
1660 \\
1661 \\
1662 \\
1663 \\
1664 \\
1665 \\
1666 \\
1667 \\
1668 \\
1669 \\
1670 \\
1671 \\
1672 \\
1673 \\
1674 \\
1675 \\
1676 \\
1677 \\
1678 \\
1679 \\
1680 \\
1681 \\
1682 \\
1683 \\
1684 \\
1685 \\
1686 \\
1687 \\
1688 \\
1689 \\
1690 \\
1691 \\
1692 \\
1693 \\
1694 \\
1695 \\
1696\end{array}$ & $\begin{array}{l}12 \\
12 \\
12 \\
12 \\
12 \\
12 \\
12 \\
12 \\
12 \\
12 \\
12 \\
12 \\
12 \\
12 \\
12 \\
12 \\
12 \\
12 \\
12 \\
12 \\
12 \\
12 \\
12 \\
12 \\
12 \\
12 \\
12 \\
12 \\
12 \\
12 \\
12 \\
12 \\
12 \\
12 \\
12 \\
12 \\
12 \\
12 \\
12 \\
12 \\
12 \\
12 \\
12 \\
12 \\
12 \\
12 \\
12 \\
12 \\
12 \\
12 \\
12 \\
12 \\
12 \\
12 \\
12 \\
12 \\
12 \\
12 \\
12 \\
12 \\
12 \\
12 \\
12 \\
12 \\
12 \\
12\end{array}$ & 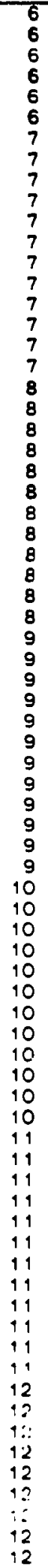 & 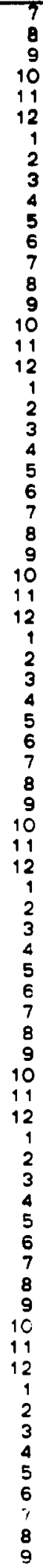 & $\begin{array}{l}9.3366 e+02 \\
9.1483 e+02 \\
9.0276 e+02 \\
8.9715 e+02 \\
8.9715 e+02 \\
0.0000 e+00 \\
1.0000 e+03 \\
1.0029 e+03 \\
1.0055 e+03 \\
9.9930 e+02 \\
9.8158 e+02 \\
9.5751 e+02 \\
9.3406 e+02 \\
9.1558 e+02 \\
9.0358 e+02 \\
8.9791 e+02 \\
8.9791 e+02 \\
0.0000 e+00 \\
1.0000 e+03 \\
1.0024 e+03 \\
1.0040 e+03 \\
9.9742 e+02 \\
9.8031 e+02 \\
9.5721 e+02 \\
9.3447 e+02 \\
9.1633 e+02 \\
9.0438 e+02 \\
8.9867 e+02 \\
8.9867 e+02 \\
0.0000 e+00 \\
1.0000 e+03 \\
1.0019 e+03 \\
1.0027 e+03 \\
9.9586 e+02 \\
9.7927 e+02 \\
9.5698 e+02 \\
9.3484 e+02 \\
9.1697 .+02 \\
9.0508 e+02 \\
8.9932 e+02 \\
8.9932 e+02 \\
0.0000 e+00 \\
1.0000 e+03 \\
1.0016 e+03 \\
1.0018 e+03 \\
9.9476 e+02 \\
9.7854 e+02 \\
9.5682 e+02 \\
9.3512 e+02 \\
9.1745 e+02 \\
9.0559 e+02 \\
8.9981 e+02 \\
8.9981 e+02 \\
0.0000 e+00 \\
1.0000 e+03 \\
1.0014 e+03 \\
1.0013 e+03 \\
9.9418 e+02 \\
9.7816 e+02 \\
9.5675 e+02 \\
9.3527 e+02 \\
9.1770 e+02 \\
9.0586 e+02 \\
9.0006 e+02 \\
9.0006 e+02 \\
0.0000 e+00 \\
1.0000 e+03 \\
1.0014 e+03 \\
1.0013 e+03 \\
9.9418 e+02 \\
9.7816 e+02 \\
0.56-5 e+02 \\
9.35 z: e+0=3 \\
9.1770 e+02 \\
9.0586 e+02\end{array}$ & $\begin{array}{r}-1.1904 e+01 \\
-9.7794 e+00 \\
-6.6019 e+00 \\
-3.2385 e+00 \\
-3.9683 e-02 \\
-3.9683 e-02 \\
0.0000 e+00 \\
8.9851 e-01 \\
2.4357 e-01 \\
-3.4478 e+00 \\
-8.2370 e+00 \\
-1.1207 e+01 \\
-1.1297 e+01 \\
-9.2508 e+00 \\
-6.2318 e+00 \\
-3.0463 e+00 \\
-1.3768 e-02 \\
-1.3768 e-02 \\
0.0000 e+00 \\
7.0483 e-01 \\
9.0102 e-02 \\
-3.0357 e+00 \\
-7.0384 e+00 \\
-9.4882 e+00 \\
-9.5317 e+00 \\
-7.7949 e+00 \\
-5.2480 e+00 \\
-2.5622 e+00 \\
-4.5792 e-04 \\
-4.5792 e-04 \\
0.0000 e+00 \\
4.7788 e-01 \\
2.0603 e-03 \\
-2.2450+00 \\
-5.0992 e+00 \\
-6.8369 e+00 \\
-6.8583 e+00 \\
-5.6082 e+00 \\
-3.7772 e+00 \\
-1.8449 e+00 \\
1.9166 e-03 \\
1.9166 e-03 \\
0.0000 e+00 \\
2.3977 e-01 \\
-1.9336 e-02 \\
-1.1898 e+00 \\
-2.6699 e+00 \\
-3.5699 e+00 \\
-3.5796 e+00 \\
-2.9283 e+00 \\
-1.5737 e+00 \\
-9.6530 e-01 \\
-2.1508 e-06 \\
-2.1508 e-06 \\
0.0000 e+00 \\
0.0000 e+00 \\
0.0000 e+00 \\
0.0000 e+00 \\
0.0000 e+00 \\
0.0000 e+00 \\
0.0000 e+00 \\
0.0000 e+00 \\
0.0000 e+00 \\
0.0000 e+00 \\
0.0000 e+00 \\
0.0000 e+00 \\
0.0000 e+00 \\
0.0000 e+00 \\
0.0000 e+00 \\
0.0000 e+00 \\
0.0000 e+00 \\
0.0000 e+00 \\
0.0000 e+00 \\
0.0000 e+00 \\
0.0000 e+00\end{array}$ & 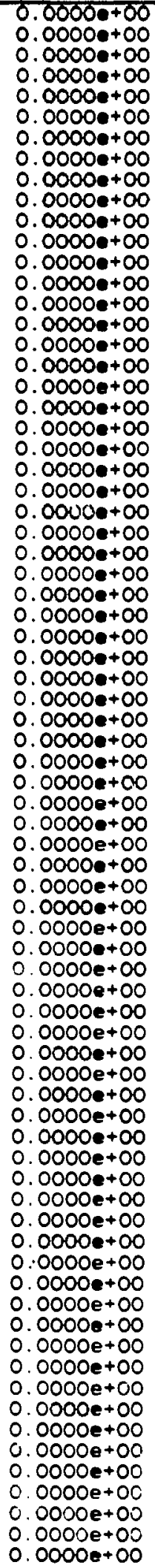 & $\begin{array}{l}8.3501 e+04 \\
1.0561 e+05 \\
1.2269 e+05 \\
1.3362 e+05 \\
1.3875 e+05 \\
1.4449 e+05 \\
4.5542 e+04 \\
4.2283 e+04 \\
3.9726 e+04 \\
3.7979 e+04 \\
4.4408 e+04 \\
6.1225 e+04 \\
8.3570 e+04 \\
1.0507 e+05 \\
1.2186 e+05 \\
1.3273 e+05 \\
1.3788 e+05 \\
1.4359 e+05 \\
4.4723 e+04 \\
4.2063 e+04 \\
3.9966 e+04 \\
3.9000 e+04 \\
4.5713 e+04 \\
6.2056 e+04 \\
8.3632 e+04 \\
1.0455 e+05 \\
1.2107 e+05 \\
1.3188 e+05 \\
1.3706 e+05 \\
1.4273 e+05 \\
4.4048 e+04 \\
4.1887 e+04 \\
4.0182 e+04 \\
3.9877 e+04 \\
4.6825 e+04 \\
6.2759 e+04 \\
8.3684 e+04 \\
1.0411 e+05 \\
1.2039 e+05 \\
1.3116 e+05 \\
1.3636 e+05 \\
1.4200 e+05 \\
4.3570 e+04 \\
4.1767 e+04 \\
4.0345 e+04 \\
4.0517 e+04 \\
4.7631 e+04 \\
6.3268 e+04 \\
8.3721 e+04 \\
1.0379 e+05 \\
1.1991 e+05 \\
1.3064 e+05 \\
1.3585 e+05 \\
1.4147 e+05 \\
4.3324 e+04 \\
4.1706 e+04 \\
4.0433 e+04 \\
4.0854 e+04 \\
4.8055 e+04 \\
6.3534 e+04 \\
8.3740 e+04 \\
1.0362 e+05 \\
1.1965 e+05 \\
1.3036 e+05 \\
1.3559 e++05 \\
1.4119 e+05 \\
4.3324 e+04 \\
4.1804 e+04 \\
4.0531 e+04 \\
4.0952 e+04 \\
4.8153 e+04 \\
6.3632 e+04 \\
8.3838 e+04 \\
1.0372 e+05 \\
1.1975 e+05\end{array}$ & $\begin{array}{l}0 . \\
0 . \\
0 . \\
0 . \\
0 . \\
0 . \\
0 . \\
0 . \\
0 . \\
0 .\end{array}$ \\
\hline
\end{tabular}




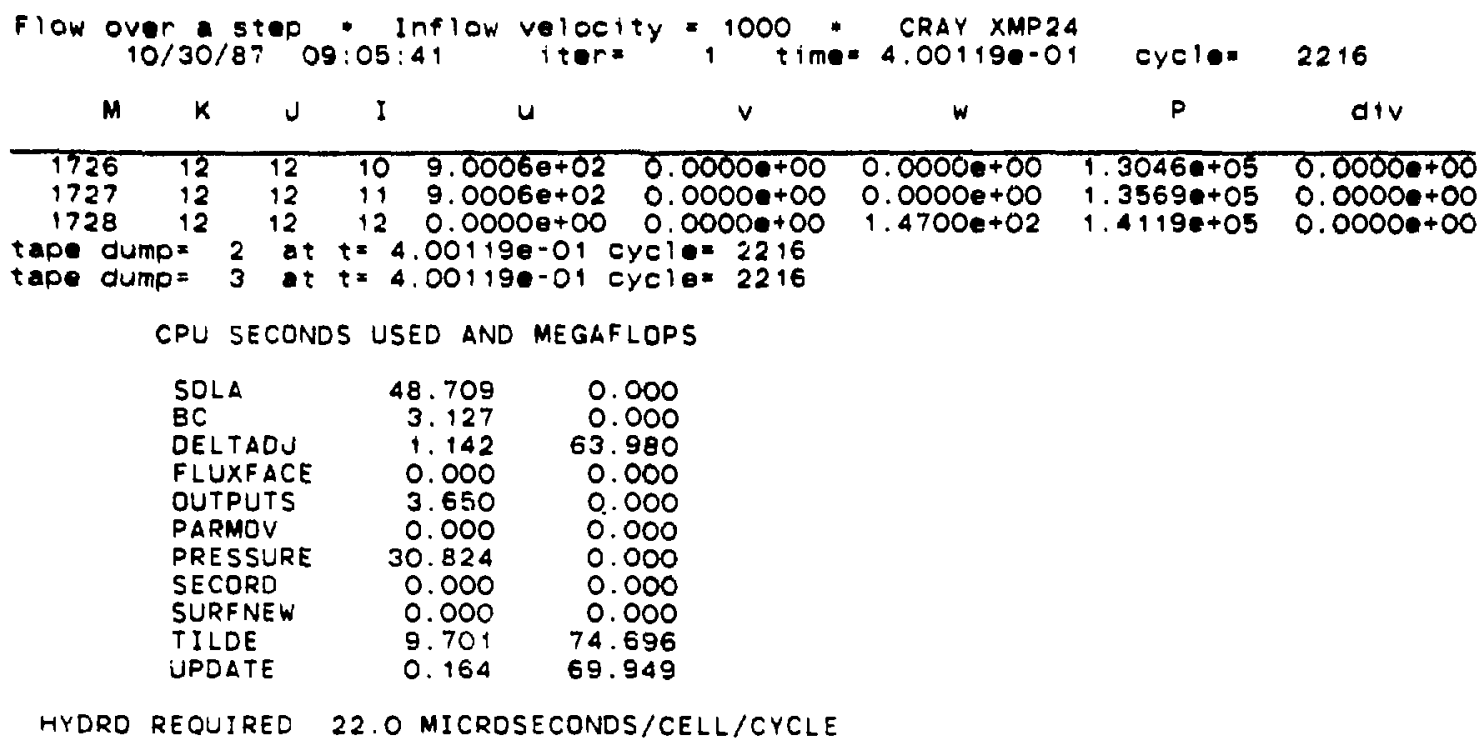

IV. INPUT FILE FOR PROBLEM RESTART

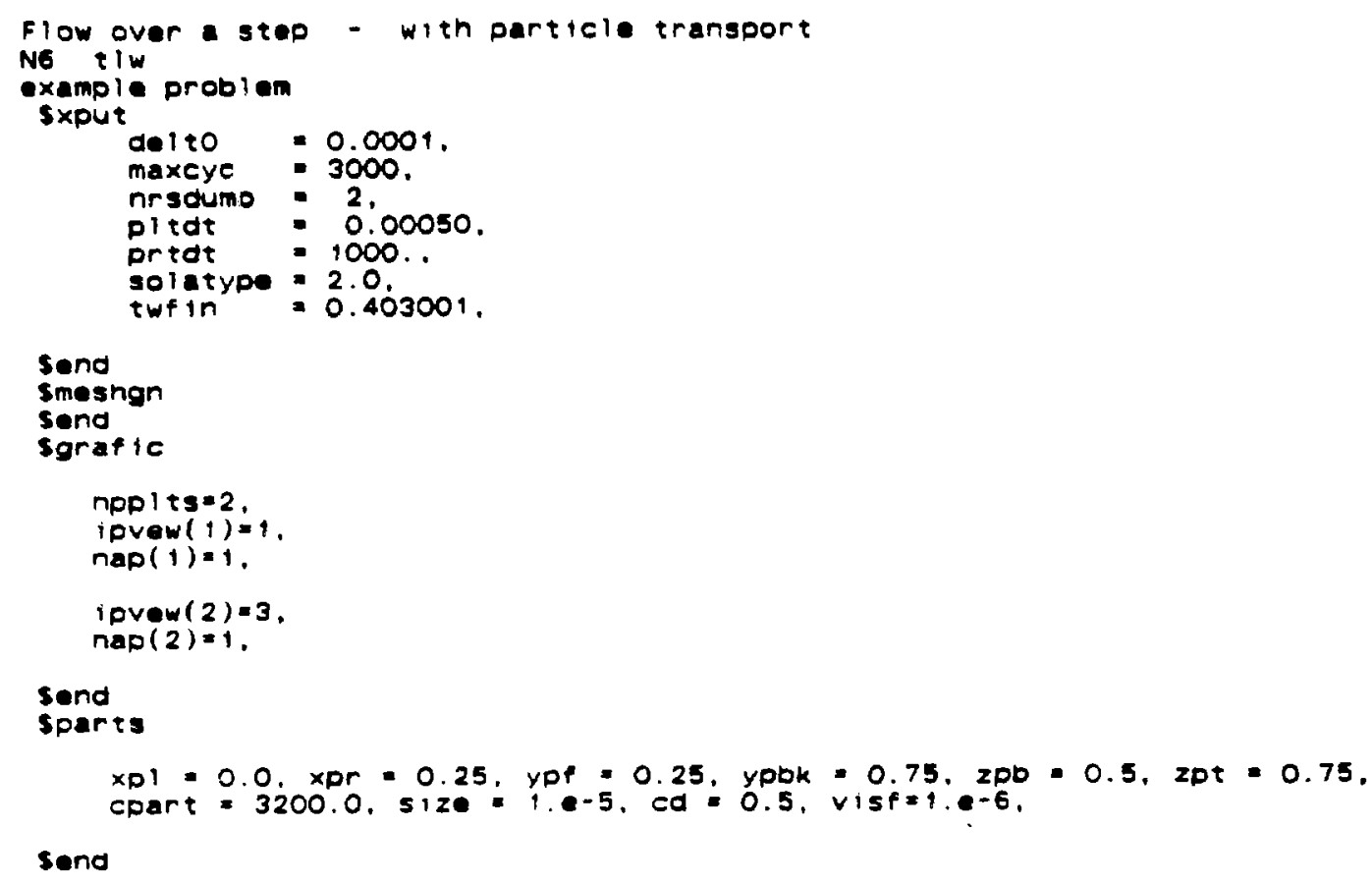

